$$
\text { for }
$$

\title{
Synthesis of Oxygen-Containing Heterocyclic Compounds by Iron-Catalyzed Alkylative Cyclization of Unsaturated Carboxylic Acids and Alcohols
}

\author{
Masayuki Iwasaki, ${ }^{\dagger},{ }^{*}$ Yasuki Kazao, ${ }^{\ddagger}$ Takumi Ishida, ${ }^{\ddagger}$ and Yasushi Nishihara ${ }^{\dagger, *}$ \\ 'Research Institute for Interdisciplinary Science, \\ and ${ }^{*}$ Graduate School of Natural Science and Technology, Okayama University, \\ 3-1-1 Tsushimanaka, Kita-ku, Okayama 700-8530, Japan \\ Phone: +81-86-251-7855 \\ Fax: +81-86-251-7855 \\ E-mail: ynishiha@okayama-u.ac.jp
}

1. General

2. Experimental Procedures and Spectroscopic Data for the New Compounds S3-S14

3. Optimization of Reaction Conditions

4. Substrate Limitation

S20-S21

5.Mechanistic Experiments

S22-S23

6. Copies of ${ }^{1} H,{ }^{13} C\left\{{ }^{1} H\right\}$, and ${ }^{19} F\left\{{ }^{1} H\right\}$ NMR Charts for the New Compounds

S24-S53

7. References 


\section{General}

\section{Instrumentation}

Unless otherwise noted, all the reactions were carried out under an Ar atmosphere using standard Schlenk techniques and heated in a heating block. Solvents were employed as eluents for all other routine operation, as well as dehydrated solvent were purchased from commercial suppliers and employed without any further purification. Glassware was dried in an oven $\left(130^{\circ} \mathrm{C}\right)$ and heated under reduced pressure before use. For thin layer chromatography (TLC) analyses throughout this work, Merck precoated TLC plates (silica gel 60 $\mathrm{GF}_{254}, 0.25 \mathrm{~mm}$ ) were used. Silica gel column chromatography was carried out using Silica gel $60 \mathrm{~N}$ (spherical, neutral, $40-100 \mu \mathrm{m}$ ) from Kanto Chemicals Co., Ltd. NMR spectra $\left({ }^{1} \mathrm{H},{ }^{13} \mathrm{C}\left\{{ }^{1} \mathrm{H}\right\}\right.$, and $\left.{ }^{19} \mathrm{~F}\left\{{ }^{1} \mathrm{H}\right\}\right)$ were recorded on Varian INOVA-600 (600 MHz) or Mercury-400 (400 MHz) spectrometers. Chemical shifts $(\delta)$ are in parts per million relative to $\mathrm{CDCl}_{3}$ at $7.26 \mathrm{ppm}$ for ${ }^{1} \mathrm{H}$ and at $77.16 \mathrm{ppm}$ for ${ }^{13} \mathrm{C}\left\{{ }^{1} \mathrm{H}\right\}$, respectively. The ${ }^{19} \mathrm{~F}\left\{{ }^{1} \mathrm{H}\right\}$ NMR spectra were measured by using $\mathrm{CCl}_{3} \mathrm{~F}(\delta=0.00 \mathrm{ppm})$ as an external standard. The NMR yields were determined by ${ }^{1} \mathrm{H}$ NMR spectra with dibromomethane as an internal standard. Infrared spectra were recorded on a SHIMADZU IRPrestige-21 spectrophotometer. HRMS analyses were obtained by using an ESI-TOF mass spectrometer (Burker micrOTOF) and a double focusing magnetic sector mass spectrometer (JEOL JMS-700 MStation). Elemental analyses were carried out with a Perkin-Elmer $2400 \mathrm{CHN}$ elemental analyzer.

\section{Chemicals}

Unless otherwise noted, materials obtained from commercial suppliers were used without further purification. Nonacarbonyldiiron was purchased from Sigma-Aldrich Co. $N, N, N^{\prime}, N^{\prime}$-Tetramethylurea was purchased from Tokyo Chemical Industry Co., Ltd. Alkenes 1 were prepared according to the literature $^{1}$ and showed the identical spectra reported. Bromides 2 were purchased from commercial suppliers or prepared according to the literature ${ }^{2}$ and showed the identical spectra reported. 


\section{Experimental Procedures and Spectroscopic Data for the New Compounds}

\section{4-Trifluoromethyl-o-vinylbenzoic acid (1d)}<smiles>C=Cc1cc(C(F)(F)F)ccc1C(=O)O</smiles>

1d

White solid obtained by reprecipitation $\left(\mathrm{CH}_{2} \mathrm{Cl}_{2} /\right.$ hexane). Yield was $57 \%$ (329.7 mg). M.p. $128-$ $129^{\circ} \mathrm{C} . \quad$ IR (KBr): 2818 (br), 1694 (s), 1572 (m), 1492 (s) cm ${ }^{-1} . \quad{ }^{1} \mathrm{H}$ NMR (600 MHz, $\left.\mathrm{CDCl}_{3}, \mathrm{rt}\right): \delta 8.15$ $(\mathrm{d}, J=8.4 \mathrm{~Hz}, 1 \mathrm{H}), 7.86(\mathrm{~s}, 1 \mathrm{H}), 7.62(\mathrm{dd}, J=8.4,1.2 \mathrm{~Hz}, 1 \mathrm{H}), 7.57(\mathrm{dd}, J=17.4,11.4 \mathrm{~Hz}, 1 \mathrm{H}), 5.77(\mathrm{dd}, J$ $=17.4,0.6 \mathrm{~Hz}, 1 \mathrm{H}), 5.51(\mathrm{dd}, J=11.4,0.6 \mathrm{~Hz}, 1 \mathrm{H}) ;{ }^{13} \mathrm{C}\left\{{ }^{1} \mathrm{H}\right\} \operatorname{NMR}\left(150 \mathrm{MHz}, \mathrm{CDCl}_{3}, \mathrm{rt}\right): \delta 171.8,141.4$, $134.9,134.8\left(\mathrm{q},{ }^{2} J_{\mathrm{C}-\mathrm{F}}=32.6 \mathrm{~Hz}\right), 132.0,130.2,124.7\left(\mathrm{q},{ }^{3} J_{\mathrm{C}-\mathrm{F}}=3.8 \mathrm{~Hz}\right), 124.2\left(\mathrm{q},{ }^{3} J_{\mathrm{C}-\mathrm{F}}=3.5 \mathrm{~Hz}\right), 123.6(\mathrm{q}$, $\left.{ }^{1} J_{\mathrm{C}-\mathrm{F}}=272 \mathrm{~Hz}\right), 118.9 ;{ }^{19} \mathrm{~F}\left\{{ }^{1} \mathrm{H}\right\}$ NMR (564 MHz, $\left.\mathrm{CDCl}_{3}, \mathrm{rt}\right): \delta-63.4$. Calcd for $\mathrm{C}_{10} \mathrm{H}_{7} \mathrm{~F}_{3} \mathrm{O}_{2}: \mathrm{C}, 55.57 ; \mathrm{H}$, 3.26. Found: C, 55.74; H, 3.07.

A Typical Procedure for Iron-Catalyzed Alkylative Cyclization: Synthesis of 3-(2-Ethoxycarbonyl-2methylpropyl)phthalide (3aa)<smiles>C=Cc1ccccc1C(=O)O</smiles>

$1 \mathrm{a}$<smiles>CCOC(C)(C)Br</smiles>

$2 a$

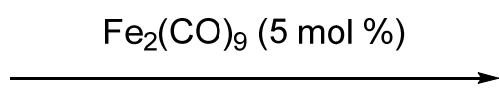

TMU, $80^{\circ} \mathrm{C}, 16 \mathrm{~h}$ $71 \%$

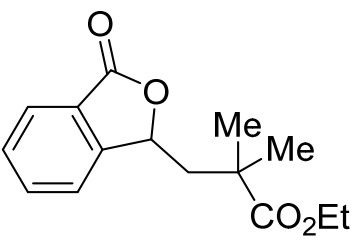

3aa

(6 mmol)

( 2 equiv)

Under an argon atmosphere, nonacarbonyldiiron (109 mg, $0.3 \mathrm{mmol})$ was placed in a 50-mL Schlenk tube. $N, N, N^{\prime}, N^{\prime}$-Tetramethylurea (TMU, $15 \mathrm{~mL}$ ) was then added. After the mixture was stirred for $5 \mathrm{~min}, o-$ vinylbenzoic acid (1a, $889 \mathrm{mg}, 6 \mathrm{mmol}$ ) and ethyl $\alpha$-bromoisobutylate (2a, $2.34 \mathrm{~g}, 12 \mathrm{mmol}$ ) were added to the mixture at room temperature. The mixture was stirred at $80^{\circ} \mathrm{C}$ for $16 \mathrm{~h}$. After the mixture was cooled to room temperature, water $(100 \mathrm{~mL})$ was added to the mixture. The mixture was extracted with ethyl acetate $(30 \mathrm{~mL})$ three times. The combined organic layers were washed with brine $(100 \mathrm{~mL})$ and dried over anhydrous sodium sulfate. After the volatiles were evaporated, the resulting residue was purified by silica gel column chromatography (hexane/ethyl acetate $=3 / 1)$ to provide 3aa as a colorless oil $(1.116 \mathrm{~g}, 4.25 \mathrm{mmol}$, 71\%). $\quad R_{\mathrm{f}}=0.34($ hexane/ethyl acetate $=3 / 1) . \quad$ IR (neat): $2978(\mathrm{~m}), 1767(\mathrm{~s}), 1726(\mathrm{~s}), 1466(\mathrm{~m}), 1288(\mathrm{~m})$, 
$1204(\mathrm{~m}), 1142(\mathrm{~m}), 1070(\mathrm{~m}), 745(\mathrm{~m}) \mathrm{cm}^{-1} . \quad{ }^{1} \mathrm{H} \mathrm{NMR}\left(400 \mathrm{MHz}, \mathrm{CDCl}_{3}, \mathrm{rt}\right): \delta 7.88(\mathrm{~d}, J=7.6 \mathrm{~Hz}, 1 \mathrm{H})$, $7.67(\mathrm{dt}, J=7.6,1.2 \mathrm{~Hz}, 1 \mathrm{H}), 7.54-7.47(\mathrm{~m}, 2 \mathrm{H}), 5.52(\mathrm{dd}, J=10.0,1.6 \mathrm{~Hz}, 1 \mathrm{H}), 4.20(\mathrm{q}, J=7.2 \mathrm{~Hz}, 2 \mathrm{H})$, $2.36(\mathrm{dd}, J=14.8,1.6 \mathrm{~Hz}, 1 \mathrm{H}), 1.82(\mathrm{dd}, J=14.8,10.0 \mathrm{~Hz}, 1 \mathrm{H}), 1.40$ (s, 3H), 1.35 (s, 3H), 1.29 (t, $J=7.2$ $\mathrm{Hz}, 3 \mathrm{H}) ;{ }^{13} \mathrm{C}\left\{{ }^{1} \mathrm{H}\right\} \mathrm{NMR}\left(150 \mathrm{MHz}, \mathrm{CDCl}_{3}, \mathrm{rt}\right): \delta 177.4,170.5,150.6,134.2,129.3,125.8(2 \mathrm{C}), 122.0,79.3$, 61.0, 45.8, 41.8, 26.3, 25.4, 14.3. Calcd for $\mathrm{C}_{15} \mathrm{H}_{18} \mathrm{O}_{4}$ : C, 68.69; H, 6.92. Found: C, 68.68; H, 7.11.

\section{3-(2-Ethoxycarbonyl-2-phenylethyl)phthalide (3ab)}

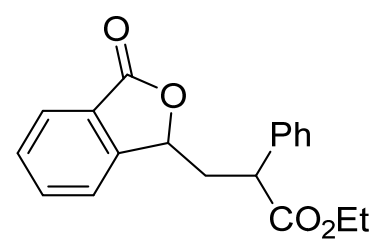

$3 a b$

A 1:1 mixture of diastereoisomers 3ab was obtained from $o$-vinylbenzoic acid (1a, $59.2 \mathrm{mg}, 0.4 \mathrm{mmol}$ ) and ethyl $\alpha$-bromophenylacetate $(\mathbf{2 b}, 194.5 \mathrm{mg}, 0.8 \mathrm{mmol})$ at $120{ }^{\circ} \mathrm{C}$ for $8 \mathrm{~h}$ as a colorless oil $(120.7 \mathrm{mg}$, $0.389 \mathrm{mmol}, 97 \%) . \quad R_{\mathrm{f}}=0.35$ (hexane/ethyl acetate = 3/1). IR (neat): $2980(\mathrm{~m}), 1767(\mathrm{~s}), 1732(\mathrm{~s}), 1466$ (m), 1287 (m), 1018 (m), 698 (m) cm ${ }^{-1} . \quad{ }^{1} \mathrm{H}$ NMR (600 MHz, $\left.\mathrm{CDCl}_{3}, \mathrm{rt}\right): \delta 7.88-7.86$ (m, 1H), 7.66-7.63 (m, 1H), 7.52-7.48 (m, 1.5H), 7.43-7.41 (m, 1H), 7.39-7.36 (m, 1.5H), 7.32-7.21 (m, 3H), 5.55 (dd, $J=$ $10.2,3.0 \mathrm{~Hz}, 0.5 \mathrm{H}), 5.13(\mathrm{dd}, J=10.2,3.0 \mathrm{~Hz}, 0.5 \mathrm{H}), 4.24-4.10(\mathrm{~m}, 1 \mathrm{H}), 4.10-3.99(\mathrm{~m}, 2 \mathrm{H}), 2.93(\mathrm{ddd}, J=$ $14.4,11.4,3.0 \mathrm{~Hz}, 0.5 \mathrm{H}), 2.57$ (ddd, $J=14.4,10.2,3.0 \mathrm{~Hz}, 0.5 \mathrm{H}$ ), 2.30 (ddd, $J=14.4,10.2,4.8 \mathrm{~Hz}, 0.5 \mathrm{H}$ ), $1.86(\mathrm{ddd}, J=14.4,10.2,3.6 \mathrm{~Hz}, 0.5 \mathrm{H}), 1.21(\mathrm{t}, J=7.2 \mathrm{~Hz}, 1.5 \mathrm{H}), 1.15(\mathrm{t}, J=7.2 \mathrm{~Hz}, 1.5 \mathrm{H}) ;{ }^{13} \mathrm{C}\left\{{ }^{1} \mathrm{H}\right\} \mathrm{NMR}$ (150 MHz, $\left.\mathrm{CDCl}_{3}, \mathrm{rt}\right): \delta 173.30,173.29,170.4,170.3,149.7,149.5,138.6,137.5,134.2,134.1,129.4,129.1$, 128.9 (2C), 128.4, 127.9, 127.6 (2C), 126.0, 125.9, 125.84, 125.79, 122.01, 121.99, 79.6, 78.5, 61.4, 61.2, 47.8, 47.5, 39.4, 38.7, 14.2, 14.1. Calcd for $\mathrm{C}_{19} \mathrm{H}_{18} \mathrm{O}_{4}$ : C, 73.53; H, 5.85. Found: C, 73.48; H, 5.82.

\section{3-[2-Ethoxycarbonyl-2-(p-methoxyphenyl)ethyl]phthalide (3ac)}

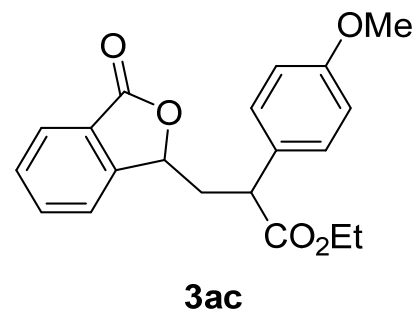

A 1:1 mixture of diastereoisomers 3ac was obtained from $o$-vinylbenzoic acid (1a, $59.2 \mathrm{mg}, 0.4 \mathrm{mmol}$ ) and ethyl $\alpha$-bromo-4-methoxyphenylacetate (2c, $218.5 \mathrm{mg}, 0.8 \mathrm{mmol}$ ) at $120^{\circ} \mathrm{C}$ for $8 \mathrm{~h}$ as a colorless oil $(113.2 \mathrm{mg}, 0.333 \mathrm{mmol}, 83 \%) . \quad R_{\mathrm{f}}=0.32$ (hexane/ethyl acetate = 3/1). IR (neat): $2980(\mathrm{~m}), 2361(\mathrm{~m})$, 
1771 (s), 1732 (s), 1514 (m), 1252 (m), 1179 (m), 1032 (m), 696 (m) cm ${ }^{-1} . \quad{ }^{1} \mathrm{H}$ NMR (600 MHz, $\left.\mathrm{CDCl}_{3}, \mathrm{rt}\right)$ : $\delta 7.89-7.87(\mathrm{~m}, 1 \mathrm{H}), 7.67-7.63(\mathrm{~m}, 1 \mathrm{H}), 7.53-7.48(\mathrm{~m}, 1.5 \mathrm{H}), 7.38(\mathrm{~d}, J=7.2 \mathrm{~Hz}, 0.5 \mathrm{H}), 7.35(\mathrm{~d}, J=8.4 \mathrm{~Hz}$, 1H), 7.19 (d, $J=8.4 \mathrm{~Hz}, 1 \mathrm{H}), 6.92(\mathrm{~d}, J=8.4 \mathrm{~Hz}, 1 \mathrm{H}), 6.82(\mathrm{~d}, J=8.4 \mathrm{~Hz}, 1 \mathrm{H}), 5.54$ (dd, $J=10.2,3.0 \mathrm{~Hz}$, $0.5 \mathrm{H}), 5.14(\mathrm{dd}, J=10.8,3.0 \mathrm{~Hz}, 0.5 \mathrm{H}), 4.24-3.99(\mathrm{~m}, 2 \mathrm{H}), 3.99-3.95(\mathrm{~m}, 1 \mathrm{H}), 3.81(\mathrm{~s}, 1.5 \mathrm{H}), 3.77(\mathrm{~s}, 1.5 \mathrm{H})$, $2.90(\mathrm{ddd}, J=14.4,11.4,3.0 \mathrm{~Hz}, 0.5 \mathrm{H}$ ), 2.53 (ddd, $J=14.4,10.8,3.0 \mathrm{~Hz}, 0.5 \mathrm{H}), 2.26$ (ddd, $J=14.4,10.8$, $4.8 \mathrm{~Hz}, 0.5 \mathrm{H}), 1.84$ (ddd, $J=14.4,10.2,4.2 \mathrm{~Hz}, 0.5 \mathrm{H}), 1.22(\mathrm{t}, J=7.2 \mathrm{~Hz}, 1.5 \mathrm{H}), 1.16$ (t, $J=7.2 \mathrm{~Hz}, 1.5 \mathrm{H}$ ); ${ }^{13} \mathrm{C}\left\{{ }^{1} \mathrm{H}\right\}$ NMR (150 MHz, CDCl $\left.3, \mathrm{rt}\right): \delta 173.6$ (2C), 170.5, 170.4, 159.3, 159.0, 149.8, 149.7, 134.3, 134.1, 130.7, 129.51, 129.45, 129.4, 128.7 (2C), 126.0, 125.92, 125.90, 125.8, 122.03, 122.01, 114.5, 114.3, 79.7, 78.6, 61.4, 61.2, 55.42, 55.39, 47.0, 46.7, 39.5, 38.9, 14.22, 14.18. Calcd for $\mathrm{C}_{20} \mathrm{H}_{20} \mathrm{O}_{5}: \mathrm{C}, 70.58 ; \mathrm{H}, 5.92$. Found: C, 70.51; H, 5.82 .

\section{3-[2-Ethoxycarbonyl-2-[p-(trifluoromethyl)phenyl]ethyl]phthalide (3ad)}

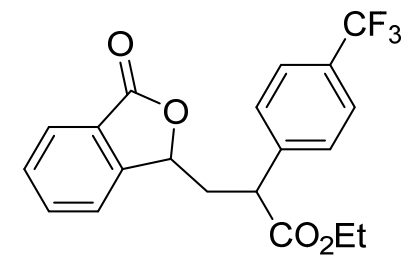

3ad

A 1:1 mixture of diastereoisomers 3ad was obtained from $o$-vinylbenzoic acid (1a, $59.2 \mathrm{mg}, 0.4 \mathrm{mmol}$ ) and ethyl $\alpha$-bromo-4-trifluoromethylphenylacetate (2d, $248.8 \mathrm{mg}, 0.8 \mathrm{mmol}$ ) at $120^{\circ} \mathrm{C}$ for $8 \mathrm{~h}$ as a colorless oil (122.9 mg, $0.325 \mathrm{mmol}, 81 \%) . \quad R_{\mathrm{f}}=0.22$ (hexane/ethyl acetate = 5/1). IR (neat): $2984(\mathrm{~m}), 2359(\mathrm{~s})$, 1771 (s), 1732 (s), 1618 (m), 1327 (s), 1167 (m), 1018 (m), 764 (m) cm $\left.{ }^{-1} . \quad{ }^{1} \mathrm{H} \mathrm{NMR} 600 \mathrm{MHz}, \mathrm{CDCl}_{3}, \mathrm{rt}\right)$ : $\delta 7.87-7.85(\mathrm{~m}, 1 \mathrm{H}), 7.66-7.62(\mathrm{~m}, 2 \mathrm{H}), 7.56-7.49(\mathrm{~m}, 3 \mathrm{H}), 7.45(\mathrm{~d}, J=7.8 \mathrm{~Hz}, 0.5 \mathrm{H}), 7.39(\mathrm{~d}, J=7.8 \mathrm{~Hz}$, $0.5 \mathrm{H}), 7.37(\mathrm{~d}, J=8.4 \mathrm{~Hz}, 1 \mathrm{H}), 5.56(\mathrm{dd}, J=9.6,3.0 \mathrm{~Hz}, 0.5 \mathrm{H}), 5.10(\mathrm{dd}, J=10.2,3.0 \mathrm{~Hz}, 0.5 \mathrm{H}), 4.24-4.11$ (m, 1H), 4.09-3.99 (m, 2H), $2.96(\mathrm{ddd}, J=14.4,11.4,3.0 \mathrm{~Hz}, 0.5 \mathrm{H}), 2.57$ (ddd, $J=15.0,10.2,3.0 \mathrm{~Hz}, 0.5 \mathrm{H})$, $2.35(\mathrm{ddd}, J=15.0,10.2,5.4 \mathrm{~Hz}, 0.5 \mathrm{H}), 1.87$ (ddd, $J=14.4,9.6,3.6 \mathrm{~Hz}, 0.5 \mathrm{H}), 1.21(\mathrm{t}, J=7.2 \mathrm{~Hz}, 1.5 \mathrm{H})$, $1.14(\mathrm{t}, J=7.2 \mathrm{~Hz}, 1.5 \mathrm{H}) ;{ }^{13} \mathrm{C}\left\{{ }^{1} \mathrm{H}\right\} \mathrm{NMR}\left(150 \mathrm{MHz}, \mathrm{CDCl}_{3}, \mathrm{rt}\right): \delta 172.57,172.56,170.2,170.1,149.3,149.1$, 142.6, 141.5, 134.3, 134.2, $130.2\left(\mathrm{q},{ }^{2} J_{\mathrm{C}-\mathrm{F}}=33 \mathrm{~Hz}\right), 129.8\left(\mathrm{q},{ }^{2} J_{\mathrm{C}-\mathrm{F}}=33 \mathrm{~Hz}\right), 129.52,129.50,128.9,128.1$, $126.1\left(\mathrm{q},{ }^{3} J_{\mathrm{C}-\mathrm{F}}=4.5 \mathrm{~Hz}\right), 125.92,125.87(2 \mathrm{C}), 125.85\left(\mathrm{q},{ }^{3} J_{\mathrm{C}-\mathrm{F}}=4.5 \mathrm{~Hz}\right), 125.7,124.05\left(\mathrm{q},{ }^{1} J_{\mathrm{C}-\mathrm{F}}=270 \mathrm{~Hz}\right)$, $124.01\left(\mathrm{q},{ }^{1} J_{\mathrm{C}-\mathrm{F}}=270 \mathrm{~Hz}\right), 122.01,121.95,79.2,78.2,61.7,61.5,47.6,47.3,39.0,38.4,14.1,14.0 ;{ }^{19} \mathrm{~F}\left\{{ }^{1} \mathrm{H}\right\}$ NMR (564 MHz, CDCl3, rt): $\delta-62.6,-62.7$. Calcd for $\mathrm{C}_{20} \mathrm{H}_{17} \mathrm{~F}_{3} \mathrm{O}_{4}: \mathrm{C}, 63.49 ; \mathrm{H}, 4.53$. Found: $\mathrm{C}, 63.50 ; \mathrm{H}$, 4.44 . 


\section{3-[2-Ethoxycarbonyl-2-(p-fluorophenyl)ethyl]phthalide (3ae)}

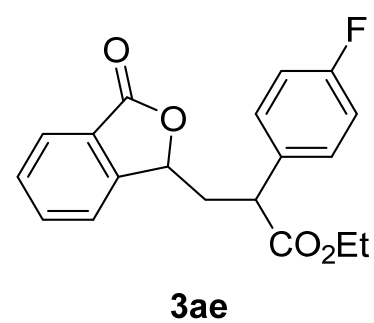

A 1:1 mixture of diastereoisomers 3ae was obtained from $o$-vinylbenzoic acid (1a, $59.2 \mathrm{mg}, 0.4 \mathrm{mmol}$ ) and ethyl $\alpha$-bromo-4-fluorophenylacetate (2e, $208.9 \mathrm{mg}, 0.8 \mathrm{mmol})$ at $120^{\circ} \mathrm{C}$ for $8 \mathrm{~h}$ as a colorless oil $(103.8$ $\mathrm{mg}, 0.316 \mathrm{mmol}, 79 \%) . \quad R_{\mathrm{f}}=0.26$ (hexane/ethyl acetate $\left.=4 / 1\right) . \quad$ IR (neat): $2982(\mathrm{~m}), 1771(\mathrm{~s}), 1732(\mathrm{~s})$, 1510 (m), 1225 (m), $1016(\mathrm{~m}), 696(\mathrm{~m}) \mathrm{cm}^{-1}$. ${ }^{1} \mathrm{H}$ NMR (600 MHz, $\left.\mathrm{CDCl}_{3}, \mathrm{rt}\right): \delta 7.87$ (d, $\left.J=7.8 \mathrm{~Hz}, 1 \mathrm{H}\right)$, $7.65(\mathrm{t}, J=7.8 \mathrm{~Hz}, 1 \mathrm{H}), 7.51$ (t, $J=7.8 \mathrm{~Hz}, 1 \mathrm{H}), 7.47$ (d, $J=7.8 \mathrm{~Hz}, 0.5 \mathrm{H}), 7.40-7.38$ (m, 1.5H), 7.23-7.21 (m, 1H), $7.06(\mathrm{t}, J=8.4 \mathrm{~Hz}, 1 \mathrm{H}), 6.96(\mathrm{t}, J=8.4 \mathrm{~Hz}, 1 \mathrm{H}), 5.54(\mathrm{dd}, J=10.2,3.0 \mathrm{~Hz}, 0.5 \mathrm{H}), 5.12(\mathrm{dd}, J=$ 10.2, 3.0 Hz, 0.5H), 4.22-4.10 (m, 1H), 4.09-3.97 (m, 2H), 2.91 (ddd, $J=14.4,11.4,3.0 \mathrm{~Hz}, 0.5 \mathrm{H}), 2.53$ (ddd, $J=15.0,10.2,3.0 \mathrm{~Hz}, 0.5 \mathrm{H}), 2.29$ (ddd, $J=15.0,10.2,4.8 \mathrm{~Hz}, 0.5 \mathrm{H}), 1.84$ (ddd, $J=14.4,10.2,3.6$ $\mathrm{Hz}, 0.5 \mathrm{H}), 1.21(\mathrm{t}, J=7.2 \mathrm{~Hz}, 1.5 \mathrm{H}), 1.14(\mathrm{t}, J=7.2 \mathrm{~Hz}, 1.5 \mathrm{H}) ;{ }^{13} \mathrm{C}\left\{{ }^{1} \mathrm{H}\right\} \mathrm{NMR}\left(150 \mathrm{MHz}, \mathrm{CDCl}_{3}, \mathrm{rt}\right): \delta$ 173.18, 173.15, 170.3, 170.2, $162.4\left(\mathrm{~d},{ }^{1} J_{\mathrm{C}-\mathrm{F}}=245 \mathrm{~Hz}\right), 162.2\left(\mathrm{~d},{ }^{1} J_{\mathrm{C}-\mathrm{F}}=245 \mathrm{~Hz}\right), 149.5,149.3,134.4(\mathrm{~d}$, $\left.{ }^{4} J_{\mathrm{C}-\mathrm{F}}=2.9 \mathrm{~Hz}\right), 134.3,134.2,133.2\left(\mathrm{~d},{ }^{4} J_{\mathrm{C}-\mathrm{F}}=3.5 \mathrm{~Hz}\right), 130.0\left(\mathrm{~d},{ }^{3} J_{\mathrm{C}-\mathrm{F}}=8.1 \mathrm{~Hz}\right), 129.45,129.43,129.2(\mathrm{~d}$, $\left.{ }^{3} J_{\mathrm{C}-\mathrm{F}}=8.1 \mathrm{~Hz}\right), 125.92,125.90,125.87,125.8,121.99,121.97,116.0\left(\mathrm{~d},{ }^{2} J_{\mathrm{C}-\mathrm{F}}=37 \mathrm{~Hz}\right), 115.8\left(\mathrm{~d},{ }^{2} J_{\mathrm{C}-\mathrm{F}}=37\right.$ $\mathrm{Hz}), 79.4,78.4,61.5,61.3,47.1,46.8,39.3,38.7,14.13,14.09 ;{ }^{19} \mathrm{~F}\left\{{ }^{1} \mathrm{H}\right\}$ NMR (564 MHz, CDCl3, rt): $\delta-$ 98.5, -98.1. Calcd for $\mathrm{C}_{19} \mathrm{H}_{17} \mathrm{FO}_{4}$ : C, 69.50; H, 5.22. Found: C, 69.49; H, 5.05.

\section{3-[2-(p-Chlorophenyl)-2-(ethoxycarbonyl)ethyl]phthalide (3af)}

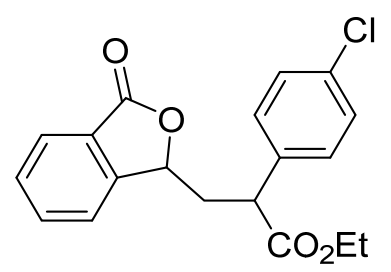

3af

A 1:1 mixture of diastereoisomers 3af was obtained from $o$-vinylbenzoic acid (1a, $59.2 \mathrm{mg}, 0.4 \mathrm{mmol})$ and ethyl $\alpha$-bromo-4-chlorophenylacetate (2f, $222.0 \mathrm{mg}, 0.8 \mathrm{mmol})$ at $120^{\circ} \mathrm{C}$ for $8 \mathrm{~h}$ as a colorless oil $(123.9 \mathrm{mg}$, $0.359 \mathrm{mmol}, 90 \%) . \quad R_{\mathrm{f}}=0.23$ (hexane/ethyl acetate $\left.=5 / 1\right) . \quad$ IR (neat): $2982(\mathrm{~m}), 1769(\mathrm{~s}), 1730(\mathrm{~s}), 1466$ (m), $1287(\mathrm{~m}), 1173(\mathrm{~m}), 762(\mathrm{~m}) \mathrm{cm}^{-1} . \quad{ }^{1} \mathrm{H} \mathrm{NMR}\left(600 \mathrm{MHz}, \mathrm{CDCl}_{3}, \mathrm{rt}\right): \delta 7.89$ (d, $\left.J=7.8 \mathrm{~Hz}, 1 \mathrm{H}\right), 7.66(\mathrm{t}$, $J=7.8 \mathrm{~Hz}, 1 \mathrm{H}), 7.53(\mathrm{t}, J=7.8 \mathrm{~Hz}, 1 \mathrm{H}), 7.48(\mathrm{~d}, J=7.8 \mathrm{~Hz}, 0.5 \mathrm{H}), 7.39-7.35(\mathrm{~m}, 2.5 \mathrm{H}), 7.26(\mathrm{~d}, J=8.4 \mathrm{~Hz}$, 
$1 \mathrm{H}), 7.20(\mathrm{~d}, J=8.4 \mathrm{~Hz}, 1 \mathrm{H}), 5.55(\mathrm{dd}, J=9.6,3.0 \mathrm{~Hz}, 0.5 \mathrm{H}), 5.12(\mathrm{dd}, J=10.2,3.0 \mathrm{~Hz}, 0.5 \mathrm{H}), 4.24-4.11$ (m, 1H), 4.10-3.98 (m, 2H), 2.92 (ddd, $J=14.4,11.4,3.0 \mathrm{~Hz}, 0.5 \mathrm{H}), 2.53$ (ddd, $J=14.4,10.2,3.0 \mathrm{~Hz}, 0.5 \mathrm{H}$ ), 2.30 (ddd, $J=14.4,10.2,4.8 \mathrm{~Hz}, 0.5 \mathrm{H}), 1.84$ (ddd, $J=14.4,9.6,3.6 \mathrm{~Hz}, 0.5 \mathrm{H}), 1.22(t, J=7.2 \mathrm{~Hz}, 1.5 \mathrm{H})$, $1.16(\mathrm{t}, J=7.2 \mathrm{~Hz}, 1.5 \mathrm{H}) ;{ }^{13} \mathrm{C}\left\{{ }^{1} \mathrm{H}\right\} \mathrm{NMR}\left(150 \mathrm{MHz}, \mathrm{CDCl}_{3}, \mathrm{rt}\right): \delta 173.02,172.99,170.4,170.3,149.5,149.3$, 137.1, 136.0, 134.3, 134.2, 133.9, 133.6, 129.8, 129.53, 129.51, 129.4, 129.14, 129.07 (2C), 126.01, 125.97, $125.8,122.0$ (2C), 78.4, 78.3, 61.6, 61.5, 47.3, 47.0, 39.3, 38.6, 14.2, 14.1. Calcd for $\mathrm{C}_{19} \mathrm{H}_{17} \mathrm{ClO}_{4}$ : C, 66.19; H, 4.97. Found: C, 66.55; H, 4.83 .

\section{3-[1-(Ethoxycarbonyl)cyclobutylmethyl]phthalide (3ag)}

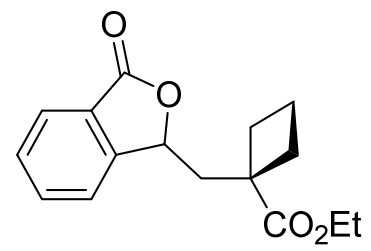

3ag

The product 3ag was obtained from $o$-vinylbenzoic acid $(1 \mathrm{a}, 59.2 \mathrm{mg}, 0.4 \mathrm{mmol}$ ) and ethyl 1bromocyclobuthylcalboxlate $(2 \mathrm{~g}, 218.5 \mathrm{mg}, 0.8 \mathrm{mmol})$ at $120^{\circ} \mathrm{C}$ for $8 \mathrm{~h}$ as a colorless oil $(78.0 \mathrm{mg}, 0.284$ mmol, 71\%). $\quad R_{\mathrm{f}}=0.36$ (hexane/ethyl acetate =3/1). IR (neat): $2982(\mathrm{~m}), 1767(\mathrm{~s}), 1724(\mathrm{~s}), 1206(\mathrm{~m})$, $696(\mathrm{~m}) \mathrm{cm}^{-1} . \quad{ }^{1} \mathrm{H}$ NMR $\left(600 \mathrm{MHz}, \mathrm{CDCl}_{3}, \mathrm{rt}\right): \delta 7.89(\mathrm{~d}, J=7.8 \mathrm{~Hz}, 1 \mathrm{H}), 7.68(\mathrm{td}, J=7.8,1.2 \mathrm{~Hz}, 1 \mathrm{H})$, 7.53 (t, $J=7.8 \mathrm{~Hz}, 1 \mathrm{H}), 7.47$ (dd, $J=7.8,1.2 \mathrm{~Hz}, 1 \mathrm{H}), 5.48$ (dd, $J=9.6,2.4 \mathrm{~Hz}, 1 \mathrm{H}), 4.22$ (q, $J=7.2 \mathrm{~Hz}$, 2H), 2.58-2.52 (m, 3H), 2.24-2.20 (m, 1H), $2.14(\mathrm{dd}, J=14.4,9.6 \mathrm{~Hz}, 1 \mathrm{H}), 2.05-1.94(\mathrm{~m}, 3 \mathrm{H}), 1.31$ (t, $J=$ $7.2 \mathrm{~Hz}, 3 \mathrm{H}) ;{ }^{13} \mathrm{C}\left\{{ }^{1} \mathrm{H}\right\} \mathrm{NMR}\left(100 \mathrm{MHz}, \mathrm{CDCl}_{3}, \mathrm{rt}\right): \delta 176.5,170.4,150.2,134.1,129.3,126.0,125.9,122.0$, 79.6, 61.0, 46.4, 43.3, 31.0, 30.4, 16.1, 14.4. Calcd for $\mathrm{C}_{16} \mathrm{H}_{18} \mathrm{O}_{4}$ : C, 70.06; H, 6.61. Found: C, 69.95; H, 6.60 .

\section{3-(2-Cyano-2-phenylethyl)phthalide (3ah)}

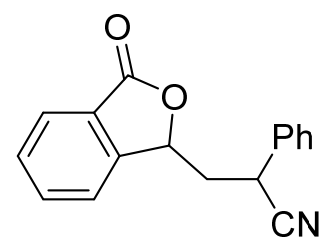

3ah

Major diastereomer 3ah was obtained from $o$-vinylbenzoic acid (1a, $59.2 \mathrm{mg}, 0.4 \mathrm{mmol}$ ) and 
bromo(phenyl)acetonitrile ( $2 \mathrm{~h}, 222.0 \mathrm{mg}, 0.8 \mathrm{mmol})$ at $120^{\circ} \mathrm{C}$ for $8 \mathrm{~h}$ as dark yellow solid $(34.5 \mathrm{mg}, 0.131$ mmol, 33\%). $\quad R_{\mathrm{f}}=0.16$ (hexane/ethyl acetate $\left.=3 / 1\right) . \quad$ M.p. $115-116{ }^{\circ} \mathrm{C} . \quad \operatorname{IR}(\mathrm{KBr}): 3030(\mathrm{w}), 2359(\mathrm{~m})$, 2245 (w), 1749 (s), 1468 (m), 1283 (m), 1016 (m), 702 (m) cm ${ }^{-1} . \quad{ }^{1} \mathrm{H}$ NMR (600 MHz, $\mathrm{CDCl}_{3}, \mathrm{rt}$ ): $\delta 7.92$ (d, $J=7.2 \mathrm{~Hz}, 1 \mathrm{H}), 7.68$ (td, $J=7.2,1.2 \mathrm{~Hz}, 1 \mathrm{H}), 7.56(\mathrm{t}, J=7.2 \mathrm{~Hz}, 1 \mathrm{H}), 7.51-7.45(\mathrm{~m}, 4 \mathrm{H}), 7.41$ (tt, $J=$ 7.2, $1.2 \mathrm{~Hz}, 1 \mathrm{H}), 7.36(\mathrm{dd}, J=7.2,1.2 \mathrm{~Hz}, 1 \mathrm{H}), 5.14(\mathrm{dd}, J=10.8,3.0 \mathrm{~Hz}, 1 \mathrm{H}), 4.20$ (dd, $J=10.8,4.8 \mathrm{~Hz}$, 1H), 2.54 (ddd, $J=14.4,10.8,3.0 \mathrm{~Hz}, 1 \mathrm{H}), 2.37$ (ddd, $J=14.4,10.8,4.8 \mathrm{~Hz}, 1 \mathrm{H}), ;{ }^{13} \mathrm{C}\left\{{ }^{1} \mathrm{H}\right\} \mathrm{NMR}(150 \mathrm{MHz}$, $\left.\mathrm{CDCl}_{3}, \mathrm{rt}\right): \delta 169.8,148.4,134.6,133.9,130.0,129.7,129.0,128.2,126.3,125.8,121.8,120.4,76.8,40.8$, 33.7. HRMS (ESI) $\mathrm{m} / \mathrm{z}$ : $[\mathrm{M}+\mathrm{Na}]^{+}$Calcd for $\mathrm{C}_{17} \mathrm{H}_{13} \mathrm{NNaO}_{2}$ 286.0844; Found: 286.0850 .

Minor diastereomer 3ah was obtained as dark yellow solid $(26.6 \mathrm{mg}, 0.101 \mathrm{mmol}, 25 \%) . \quad R_{\mathrm{f}}=0.22$ (hexane/ethyl acetate =3/1). M.p. $115-116^{\circ} \mathrm{C} . \quad$ IR (KBr): 3032 (w), 2361 (w), 2241 (w), 1769 (s), 1466 (w), 1287 (m), 1024 (m), 700 (m) $\mathrm{cm}^{-1}$. ${ }^{1} \mathrm{H}$ NMR (600 MHz, $\left.\mathrm{CDCl}_{3}, \mathrm{rt}\right): \delta 7.93$ (d, J=7.8 Hz, 1H), 7.69 (dt, $J=7.8,1.2 \mathrm{~Hz}, 1 \mathrm{H}), 7.57$ (t, $J=7.8 \mathrm{~Hz}, 1 \mathrm{H}), 7.46(\mathrm{dd}, J=7.8,0.6 \mathrm{~Hz}, 1 \mathrm{H}), 7.40-7.32$ (m, 5H), 5.80 (dd, $J=10.8,2.4 \mathrm{~Hz}, 1 \mathrm{H}), 4.33(\mathrm{dd}, J=12.0,3.6 \mathrm{~Hz}, 1 \mathrm{H}), 2.62$ (ddd, $J=14.4,12.0,2.4 \mathrm{~Hz}, 1 \mathrm{H}), 2.00$ (ddd, $J=$ 14.4, 10.8, 3.6 Hz, 1H); ${ }^{13} \mathrm{C}\left\{{ }^{1} \mathrm{H}\right\}$ NMR (150 MHz, $\left.\mathrm{CDCl}_{3}, \mathrm{rt}\right): \delta 169.9,148.5,134.9,134.6,129.9,129.5$, 128.7, 127.2, 126.3, 125.7, 121.8, 119.9, 78.3, 42.3, 35.0. HRMS (ESI) $\mathrm{m} / \mathrm{z}:[\mathrm{M}+\mathrm{Na}]^{+}$Calcd for $\mathrm{C}_{17} \mathrm{H}_{13} \mathrm{NNaO}_{2} 286.0844$; Found: 286.0841 .

\section{3-(2-Cinnamyloxycarbonyl-2-methylpropyl)phthalide (3ai)}

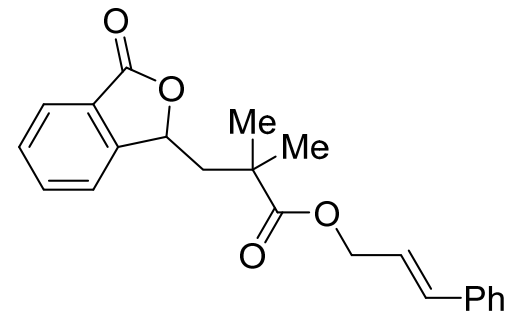

3ai

The product 3ai was obtained from $o$-vinylbenzoic acid (1a, $59.2 \mathrm{mg}, 0.4 \mathrm{mmol})$ and cinnamyl $\alpha$-bromoisobuthyrate $(2 \mathbf{i}, 226.5 \mathrm{mg}, 0.8 \mathrm{mmol})$ at $80^{\circ} \mathrm{C}$ for $16 \mathrm{~h}$ as a colorless oil $(67.5 \mathrm{mg}, 0.193 \mathrm{mmol}, 48 \%)$. $\quad R_{\mathrm{f}}$ $=0.32($ hexane/ethyl acetate = 3/1). IR (neat): $2976(\mathrm{~m}), 1767(\mathrm{~s}), 1728(\mathrm{~s}), 1466(\mathrm{~m}), 1288(\mathrm{~m}), 1138(\mathrm{~m})$, $745(\mathrm{~m}) \mathrm{cm}^{-1} . \quad{ }^{1} \mathrm{H} \mathrm{NMR}\left(600 \mathrm{MHz}, \mathrm{CDCl}_{3}, \mathrm{rt}\right): \delta 7.87(\mathrm{~d}, J=7.8 \mathrm{~Hz}, 1 \mathrm{H}), 7.64(\mathrm{t}, J=7.8 \mathrm{~Hz}, 1 \mathrm{H}), 7.50$ (t, $J=7.8 \mathrm{~Hz}, 1 \mathrm{H}), 7.47$ (d, $J=7.8 \mathrm{~Hz}, 1 \mathrm{H}), 7.40$ (d, $J=7.8 \mathrm{~Hz}, 2 \mathrm{H}), 7.32$ (t, $J=7.8 \mathrm{~Hz}, 2 \mathrm{H}), 7.25$ (t, $J=7.8$ $\mathrm{Hz}, 1 \mathrm{H}), 6.67(\mathrm{~d}, J=15.6 \mathrm{~Hz}, 1 \mathrm{H}), 6.32(\mathrm{dt}, J=15.6,6.6 \mathrm{~Hz}, 1 \mathrm{H}), 5.54(\mathrm{~d}, J=10.2 \mathrm{~Hz}, 1 \mathrm{H}), 4.84-4.77(\mathrm{~m}$, 2H), $2.37(\mathrm{dd}, J=15.0,1.8 \mathrm{~Hz}, 1 \mathrm{H}), 1.87(\mathrm{dd}, J=15.0,10.2 \mathrm{~Hz}, 1 \mathrm{H}), 1.44(\mathrm{~s}, 3 \mathrm{H}), 1.39(\mathrm{~s}, 3 \mathrm{H}) ;{ }^{13} \mathrm{C}\left\{{ }^{1} \mathrm{H}\right\}$ NMR (150 MHz, $\left.\mathrm{CDCl}_{3}, \mathrm{rt}\right): \delta 177.1,170.4,150.5,136.3,134.4,134.2,129.2,128.7,128.2,126.7,125.8$, 125.7, 123.3, 122.0, 79.1, 65.6, 45.8, 41.9, 26.1, 25.5. HRMS (ESI) $\mathrm{m} / \mathrm{z}$ : $[\mathrm{M}+\mathrm{Na}]^{+}$Calcd for $\mathrm{C}_{22} \mathrm{H}_{22} \mathrm{NaO}_{4}$ 
373.1416; Found: 373.1391.

\section{3-(2,2-Diethoxycarbonylethyl)phthalide (3aj)}

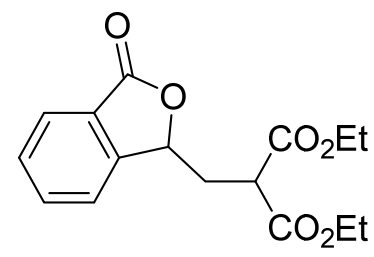

3aj

The product 3aj was obtained from $o$-vinylbenzoic acid (1a, $59.2 \mathrm{mg}, 0.4 \mathrm{mmol}$ ) and diethyl bromomalonate ( $2 \mathbf{j}, 226.5 \mathrm{mg}, 0.8 \mathrm{mmol})$ at $140{ }^{\circ} \mathrm{C}$ for $8 \mathrm{~h}$ as a colorless oil $(81.0 \mathrm{mg}, 0.264 \mathrm{mmol}, 66 \%)$. $R_{\mathrm{f}}=0.20$ (hexane/ethyl acetate =3/1). IR (neat): $2984(\mathrm{~m}), 1771(\mathrm{~s}), 1749(\mathrm{~s}), 1732(\mathrm{~s}), 1287(\mathrm{~s}), 694(\mathrm{~m})$ $\mathrm{cm}^{-1} . \quad{ }^{1} \mathrm{H}$ NMR (600 MHz, $\left.\mathrm{CDCl}_{3}, \mathrm{rt}\right): \delta 7.91$ (d, $\left.J=7.8 \mathrm{~Hz}, 1 \mathrm{H}\right), 7.70(\mathrm{dt}, J=7.8 \mathrm{~Hz}, 1.2 \mathrm{~Hz}, 1 \mathrm{H}), 7.55$ (t, $J=7.8 \mathrm{~Hz} 1 \mathrm{H}), 7.50(\mathrm{dd}, J=7.8 \mathrm{~Hz}, 1.2 \mathrm{~Hz}, 1 \mathrm{H}), 5.55(\mathrm{dd}, J=9.0 \mathrm{~Hz}, 3.0 \mathrm{~Hz}, 1 \mathrm{H}), 4.17-4.09(\mathrm{~m}, 2 \mathrm{H})$, $4.30-4.23(\mathrm{~m}, 2 \mathrm{H}), 3.70(\mathrm{dd}, J=9.6 \mathrm{~Hz}, 4.8 \mathrm{~Hz}, 1 \mathrm{H}), 2.80-2.75$ (m, 1H), 2.19-2.14 (m, 1H), 1.31 (t, $J=7.2$ $\mathrm{Hz}, 3 \mathrm{H}), 1.23(\mathrm{t}, J=7.2 \mathrm{~Hz}, 3 \mathrm{H}) ;{ }^{13} \mathrm{C}\left\{{ }^{1} \mathrm{H}\right\} \mathrm{NMR}\left(150 \mathrm{MHz}, \mathrm{CDCl}_{3}, \mathrm{rt}\right): \delta 170.1,168.91,168.88,149.0,134.4$, 129.7, 126.1, 126.0, 122.2, 78.8, 62.1, 62.0, 48.1, 34.1, 14.2, 14.1. Calcd for $\mathrm{C}_{16} \mathrm{H}_{18} \mathrm{O}_{6}: \mathrm{C}, 62.74 ; \mathrm{H}, 5.92$. Found: C, 62.42; H, 5.73.

\section{3-(2,2-Diethoxycarbonylpropyl)phthalide (3ak)}

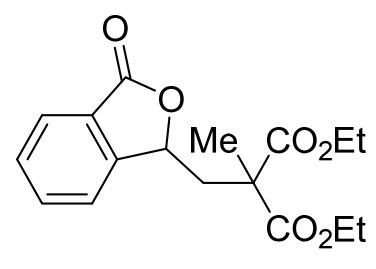

$3 a k$

The product 3ak was obtained from $o$-vinylbenzoic acid (1a, $59.2 \mathrm{mg}, 0.4 \mathrm{mmol}$ ) and diethyl 2-bromo-2methylmalonate (2k, $226.5 \mathrm{mg}, 0.8 \mathrm{mmol})$ at $140{ }^{\circ} \mathrm{C}$ for $8 \mathrm{~h}$ as white solid $(104.9 \mathrm{mg}, 0.327 \mathrm{mmol}, 82 \%)$. $R_{\mathrm{f}}=0.34$ (hexane/ethyl acetate $=3 / 1$ ). M.p. $96-97{ }^{\circ} \mathrm{C} . \quad \mathrm{IR}(\mathrm{KBr}): 2990(\mathrm{~m}), 1761$ (s), $1748(\mathrm{~m}), 1728$ (s), 1279 (m), $754(\mathrm{w}) \mathrm{cm}^{-1} . \quad{ }^{1} \mathrm{H}$ NMR (600 MHz, $\left.\mathrm{CDCl}_{3}, \mathrm{rt}\right): \delta 7.89$ (d, $\left.J=7.8 \mathrm{~Hz}, 1 \mathrm{H}\right), 7.69$ (dt, $J=7.8,1.2$ $\mathrm{Hz}, 1 \mathrm{H}), 7.55-7.52(\mathrm{~m}, 2 \mathrm{H}), 5.61(\mathrm{~d}, J=10.2 \mathrm{~Hz}, 1 \mathrm{H}), 4.31-4.22(\mathrm{~m}, 4 \mathrm{H}), 2.73(\mathrm{dd}, J=15.0,1.2 \mathrm{~Hz}, 1 \mathrm{H})$, $2.09(\mathrm{dd}, J=15.0 .10 .2 \mathrm{~Hz}, 1 \mathrm{H}), 1.65(\mathrm{~s}, 3 \mathrm{H}), 1.30(\mathrm{t}, J=7.2 \mathrm{~Hz}, 3 \mathrm{H}), 1.27(\mathrm{t}, J=7.2 \mathrm{~Hz}, 3 \mathrm{H}) ;{ }^{13} \mathrm{C}\left\{{ }^{1} \mathrm{H}\right\}$ NMR (150 MHz, $\left.\mathrm{CDCl}_{3}, \mathrm{rt}\right): \delta 171.83,171.77,170.1,150.1,134.3,129.5,125.9,125.8,122.2,78.3,62.0$, 61.9, 52.7, 41.9, 20.5, 14.2, 14.1. Calcd for $\mathrm{C}_{17} \mathrm{H}_{20} \mathrm{O}_{6}$ : C, 63.74; H, 6.29. Found: C, 63.47; H, 6.47. 


\section{3-(2-Ethoxycarbonyl-2-methylpropyl)-3-phenylphthalide (3ba)}

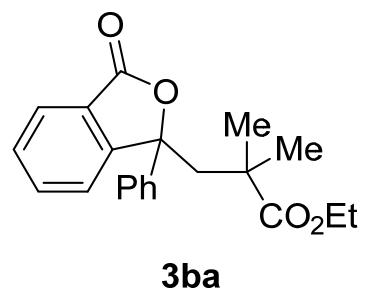

The product 3ba was obtained from 2-(1-phenylethenyl)benzoic acid (1b, $89.7 \mathrm{mg}, 0.4 \mathrm{mmol})$ and ethyl $\alpha$-bromoisobutylate (2a, $156.0 \mathrm{mg}, 0.8 \mathrm{mmol}$ ) at $80^{\circ} \mathrm{C}$ for $16 \mathrm{~h}$ as white solid $(53.8 \mathrm{mg}, 0.159 \mathrm{mmol}, 40 \%$ ). $R_{\mathrm{f}}=0.22($ hexane/ethyl acetate $=5 / 1) . \quad$ M.p. $118-119^{\circ} \mathrm{C} . \quad$ IR $(\mathrm{KBr}): 2982(\mathrm{~m}), 1763(\mathrm{~s}), 1721(\mathrm{~s}), 1252$ (m), $708(\mathrm{~s}) \mathrm{cm}^{-1} . \quad{ }^{1} \mathrm{H}$ NMR (400 MHz, $\left.\mathrm{CDCl}_{3}, \mathrm{rt}\right): \delta 7.86-7.84(\mathrm{~m}, 1 \mathrm{H}), 7.65-7.54(\mathrm{~m}, 4 \mathrm{H}), 7.50-7.46(\mathrm{~m}$, 1H), 7.36-7.31 (m, 2H), 7.29-7.24 (m, 1H), 4.01-3.89 (m, 2H), 2.91 (d, $J=15.2 \mathrm{~Hz}, 1 \mathrm{H}), 2.59$ (d, $J=15.2$ $\mathrm{Hz}, 1 \mathrm{H}), 1.18(\mathrm{t}, J=7.2 \mathrm{~Hz}, 3 \mathrm{H}), 1.09(\mathrm{~s}, 3 \mathrm{H}), 1.02(\mathrm{~s}, 3 \mathrm{H}) ;{ }^{13} \mathrm{C}\left\{{ }^{1} \mathrm{H}\right\} \mathrm{NMR}\left(100 \mathrm{MHz}, \mathrm{CDCl}_{3}, \mathrm{rt}\right): \delta 177.0$, 169.7, 153.7, 141.6, 134.2, 129.3, 128.8, 128.1, 126.1, 125.0, 124.8, 122.7, 88.8, 60.9, 49.7, 41.8, 27.0, 26.8, 14.1. Calcd for $\mathrm{C}_{21} \mathrm{H}_{22} \mathrm{O}_{4}$ : C, 74.54; $\mathrm{H}, 6.55$. Found: $\mathrm{C}, 74.39 ; \mathrm{H}, 6.69$.

\section{3-(2-Ethoxycarbonyl-2-methylpropyl)-6-fluorophthalide (3ca)}

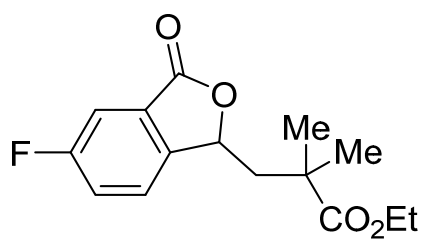

$3 \mathrm{ca}$

The product 3ca was obtained from 5-fruoro-o-vinylbenzoic acid (1c, $66.5 \mathrm{mg}, 0.4 \mathrm{mmol}$ ) and ethyl $\alpha$ bromoisobutylate (2a, $156.0 \mathrm{mg}, 0.8 \mathrm{mmol})$ at $80{ }^{\circ} \mathrm{C}$ for $16 \mathrm{~h}$ as a colorless oil $(80.2 \mathrm{mg}, 0.286 \mathrm{mmol}, 72 \%)$. $R_{\mathrm{f}}=0.38$ (hexane/ethyl acetate $\left.=3 / 1\right) . \quad$ IR (neat): $2980(\mathrm{~m}), 1771(\mathrm{~s}), 1728(\mathrm{~s}), 1491(\mathrm{~s}), 1267(\mathrm{~m}), 1142$ (m), $777(\mathrm{~m}) \mathrm{cm}^{-1} . \quad{ }^{1} \mathrm{H}$ NMR (600 MHz, $\left.\mathrm{CDCl}_{3}, \mathrm{rt}\right): \delta 7.53-7.51(\mathrm{~m}, 1 \mathrm{H}), 7.48-7.45(\mathrm{~m}, 1 \mathrm{H}), 7.40-7.37(\mathrm{~m}$, $1 \mathrm{H}), 5.51(\mathrm{~d}, J=10.2 \mathrm{~Hz}, 1 \mathrm{H}), 4.19(\mathrm{q}, J=7.2 \mathrm{~Hz}, 2 \mathrm{H}), 2.35(\mathrm{dd}, J=15.0,1.8 \mathrm{~Hz}, 1 \mathrm{H}), 1.81(\mathrm{dd}, J=15.0$, $10.2 \mathrm{~Hz}, 1 \mathrm{H}), 1.39$ (s, 3H), 1.34 (s, 3H), $1.28(\mathrm{t}, J=7.2 \mathrm{~Hz}, 3 \mathrm{H}) ;{ }^{13} \mathrm{C}\left\{{ }^{1} \mathrm{H}\right\}$ NMR $\left(150 \mathrm{MHz}, \mathrm{CDCl}_{3}, \mathrm{rt}\right): \delta$ $177.3,169.2\left(\mathrm{~d},{ }^{4} J_{\mathrm{C}-\mathrm{F}}=4.7 \mathrm{~Hz}\right), 163.3\left(\mathrm{~d},{ }^{1} J_{\mathrm{C}-\mathrm{F}}=248 \mathrm{~Hz}\right), 146.1\left(\mathrm{~d},{ }^{4} J_{\mathrm{C}-\mathrm{F}}=2.4 \mathrm{~Hz}\right), 127.9\left(\mathrm{~d},{ }^{3} J_{\mathrm{C}-\mathrm{F}}=9.3\right.$ $\mathrm{Hz}), 123.8\left(\mathrm{~d},{ }^{3} J_{\mathrm{C}-\mathrm{F}}=8.1 \mathrm{~Hz}\right), 122.2\left(\mathrm{~d},{ }^{2} J_{\mathrm{C}-\mathrm{F}}=24.2 \mathrm{~Hz}\right), 112.1\left(\mathrm{~d},{ }^{2} J_{\mathrm{C}-\mathrm{F}}=23.0 \mathrm{~Hz}\right), 79.3,61.1,45.8,41.8$, 26.3, 25.3, 14.3; ${ }^{19} \mathrm{~F}\left\{{ }^{1} \mathrm{H}\right\}$ NMR (564 MHz, $\left.\mathrm{CDCl}_{3}, \mathrm{rt}\right): \delta-111.6$. HRMS (FAB) m/z: $[\mathrm{M}+\mathrm{Na}]^{+} \mathrm{Calcd}$ for $\mathrm{C}_{15} \mathrm{H}_{18} \mathrm{FO}_{4}$ 281.1189; Found: 281.1210 . 


\section{3-(2-Ethoxycarbonyl-2-methylpropyl)-5-(trifluoromethyl)phthalide (3da)}

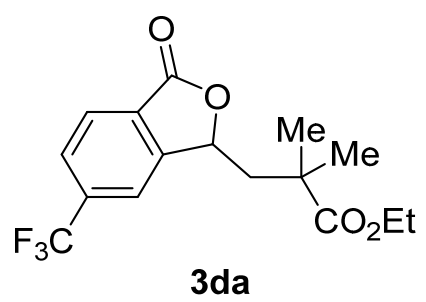

The product 3da was obtained from 4-trifruoromethyl-o-vinylbenzoic acid (1d, $84.5 \mathrm{mg}, 0.4 \mathrm{mmol})$ and ethyl $\alpha$-bromoisobutylate (2a, $156.0 \mathrm{mg}, 0.8 \mathrm{mmol}$ ) at $120^{\circ} \mathrm{C}$ for $8 \mathrm{~h}$ as a colorless oil $(57.4 \mathrm{mg}, 0.174 \mathrm{mmol}$, 43\%). $\quad R_{\mathrm{f}}=0.52($ hexane/ethyl acetate $=3 / 1) . \quad$ IR (neat): $2982(\mathrm{~m}), 1771(\mathrm{~s}), 1728(\mathrm{~s}), 1331(\mathrm{~s}), 1138(\mathrm{~s})$, $696(\mathrm{~m}) \mathrm{cm}^{-1} . \quad{ }^{1} \mathrm{H}$ NMR $\left(600 \mathrm{MHz}, \mathrm{CDCl}_{3}, \mathrm{rt}\right): \delta 8.01(\mathrm{~d}, J=8.4 \mathrm{~Hz}, 1 \mathrm{H}), 7.80(\mathrm{~d}, J=8.4 \mathrm{~Hz}, 1 \mathrm{H}), 7.78(\mathrm{~s}$, $1 \mathrm{H}), 5.61(\mathrm{~d}, J=10.2 \mathrm{~Hz}, 1 \mathrm{H}), 4.21(\mathrm{q}, J=7.2 \mathrm{~Hz}, 2 \mathrm{H}), 2.41$ (dd, $J=15.0,1.8 \mathrm{~Hz}, 1 \mathrm{H}), 1.85$ (dd, $J=15.0$, $10.2 \mathrm{~Hz}, 1 \mathrm{H}), 1.42(\mathrm{~s}, 3 \mathrm{H}), 1.36$ (s, 3H), 1.30 (t, $J=7.2 \mathrm{~Hz}, 3 \mathrm{H}) ;{ }^{13} \mathrm{C}\left\{{ }^{1} \mathrm{H}\right\}$ NMR $\left(150 \mathrm{MHz}, \mathrm{CDCl}_{3}, \mathrm{rt}\right): \delta$ $177.2,168.9,151.0,136.1\left(\mathrm{q},{ }^{2} J_{\mathrm{C}-\mathrm{F}}=32.6 \mathrm{~Hz}\right), 126.64\left(\mathrm{q},{ }^{3} J_{\mathrm{C}-\mathrm{F}}=3.5 \mathrm{~Hz}\right), 129.1,126.57,123.5\left(\mathrm{q},{ }^{1} J_{\mathrm{C}-\mathrm{F}}=\right.$ $272 \mathrm{~Hz}), 119.6$ (q, $\left.{ }^{3} J_{\mathrm{C}-\mathrm{F}}=3.5 \mathrm{~Hz}\right), 79.4,61.2,45.7,41.8,26.3,25.3,14.3 ;{ }^{19} \mathrm{~F}\left\{{ }^{1} \mathrm{H}\right\} \mathrm{NMR}\left(564 \mathrm{MHz}, \mathrm{CDCl}_{3}\right.$, rt): $\delta$-62.7. HRMS (ESI) m/z: [M $+\mathrm{Na}]^{+}$Calcd for $\mathrm{C}_{16} \mathrm{H}_{17} \mathrm{~F}_{3} \mathrm{NaO}_{4}$ 353.0977; Found: 353.0976 .

\section{6-Chloro-3-(2-ethoxycarbonyl-2-methylpropyl)phthalide (3ea)}

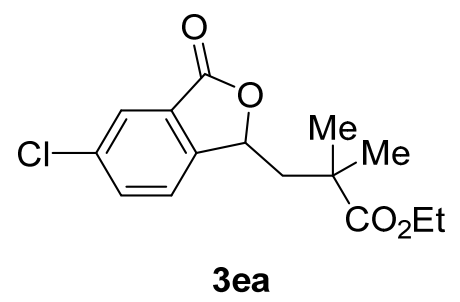

The product 3ea was obtained from 5-chloro-o-vinylbenzoic acid (1e, $73.1 \mathrm{mg}, 0.4 \mathrm{mmol})$ and ethyl $\alpha$ bromoisobutylate (2a, $156.0 \mathrm{mg}, 0.8 \mathrm{mmol})$ at $80^{\circ} \mathrm{C}$ for $16 \mathrm{~h}$ as a colorless oil $(69.7 \mathrm{mg}, 0.235 \mathrm{mmol}, 59 \%)$. $R_{\mathrm{f}}=0.55$ (hexane/ethyl acetate $\left.=3 / 1\right) . \quad$ IR (neat): $2978(\mathrm{~m}), 1771(\mathrm{~s}), 1728(\mathrm{~s}), 1472(\mathrm{~m}), 1202(\mathrm{~m}), 773$ (m) $\mathrm{cm}^{-1} . \quad{ }^{1} \mathrm{H}$ NMR $\left(600 \mathrm{MHz}, \mathrm{CDCl}_{3}, \mathrm{rt}\right): \delta 7.84(\mathrm{~d}, J=1.8 \mathrm{~Hz}, 1 \mathrm{H}), 7.64(\mathrm{dd}, J=8.4,1.8 \mathrm{~Hz}, 1 \mathrm{H}), 7.44$ (d, $J=8.4 \mathrm{~Hz}, 1 \mathrm{H}), 5.51(\mathrm{dd}, J=10.2,1.2 \mathrm{~Hz}, 1 \mathrm{H}), 4.19$ (q, $J=7.2 \mathrm{~Hz}, 2 \mathrm{H}), 2.34(\mathrm{dd}, J=15.0,1.2 \mathrm{~Hz}, 1 \mathrm{H})$, $1.80(\mathrm{dd}, J=15.0,10.2 \mathrm{~Hz}, 1 \mathrm{H}), 1.39(\mathrm{~s}, 3 \mathrm{H}), 1.34(\mathrm{~s}, 3 \mathrm{H}), 1.28(\mathrm{t}, J=7.2 \mathrm{~Hz}, 3 \mathrm{H}) ;{ }^{13} \mathrm{C}\left\{{ }^{1} \mathrm{H}\right\} \mathrm{NMR}(150$ $\left.\mathrm{MHz}, \mathrm{CDCl}_{3}, \mathrm{rt}\right): \delta 177.3,169.0,148.8,135.6,134.5,127.7,125.7,123.4,79.3,61.1,45.7,41.8,26.3,25.3$, 14.3. HRMS (FAB) m/z: [M + Na $]^{+}$Calcd for $\mathrm{C}_{15} \mathrm{H}_{18}{ }^{35} \mathrm{ClO}_{4}$ 297.0894; Found: 297.0884 . 


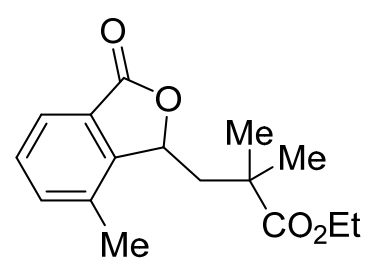

$3 f a$

The product 3fa was obtained from 3-methyl-o-vinylbenzoic acid (1f, $66.5 \mathrm{mg}, 0.4 \mathrm{mmol}$ ) and ethyl $\alpha$ bromoisobutylate (2a, $156.0 \mathrm{mg}, 0.8 \mathrm{mmol})$ at $120^{\circ} \mathrm{C}$ for $8 \mathrm{~h}$ as a colorless oil $(76.5 \mathrm{mg}, 0.277 \mathrm{mmol}, 69 \%)$. $R_{\mathrm{f}}=0.36$ (hexane/ethyl acetate = 3/1). IR (neat): $2978(\mathrm{~m}), 1769(\mathrm{~s}), 1728(\mathrm{~s}), 1476(\mathrm{~m}), 1142(\mathrm{~m}), 758(\mathrm{~m})$ $\mathrm{cm}^{-1} . \quad{ }^{1} \mathrm{H}$ NMR $\left(600 \mathrm{MHz}, \mathrm{CDCl}_{3}, \mathrm{rt}\right): \delta 7.70(\mathrm{~d}, J=6.6 \mathrm{~Hz}, 1 \mathrm{H}), 7.43-7.39(\mathrm{~m}, 2 \mathrm{H}), 5.55$ (d, $J=10.8 \mathrm{~Hz}$, 1H), 4.22-4.16 (m, 2H), 2.46 (dd, $J=15.0,1.2 \mathrm{~Hz}, 1 \mathrm{H}), 2.43$ (s, 3H), 1.66 (dd, $J=15.0,10.8 \mathrm{~Hz}, 1 \mathrm{H}$ ), 1.39 (s, 3H), 1.34 (s, 3H), $1.28(\mathrm{t}, J=7.2 \mathrm{~Hz}, 3 \mathrm{H}) ;{ }^{13} \mathrm{C}\left\{{ }^{1} \mathrm{H}\right\} \mathrm{NMR}\left(150 \mathrm{MHz}, \mathrm{CDCl}_{3}, \mathrm{rt}\right): \delta 177.4,170.7,148.7$, 135.7, 132.6, 129.4 125.9, 123.3, 79.1, 61.0, 44.1, 41.7, 26.3, 25.5, 18.1, 14.3. HRMS (ESI) m/z: [M + $\mathrm{Na}]^{+}$Calcd for $\mathrm{C}_{16} \mathrm{H}_{20} \mathrm{NaO}_{4}$ 299.1254; Found: 299.1230 .

\section{3-(2-Ethoxycarbonyl-2-methylpropyl)-6-methoxyphthalide (3ga)}

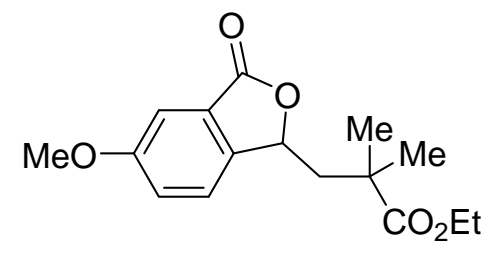

3ga

The product 3ga was obtained from 5-methoxy-o-vinylbenzoic acid (1g, $71.3 \mathrm{mg}, 0.4 \mathrm{mmol})$ and ethyl $\alpha$ bromoisobutylate (2a, $156.0 \mathrm{mg}, 0.8 \mathrm{mmol})$ at $80{ }^{\circ} \mathrm{C}$ for $16 \mathrm{~h}$ as a colorless oil $(93.5 \mathrm{mg}, 0.320 \mathrm{mmol}, 80 \%)$. $R_{\mathrm{f}}=0.31$ (hexane/ethyl acetate $\left.=3 / 1\right) . \quad$ IR (neat): $2978(\mathrm{~m}), 1763(\mathrm{~s}), 1724(\mathrm{~s}), 1497(\mathrm{~m}), 1281(\mathrm{~m}), 777$ (m) $\mathrm{cm}^{-1} . \quad{ }^{1} \mathrm{H} \mathrm{NMR}\left(600 \mathrm{MHz}, \mathrm{CDCl}_{3}, \mathrm{rt}\right): \delta 7.35(\mathrm{~d}, J=8.4 \mathrm{~Hz}, 1 \mathrm{H}), 7.30(\mathrm{~d}, J=2.4 \mathrm{~Hz}, 1 \mathrm{H}), 7.23(\mathrm{dd}, J$ $=8.4,2.4 \mathrm{~Hz}, 1 \mathrm{H}), 5.47$ (dd, $J=10.2,1.2 \mathrm{~Hz}, 1 \mathrm{H}), 4.19$ (q, $J=7.2 \mathrm{~Hz}, 2 \mathrm{H}), 3.86(\mathrm{~s}, 3 \mathrm{H}), 2.33(\mathrm{dd}, J=15.0$, $1.2 \mathrm{~Hz}, 1 \mathrm{H}), 1.79$ (dd, $J=15.0,10.2 \mathrm{~Hz}, 1 \mathrm{H}), 1.39(\mathrm{~s}, 3 \mathrm{H}), 1.34(\mathrm{~s}, 3 \mathrm{H}), 1.28(\mathrm{t}, J=7.2 \mathrm{~Hz}, 3 \mathrm{H}) ;{ }^{13} \mathrm{C}\left\{{ }^{1} \mathrm{H}\right\}$ NMR (150 MHz, $\mathrm{CDCl}_{3}$, rt): $\delta$ 177.4, 170.6, 160.8, 143.0, 127.2, 123.2, 122.9, 107.4, 79.2, 61.0, 55.9, 45.9, 41.8, 26.3, 25.3, 14.3. Calcd for $\mathrm{C}_{16} \mathrm{H}_{20} \mathrm{O}_{5}: \mathrm{C}, 65.74 ; \mathrm{H}, 6.90$. Found: $\mathrm{C}, 65.59 ; \mathrm{H}, 7.03$. 


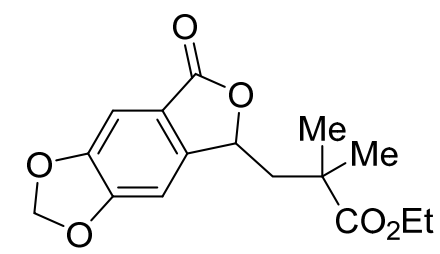

3ha

The product 3ha was obtained from 3,4-methylenedioxy-6-vinylbenzoic acid (1h, $76.9 \mathrm{mg}, 0.4 \mathrm{mmol})$ and ethyl $\alpha$-bromoisobutylate (2a, $156.0 \mathrm{mg}, 0.8 \mathrm{mmol}$ ) at $80^{\circ} \mathrm{C}$ for $16 \mathrm{~h}$ as white solid $(108.6 \mathrm{mg}, 0.355 \mathrm{mmol}$, 89\%). $\quad R_{\mathrm{f}}=0.25$ (hexane/ethyl acetate $\left.=3 / 1\right) . \quad$ M.p. $112-113^{\circ} \mathrm{C} . \quad \operatorname{IR}(\mathrm{KBr}): 2982(\mathrm{~m}), 1736(\mathrm{~s}), 1721(\mathrm{~s})$, $1481(\mathrm{~m}), 1148(\mathrm{~m}), 775(\mathrm{~m}) \mathrm{cm}^{-1}$. ${ }^{1} \mathrm{H}$ NMR (600 MHz, $\left.\mathrm{CDCl}_{3}, \mathrm{rt}\right): \delta 7.18(\mathrm{~s}, 1 \mathrm{H}), 6.84(\mathrm{~s}, 1 \mathrm{H}), 6.113$ (s, 1H), $6.111(\mathrm{~s}, 1 \mathrm{H}), 5.37$ (dd, $J=10.2,1.2 \mathrm{~Hz}, 1 \mathrm{H}), 4.19$ (q, $J=7.2 \mathrm{~Hz}, 2 \mathrm{H}), 2.29$ (dd, $J=15.0,1.2 \mathrm{~Hz}, 1 \mathrm{H}$ ), $1.76(\mathrm{dd}, J=15.0,9.6 \mathrm{~Hz}, 1 \mathrm{H}), 1.39(\mathrm{~s}, 3 \mathrm{H}), 1.32(\mathrm{~s}, 3 \mathrm{H}), 1.29(\mathrm{t}, J=7.2 \mathrm{~Hz}, 3 \mathrm{H}) ;{ }^{13} \mathrm{C}\left\{{ }^{1} \mathrm{H}\right\} \mathrm{NMR}(150 \mathrm{MHz}$, $\left.\mathrm{CDCl}_{3}, \mathrm{rt}\right): \delta 177.4,170.0,153.8,149.4,147.4,119.6,104.4,102.8,101.9,78.6,61.0,45.9,41.8,26.5,25.2$, 14.4. HRMS (FAB) $\mathrm{m} / \mathrm{z}:[\mathrm{M}+\mathrm{Na}]^{+}$Calcd for $\mathrm{C}_{16} \mathrm{H}_{19} \mathrm{O}_{6}$ 307.1182; Found: 307.1179 .

\section{1-(2-Ethoxycarbonyl-2-methylpropyl)-3-isochromanone (3ia)}

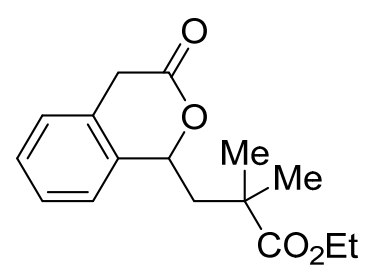

3ia

The product 3ia was obtained from 2-vinylphenylacetic acid (1i, $64.9 \mathrm{mg}, 0.4 \mathrm{mmol}$ ) and ethyl $\alpha$ bromoisobutylate (2a, $156.0 \mathrm{mg}, 0.8 \mathrm{mmol})$ at $120{ }^{\circ} \mathrm{C}$ for $8 \mathrm{~h}$ as white solid $(65.2 \mathrm{mg}, 0.236 \mathrm{mmol}, 59 \%)$. $R_{\mathrm{f}}=0.34$ (hexane/ethyl acetate =3/1). M.p. 79-80 ${ }^{\circ} \mathrm{C} . \quad \mathrm{IR}(\mathrm{KBr}): 2984(\mathrm{~m}), 1742(\mathrm{~s}), 1711$ (s), $1304(\mathrm{~m})$, $1152(\mathrm{~m}), 766(\mathrm{~m}) \mathrm{cm}^{-1} . \quad{ }^{1} \mathrm{H}$ NMR (600 MHz, $\left.\mathrm{CDCl}_{3}, \mathrm{rt}\right): \delta 7.32-7.28(\mathrm{~m}, 2 \mathrm{H}), 7.19-7.17(\mathrm{~m}, 2 \mathrm{H}), 5.48(\mathrm{dd}$, $J=11.4,3.0 \mathrm{~Hz}, 1 \mathrm{H}), 4.17-4.08(\mathrm{~m}, 2 \mathrm{H}), 3.82(\mathrm{~d}, J=18.6 \mathrm{~Hz}, 1 \mathrm{H}), 3.67$ (d, $J=18.6 \mathrm{~Hz}, 1 \mathrm{H}), 2.45$ (dd, $J=$ 14.4, $11.4 \mathrm{~Hz}, 1 \mathrm{H}), 1.87$ (dd, $J=14.4,3.0 \mathrm{~Hz}, 1 \mathrm{H}), 1.39$ (s, 3H), 1.29 (s, 3H), 1.25 (t, $J=6.6 \mathrm{~Hz}, 3 \mathrm{H}$ ); ${ }^{13} \mathrm{C}\left\{{ }^{1} \mathrm{H}\right\}$ NMR (150 MHz, $\left.\mathrm{CDCl}_{3}, \mathrm{rt}\right): \delta 177.3,170.0,134.7,130.1,128.6,127.7,127.5,124.3,79.0,61.2$, 45.4, 40.8, 35.6, 27.6, 23.8, 14.2. Calcd for $\mathrm{C}_{16} \mathrm{H}_{20} \mathrm{O}_{4}$ : C, 69.55; H, 7.30. Found: C, 69.46; H, 7.36. 


\section{1-(2-Ethoxycarbonyl-2-methylpropyl)-3,3-dimethyl-1,3-dihydroisobenzofurane (3ja)}

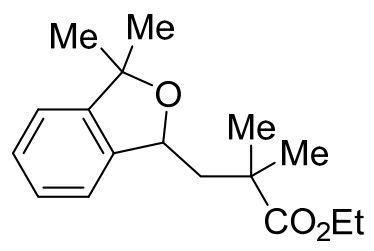

3ja

The product 3ja was obtained from 2-(2-vinylphenyl)-2-propanol (1j, $64.9 \mathrm{mg}, 0.4 \mathrm{mmol})$ and ethyl $\alpha$ bromoisobutylate (2a, $156.0 \mathrm{mg}, 0.8 \mathrm{mmol}$ ) at $80^{\circ} \mathrm{C}$ for $16 \mathrm{~h}$ as a colorless oil $(60.0 \mathrm{mg}, 0.217 \mathrm{mmol}, 54 \%)$. $R_{\mathrm{f}}=0.44$ (hexane/ethyl acetate = 8/1). IR (neat): $2974(\mathrm{~m}), 1728(\mathrm{~s}), 1474(\mathrm{~m}), 1456(\mathrm{~m}), 1138(\mathrm{~m}), 760$ (m) $\mathrm{cm}^{-1} . \quad{ }^{1} \mathrm{H}$ NMR (600 MHz, $\left.\mathrm{CDCl}_{3}, \mathrm{rt}\right): \delta$ 7.27-7.24 (m, 2H), 7.12-7.11 (m, 1H), 7.09-7.07 (m, 1H), $5.21(\mathrm{dd}, J=10.2,1.8 \mathrm{~Hz}, 1 \mathrm{H}), 4.21-4.15(\mathrm{~m}, 1 \mathrm{H}), 4.12-4.06(\mathrm{~m}, 1 \mathrm{H}), 2.08$ (dd, J = 14.4, $2.4 \mathrm{~Hz}, 1 \mathrm{H}), 1.94$ (dd, $J=14.4,9.6 \mathrm{~Hz}, 1 \mathrm{H}), 1.48(\mathrm{~s}, 3 \mathrm{H}), 1.40(\mathrm{~s}, 3 \mathrm{H}), 1.33(\mathrm{~s}, 3 \mathrm{H}), 1.32(\mathrm{~s}, 3 \mathrm{H}), 1.26(\mathrm{t}, J=7.2 \mathrm{~Hz}, 3 \mathrm{H})$; ${ }^{13} \mathrm{C}\left\{{ }^{1} \mathrm{H}\right\}$ NMR (150 MHz, $\left.\mathrm{CDCl}_{3}, \mathrm{rt}\right): \delta 178.1,147.3,142.4,127.6,127.4,121.2,120.6,85.2,78.8,60.5$, 48.5, 41.6, 30.1, 29.2, 26.3, 25.5, 14.3. Calcd for $\mathrm{C}_{17} \mathrm{H}_{24} \mathrm{O}_{3}$ : C, 73.88; H, 8.75. Found: C, 73.89; H, 9.05.

5-Chloro-1-(2-ethoxycarbonyl-2-methylpropyl)-3,3-dimethyl-1,3-dihydroisobenzofurane (3ka)

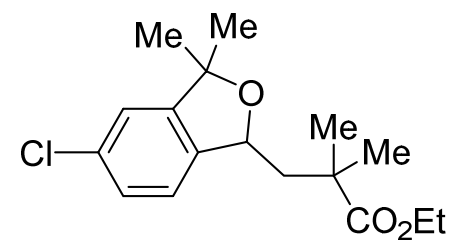

$3 k a$

The product 3ka was obtained from 2-(4-chloro-2-vinylphenyl)-2-propanol (1k $, 78.7 \mathrm{mg}, 0.4 \mathrm{mmol})$ and ethyl $\alpha$-bromoisobutylate $(2 \mathrm{a}, 156.0 \mathrm{mg}, 0.8 \mathrm{mmol})$ at $80{ }^{\circ} \mathrm{C}$ for $16 \mathrm{~h}$ as a colorless oil $(101.2 \mathrm{mg}, 0.326$ mmol, 81\%). $\quad R_{\mathrm{f}}=0.50$ (hexane/ethyl acetate $\left.=8 / 1\right) . \quad$ IR (neat): $2974(\mathrm{~m}), 1728(\mathrm{~s}), 1140(\mathrm{~m}) \mathrm{cm}^{-1} . \quad{ }^{1} \mathrm{H}$ NMR (600 MHz, $\left.\mathrm{CDCl}_{3}, \mathrm{rt}\right): \delta 7.21(\mathrm{dd}, J=7.8,1.8 \mathrm{~Hz}, 1 \mathrm{H}), 7.04-7.03(\mathrm{~m}, 2 \mathrm{H}), 5.16(\mathrm{dd}, J=10.2,1.8 \mathrm{~Hz}$, $1 \mathrm{H}), 4.20-4.14(\mathrm{~m}, 1 \mathrm{H}), 4.11-4.05(\mathrm{~m}, 1 \mathrm{H}), 2.04(\mathrm{dd}, J=14.4,1.8 \mathrm{~Hz}, 1 \mathrm{H}), 1.90(\mathrm{dd}, J=14.4,10.2 \mathrm{~Hz}, 1 \mathrm{H})$, $1.46(\mathrm{~s}, 3 \mathrm{H}), 1.38(\mathrm{~s}, 3 \mathrm{H}), 1.31(\mathrm{~s}, 3 \mathrm{H}), 1.30(\mathrm{~s}, 3 \mathrm{H}), 1.25(\mathrm{t}, J=7.2 \mathrm{~Hz}, 3 \mathrm{H}) ;{ }^{13} \mathrm{C}\left\{{ }^{1} \mathrm{H}\right\} \mathrm{NMR}(150 \mathrm{MHz}$, $\left.\mathrm{CDCl}_{3}, \mathrm{rt}\right): \delta 178.0,149.3,140.9,133.3,127.6,122.5,121.1,85.0,78.6,60.5,48.3,41.5,29.9,29.0,26.2$, 25.5, 14.3. Calcd for $\mathrm{C}_{17} \mathrm{H}_{23} \mathrm{ClO}_{3}$ : C, 65.69; $\mathrm{H}, 7.46$. Found: $\mathrm{C}, 65.82 ; \mathrm{H}, 7.55$. 


\section{Optimization of Reaction Conditions}

Ratios of Substrates ${ }^{a}$<smiles>C=Cc1ccccc1C(=O)O</smiles>

$1 a$

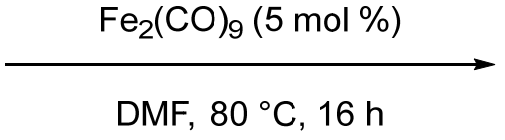

DMF, $80^{\circ} \mathrm{C}, 16 \mathrm{~h}$

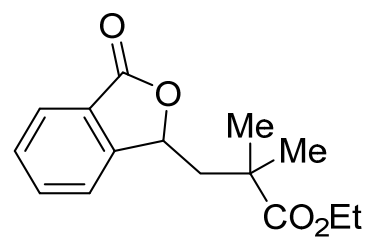

3aa

\begin{tabular}{cccc}
\hline entry & $\mathbf{1 a}(\mathrm{mmol})$ & $\mathbf{2 a}(\mathrm{mmol})$ & NMR yield $(\%)^{b}$ \\
\hline 1 & 0.4 & 0.8 & 76 \\
2 & 0.4 & 0.4 & 51 \\
3 & 0.8 & 0.4 & 59 \\
\hline
\end{tabular}

${ }^{a} 1 \mathbf{a}, 2 \mathrm{2a}$, and $\mathrm{Fe}_{2}(\mathrm{CO})_{9}(0.02 \mathrm{mmol})$ in DMF $(0.5 \mathrm{~mL})$ at $80{ }^{\circ} \mathrm{C}$ for $16 \mathrm{~h} .{ }^{b}$ Determined by ${ }^{1} \mathrm{H} \mathrm{NMR}$ analysis of the crude mixture, using dibromomethane as an internal standard.

\section{Base Screening ${ }^{a}$}<smiles>C=Cc1ccccc1C(=O)O</smiles>

$1 a$

$(0.4 \mathrm{mmol})$<smiles>CCOC(C)(C)Br</smiles>

2a

(2 equiv)

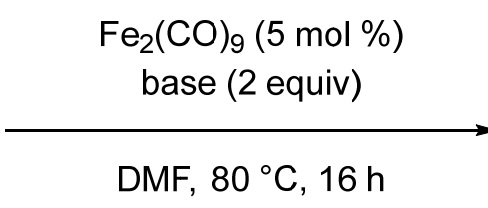

DMF, $80^{\circ} \mathrm{C}, 16 \mathrm{~h}$

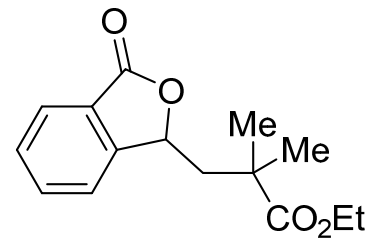

3aa

\begin{tabular}{llc}
\hline entry & base & NMR yield $(\%)^{b}$ \\
\hline 1 & none & 76 \\
2 & $\mathrm{Na}_{2} \mathrm{CO}_{3}$ & 0 \\
3 & $\mathrm{NaHCO}_{3}$ & 0 \\
4 & $\mathrm{HCOONa}$ & 6 \\
\hline
\end{tabular}

${ }^{a} 1 \mathbf{a}(0.4 \mathrm{mmol}), 2 \mathrm{a}(0.8 \mathrm{mmol})$, and $\mathrm{Fe}_{2}(\mathrm{CO})_{9}(0.02 \mathrm{mmol})$ in $\mathrm{DMF}(0.5 \mathrm{~mL})$ at $80{ }^{\circ} \mathrm{C}$ for $16 \mathrm{~h}$.

${ }^{b}$ Determined by ${ }^{1} \mathrm{H}$ NMR analysis of the crude mixture, using dibromomethane as an internal standard. 


\section{Ligand Screening $^{a}$}<smiles>C=Cc1ccccc1C(=O)O</smiles>

$1 a$<smiles>CCOC(C)(C)Br</smiles>

2a

( 2 equiv)

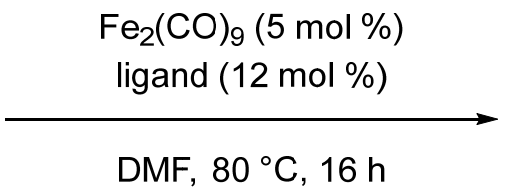

DMF, $80^{\circ} \mathrm{C}, 16 \mathrm{~h}$

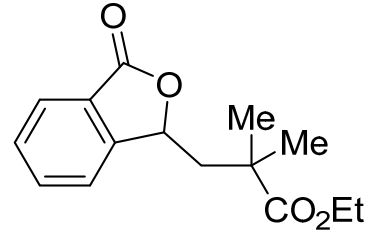

3aa

(0.4 mmol)

ligand

NMR yield (\%) ${ }^{b}$

\begin{tabular}{llc}
\hline entry & ligand & NMR yield $(\%)^{b}$ \\
\hline 1 & none & 76 \\
2 & TMEDA & 0 \\
3 & 1,10 -phenanthroline & 49 \\
4 & 2,2 '-bipyridine & 0 \\
5 & DPPP & 62 \\
6 & DPPE & 70 \\
\hline
\end{tabular}

${ }^{\mathrm{a}} \mathbf{1} \mathbf{a}(0.4 \mathrm{mmol}), \mathbf{2 a}(0.8 \mathrm{mmol})$, and $\mathrm{Fe}_{2}(\mathrm{CO})_{9}(0.02 \mathrm{mmol})$ in $\mathrm{DMF}(0.5 \mathrm{~mL})$ at $80{ }^{\circ} \mathrm{C}$ for $16 \mathrm{~h}$. ${ }^{b}$ Determined by ${ }^{1} \mathrm{H}$ NMR analysis of the crude mixture, using dibromomethane as an internal standard.<smiles>CN(C)CCN(C)C</smiles><smiles>c1cnc2c(c1)ccc1cccnc12</smiles>

TMEDA<smiles>Pc1ccccc1</smiles><smiles>Pc1ccccc1</smiles>
DPPP DPPE 


\section{Reaction Temperature ${ }^{a}$}

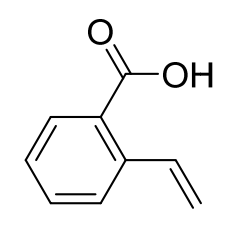

$1 \mathrm{a}$<smiles>CCOC(C)(C)Br</smiles>

$2 a$

(2 equiv)

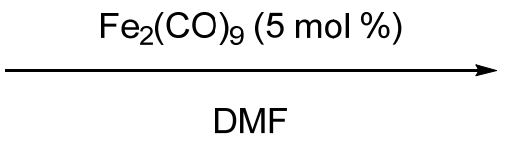

temp., $16 \mathrm{~h}$

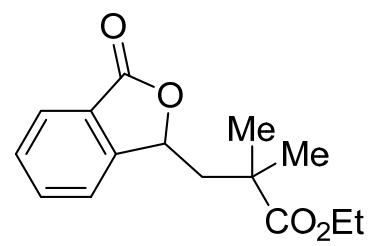

3aa

$(0.4 \mathrm{mmol})$

temp. $\left({ }^{\circ} \mathrm{C}\right)$

NMR yield $(\%)^{b}$

\begin{tabular}{lcc}
\hline entry & temp. $\left({ }^{\circ} \mathrm{C}\right)$ & NMR yield $(\%)^{b}$ \\
\hline 1 & 60 & 35 \\
2 & 70 & 63 \\
3 & 80 & 82 \\
4 & 90 & 75 \\
5 & 100 & 58 \\
\hline
\end{tabular}

${ }^{\mathrm{a}} 1 \mathrm{a}(0.4 \mathrm{mmol}), \mathbf{2 a}(0.8 \mathrm{mmol})$, and $\mathrm{Fe}_{2}(\mathrm{CO}){ }_{9}(0.02 \mathrm{mmol})$ in DMF $(0.5 \mathrm{~mL})$ for $16 \mathrm{~h} .{ }^{b}$ Determined by ${ }^{1} \mathrm{H}$ NMR analysis of the crude mixture, using dibromomethane as an internal standard. 


\section{Solvent Screening ${ }^{a}$}<smiles>C=Cc1ccccc1C(=O)O</smiles>

$1 \mathrm{a}$

$(0.4 \mathrm{mmol})$<smiles>CCOC(C)(C)Br</smiles>

2a

(2 equiv)

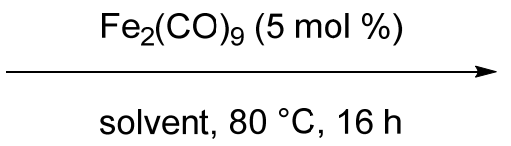

solvent, $80^{\circ} \mathrm{C}, 16 \mathrm{~h}$

\begin{tabular}{llc}
\hline entry & solvent & NMR yield $(\%)^{b}$ \\
\hline 1 & TMU & 89 \\
2 & DMF & 76 \\
3 & DMA & 33 \\
4 & DMI & 16 \\
5 & DMPU & 74 \\
6 & MeCN & 33 \\
7 & $\mathrm{NMP}$ & 83 \\
8 & MeOH & 22 \\
9 & $\mathrm{ClCH}_{2} \mathrm{CH}_{2} \mathrm{Cl}$ & 0 \\
10 & $\mathrm{CH}_{2} \mathrm{Cl}_{2}$ & 0 \\
11 & ${ }^{c}$ & 46 \\
12 & toluene & 0 \\
13 & $1,4-$ dioxane $_{2}$ & 0 \\
\hline
\end{tabular}

${ }^{a} 1 \mathbf{a}(0.4 \mathrm{mmol}), \mathbf{2 a}(0.8 \mathrm{mmol})$, and $\mathrm{Fe}_{2}(\mathrm{CO})_{9}(0.02 \mathrm{mmol})$ in solvent $(0.5 \mathrm{~mL})$ at $80{ }^{\circ} \mathrm{C}$ for $16 \mathrm{~h}$. ${ }^{b}$ Determined by ${ }^{1} \mathrm{H}$ NMR analysis of the crude mixture, using dibromomethane as an internal standard.<smiles>CN(C)C(=O)N(C)C</smiles>

TMU<smiles>CC(=O)N(C)C</smiles>

DMA<smiles>CN1CCN(C)C1=O</smiles>

DMI<smiles>CN1CCCN(C)C1=O</smiles>

DMPU<smiles>CN1CCCC1=O</smiles>

NMP 


\section{Concentrations of Substrates ${ }^{a}$}<smiles>C=Cc1ccccc1C(=O)O</smiles>

$1 \mathrm{a}$

2a

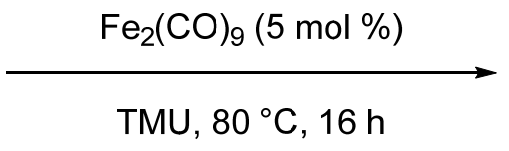

TMU, $80^{\circ} \mathrm{C}, 16 \mathrm{~h}$

( 2 equiv)

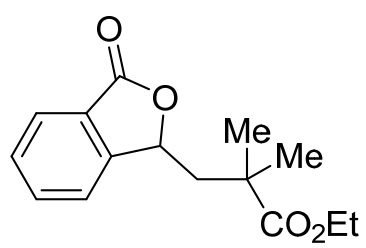

3aa

(0.4 mmol)

TMU $(\mathrm{mL})$

0.25

0.5

1
NMR yield $(\%)^{b}$

84

89

$94\left(83^{c}\right)$

${ }^{a} 1 \mathbf{a}(0.4 \mathrm{mmol}), 2 \mathrm{a}(0.8 \mathrm{mmol})$, and $\mathrm{Fe}_{2}(\mathrm{CO}) 9(0.02 \mathrm{mmol})$ in TMU at $80{ }^{\circ} \mathrm{C}$ for $16 \mathrm{~h} .{ }^{b}$ Determined by ${ }^{1} \mathrm{H}$ NMR analysis of the crude mixture, using dibromomethane as an internal standard. ${ }^{c} \mathrm{An}$ isolated yield.

\section{Catalyst Screening ${ }^{a}$}<smiles>C=Cc1ccccc1C(=O)O</smiles>

$1 \mathrm{a}$<smiles>CCOC(C)(C)Br</smiles>

2a

(2 equiv)

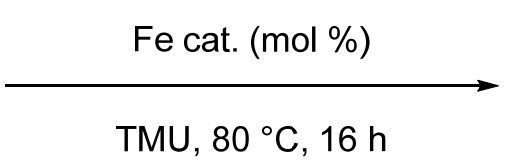

TMU, $80^{\circ} \mathrm{C}, 16 \mathrm{~h}$<smiles>CCOC(C)(C)CC1OC(=O)c2ccccc21</smiles>

3aa

(0.4 mmol)

\begin{tabular}{llc}
\hline entry & Fe cat. $(\mathrm{mol} \%)$ & NMR yield $(\%)^{b}$ \\
\hline 1 & none & 0 \\
2 & $\mathrm{Fe}_{2}(\mathrm{CO})_{9}(5)$ & $94\left(83^{c}\right)$ \\
3 & $\mathrm{Fe}(10)$ & 48 \\
4 & $\mathrm{Fe}(30)$ & 76 \\
5 & $\mathrm{FeCl}_{2}(10)$ & 82 \\
\hline
\end{tabular}

${ }^{a}$ 1a $(0.4 \mathrm{mmol}), 2 \mathrm{a}(0.8 \mathrm{mmol})$, and catalyst in TMU $(1 \mathrm{~mL})$ at $80{ }^{\circ} \mathrm{C}$ for $16 \mathrm{~h} .{ }^{b}$ Determined by ${ }^{1} \mathrm{H}$ NMR analysis of the crude mixture, using dibromomethane as an internal standard. ${ }^{c} \mathrm{An}$ isolated yield. 


\section{Substrate Limitation}

Limitation of Alkenes ${ }^{\mathrm{a}}$

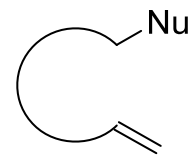

$+\mathrm{Br} \stackrel{\mathrm{Me}}{\mathrm{C}_{\mathrm{CO}_{2} \mathrm{Et}}^{\mathrm{Me}}}$
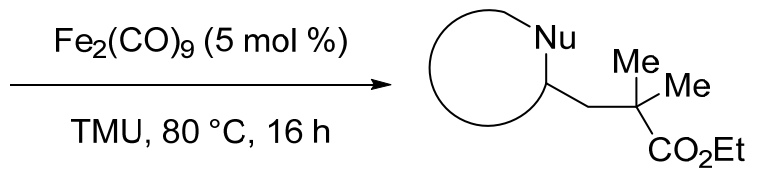

$$
\begin{aligned}
\mathrm{Nu}= & \mathrm{CO}_{2} \mathrm{H} \\
& \mathrm{CR}^{1} \mathrm{R}^{2} \mathrm{OH}
\end{aligned}
$$

1

$(0.4 \mathrm{mmol})$
$2 a$

(2 equiv)

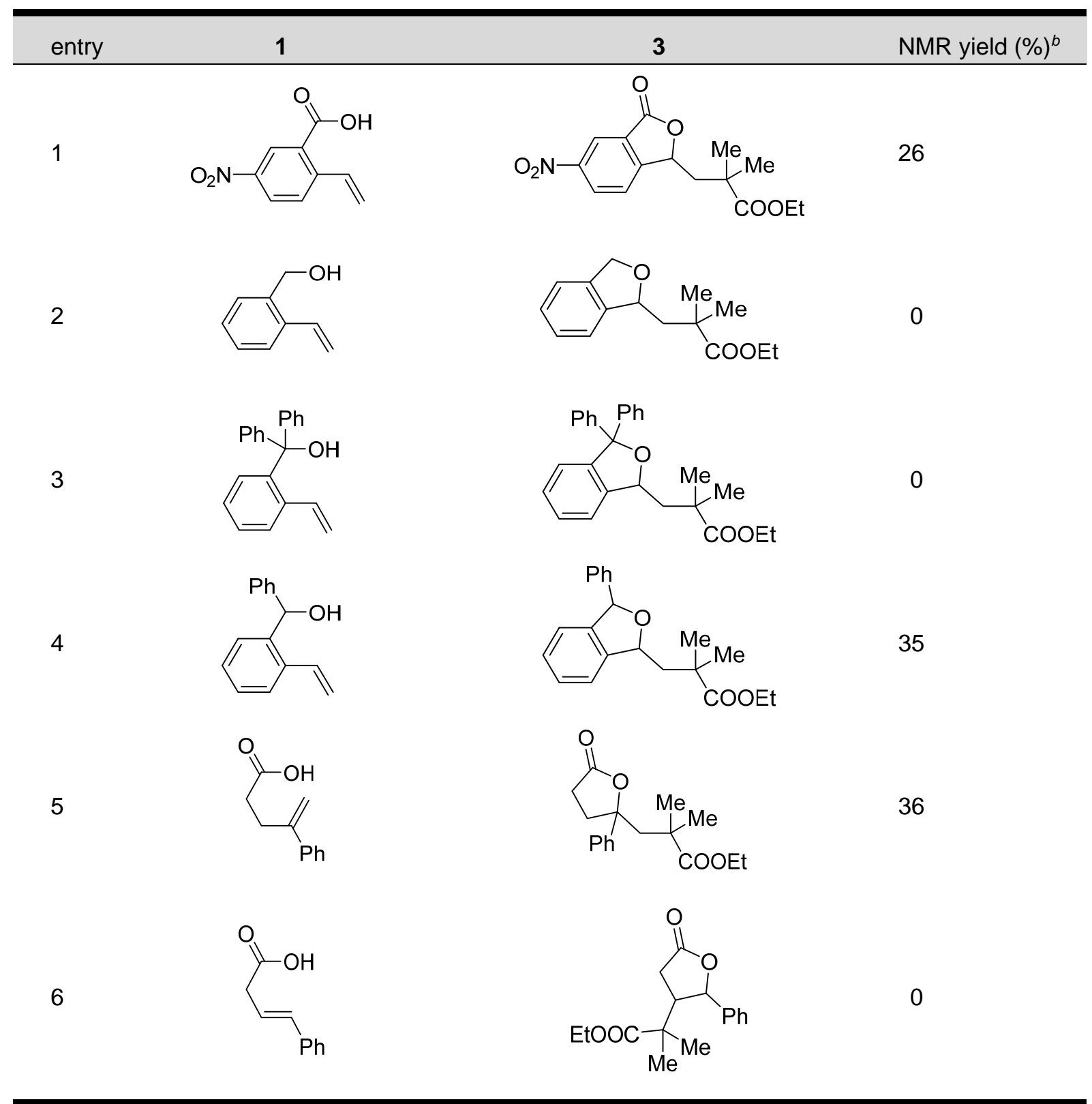

${ }^{a} 1(0.4 \mathrm{mmol}), 2 \mathrm{a}(0.8 \mathrm{mmol})$, and $\mathrm{Fe}_{2}(\mathrm{CO})_{9}(0.02 \mathrm{mmol})$ in $\mathrm{TMU}(1 \mathrm{~mL})$ at $80{ }^{\circ} \mathrm{C}$ for $16 \mathrm{~h}$. ${ }^{b}$ Determined by ${ }^{1} \mathrm{H}$ NMR analysis of the crude mixture, using dibromomethane as an internal standard. 


\section{Limitation of Alkyl Bromides ${ }^{a}$}<smiles>C=Cc1ccccc1C(=O)O</smiles>

$1 a$
$\mathrm{Br}-\mathrm{R}$

2

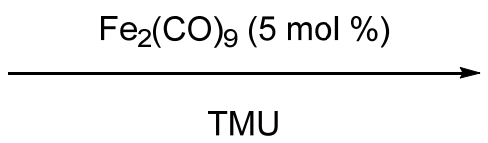

$80^{\circ} \mathrm{C}, 16 \mathrm{~h}$

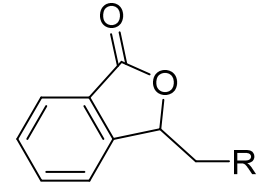

3

$(0.4 \mathrm{mmol})$ (2 equiv)

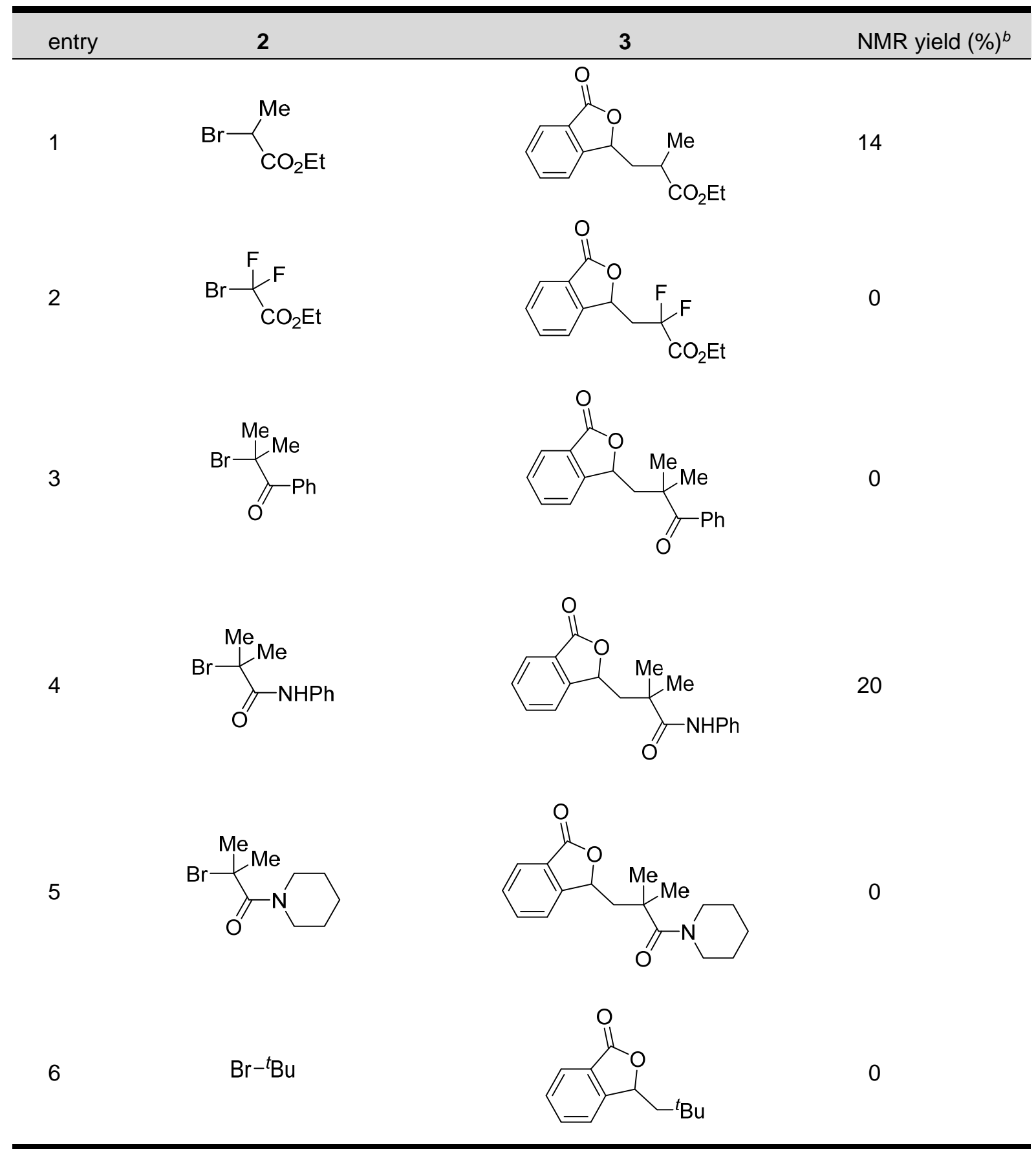

${ }^{a} 1 \mathrm{a}(0.4 \mathrm{mmol}), 2(0.8 \mathrm{mmol})$, and $\mathrm{Fe}_{2}(\mathrm{CO})_{9}(0.02 \mathrm{mmol})$ in $\mathrm{TMU}(1 \mathrm{~mL})$ at $80{ }^{\circ} \mathrm{C}$ for $16 \mathrm{~h}$. ${ }^{b}$ Determined by ${ }^{1} \mathrm{H}$ NMR analysis of the crude mixture, using dibromomethane as an internal standard. 


\section{Mechanistic Experiments}

Synthesis of 3-(2-tert-buthoxycarbonyl-2-methylpropyl)phthalide (3al) and 2-(4,4-dimethyl-5oxotetrahydrofuran-2-yl)benzoic acid (4aa) (Table 2, entry 3).<smiles>C=Cc1ccccc1C(=O)O</smiles>

$1 \mathrm{a}$

(5 mmol)<smiles>CCOC(=O)OC(C)(C)C</smiles>

21

(2 equiv)

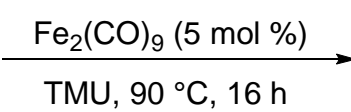

TMU, $90^{\circ} \mathrm{C}, 16 \mathrm{~h}$

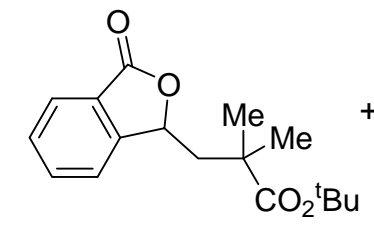

3al

$18 \%$ NMR yield $9 \%$ isolated yield

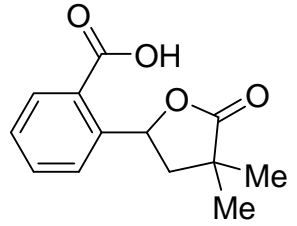

4 aa

$55 \%$ NMR yield $30 \%$ isolated yield

To a $50 \mathrm{~mL}$ Schlenk tube, were added $o$-vinylbenzoic acid (1a, $740 \mathrm{mg}, 5 \mathrm{mmol})$, tert-butyl $\alpha$ bromoisobutylate (2l, $1.86 \mathrm{ml}, 10 \mathrm{mmol}$ ), nonacarbonyldiiron (91 $\mathrm{mg}, 0.25 \mathrm{mmol}$ ) and $N, N, N^{\prime}, N^{\prime}-$ tetramethylurea (TMU, $12.5 \mathrm{~mL}$ ) under a nitrogen atmosphere. The mixture was stirred at $90{ }^{\circ} \mathrm{C}$ in a heating block for $16 \mathrm{~h}$. After the mixture was cooled to room temperature, water $(20 \mathrm{~mL})$ was added to the mixture. The mixture was extracted with ethyl acetate $(50 \mathrm{~mL})$ three times. The combined organic layers were washed with water $(50 \mathrm{~mL})$ and brine $(50 \mathrm{~mL})$. The resulting organic layer was again washed with $1 \mathrm{M}$ $\mathrm{KOH}$ aqueous solution $(50 \mathrm{~mL})$ three times. The organic layer was dried over anhydrous sodium sulfate, and volatiles were evaporated. The resulting residue was purified by silica gel column chromatography (hexane/ethyl acetate $=3 / 1)$ to provide tert-butyl 2,2-dimethyl-3-(3-oxo-1,3-dihydroisobenzofuran-1yl)propanoate (3al) as a colorless oil (126.6 mg, $0.436 \mathrm{mmol}, 9 \%) . \quad R_{\mathrm{f}}=0.52$ (hexane/ethyl acetate $=3 / 1$ ). FT-IR (neat, $\mathrm{cm}^{-1}$ ): 2976 (s), 1769 (s), 1718 (s), 1458 (m), 1282 (m), 1139 (s), 744 (m). ${ }^{1} \mathrm{H}$ NMR (600 MHz, $\left.\mathrm{CDCl}_{3}, \mathrm{rt}\right): \delta 7.89(\mathrm{~d}, J=7.8 \mathrm{~Hz}, 1 \mathrm{H}), 7.68(\mathrm{dt}, J=7.2,1.2 \mathrm{~Hz}, 1 \mathrm{H}), 7.52(\mathrm{t}, J=7.5 \mathrm{~Hz}, 1 \mathrm{H}), 7.49$ (dd, $J=$ 7.8, $0.9 \mathrm{~Hz}, 1 \mathrm{H}), 5.53(\mathrm{~d}, J=10.2 \mathrm{~Hz}, 1 \mathrm{H}), 2.34$ (dd, $J=15,1.5 \mathrm{~Hz}, 1 \mathrm{H}), 1.77$ (dd, $J=15,9.6 \mathrm{~Hz}, 1 \mathrm{H}), 1.49$ (s, 12H), 1.37 (s, 3H), $1.31(\mathrm{~s}, 3 \mathrm{H}) ;{ }^{13} \mathrm{C}\left\{{ }^{1} \mathrm{H}\right\}$ NMR (150 MHz, $\left.\mathrm{CDCl}_{3}, \mathrm{rt}\right): \delta 176.5,170.5,150.7,134.1,129.2$, 125.8, 125.7, 122.0, 80.7, 79.5, 45.7, 42.3, 28.1 (3C), 26.4, 25.4. Anal. Calcd for $\mathrm{C}_{17} \mathrm{H}_{22} \mathrm{O}_{4}$ : C, 70.32; $\mathrm{H}$, 7.64. Found: C, 70.29; H, 7.77.

After the organic layer containing 3al was removed, the combined water layers were acidified with $1 \mathrm{M}$ $\mathrm{HCl}$ aqueous solution and extracted with ethyl acetate $(50 \mathrm{~mL})$ three times. The organic layer was dried over anhydrous sodium sulfate, and volatiles were evaporated. The resulting residue was purified by bulbto-bulb distillation $\left(110^{\circ} \mathrm{C}, 3-4\right.$ bar) and the remained oily product was purified by silica gel column chromatography (hexane/ethyl acetate $=2 / 1)$ to provide 2-(4,4-dimethyl-5-oxotetrahydrofuran-2-yl)benzoic acid (4aa) as a white solid (353.7 mg, $1.51 \mathrm{mmol}, 30 \%) . \quad R_{\mathrm{f}}=0.24$ (hexane/ethyl acetate $\left.=2 / 1\right) . \quad$ Mp 171$173{ }^{\circ} \mathrm{C} . \quad$ FT-IR (KBr, cm $\left.{ }^{-1}\right): 3057$ (br), 2970 (m) 1770 (s), 1697 (s), 1411 (m), 1300 (m), 1153 (m), 925 (m). 
${ }^{1} \mathrm{H}$ NMR (600 MHz, $\left.\mathrm{CDCl}_{3}, \mathrm{rt}\right): \delta 8.16(\mathrm{dd}, J=6.6,1.2 \mathrm{~Hz}, 1 \mathrm{H}), 7.71(\mathrm{~d}, J=7.8 \mathrm{~Hz}, 1 \mathrm{H}), 7.66$ (dd, $J=7.8$, $1.2 \mathrm{~Hz}, 1 \mathrm{H}), 7.44$ (dd, $J=7.2,1.2 \mathrm{~Hz}, 1 \mathrm{H}), 6.32(\mathrm{dd}, J=7.2,1.8 \mathrm{~Hz}, 1 \mathrm{H}), 2.84(\mathrm{dd}, J=13.2,7.2 \mathrm{~Hz}, 1 \mathrm{H})$, $1.90(\mathrm{dd}, J=13.2,9.0 \mathrm{~Hz}, 1 \mathrm{H}), 1.40(\mathrm{~s}, 3 \mathrm{H}), 1.27(\mathrm{~s}, 3 \mathrm{H}) ;{ }^{13} \mathrm{C}\left\{{ }^{1} \mathrm{H}\right\} \mathrm{NMR}\left(150 \mathrm{MHz}, \mathrm{CDCl}_{3}, \mathrm{rt}\right): \delta 182.3$, 171.1, 143.7, 134.3, 132.1, 128.0, 125.7, 125.5, 75.9, 46.1, 40.6, 25.4, 24.7. Anal. Calcd for $\mathrm{C}_{13} \mathrm{H}_{14} \mathrm{O}_{4}$ : C, 66.66; H, 6.02. Found: C, 66.72; H, 6.02.

The NMR yield of 3al and 4aa were calculated to be $18 \%$ and $55 \%$, respectively. 


\section{Copies of ${ }^{1} \mathrm{H},{ }^{13} \mathrm{C}\left\{{ }^{1} \mathrm{H}\right\}$, and ${ }^{19} \mathrm{~F}\left\{{ }^{1} \mathrm{H}\right\}$ NMR Charts for the New Compounds}
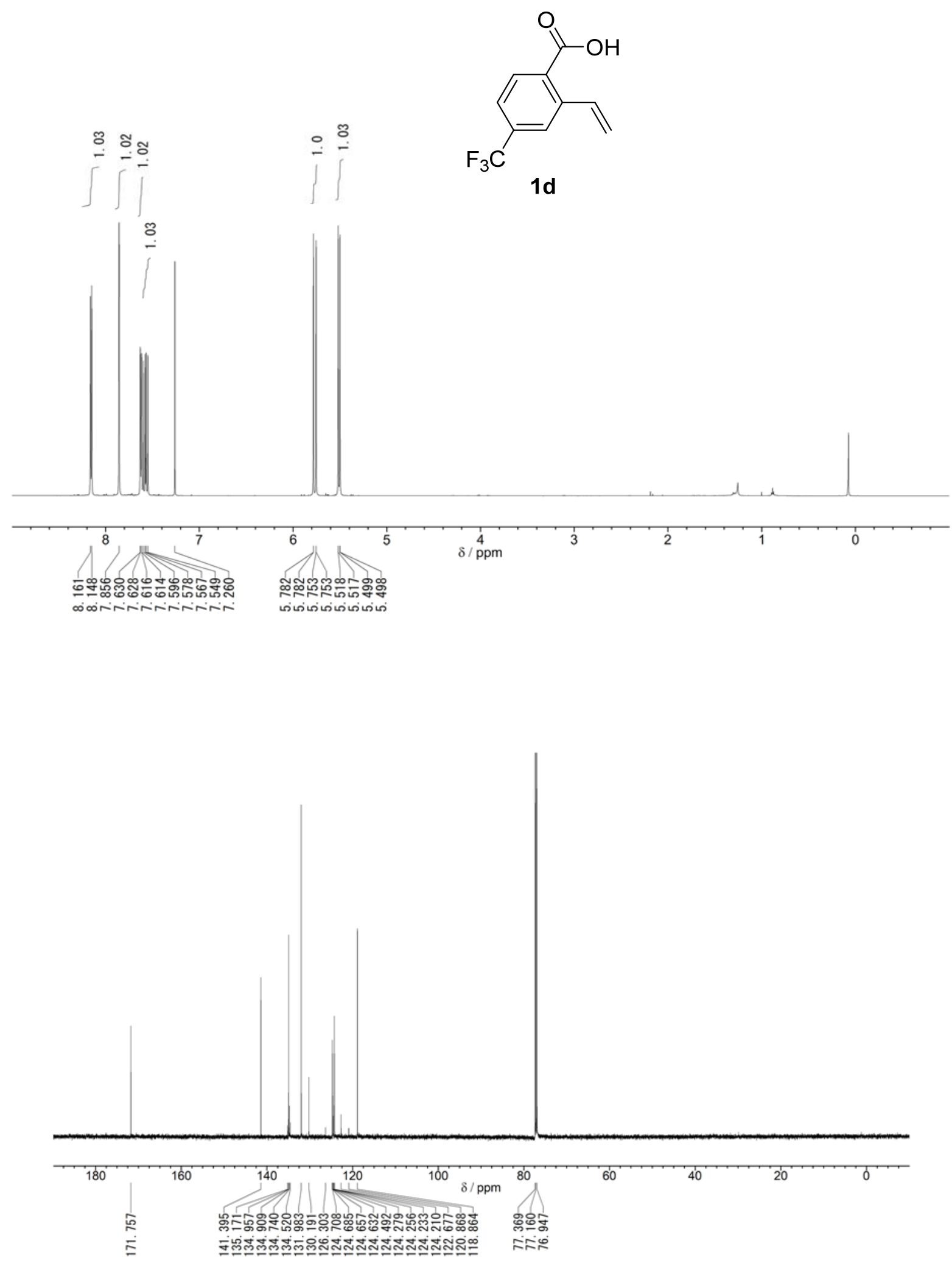

${ }^{1} \mathrm{H}$ NMR $(600 \mathrm{MHz})$ and ${ }^{13} \mathrm{C}\left\{{ }^{1} \mathrm{H}\right\}$ NMR $(150 \mathrm{MHz})$ spectra of $\mathbf{1 d}\left(\mathrm{CDCl}_{3}, \mathrm{rt}\right)$. 


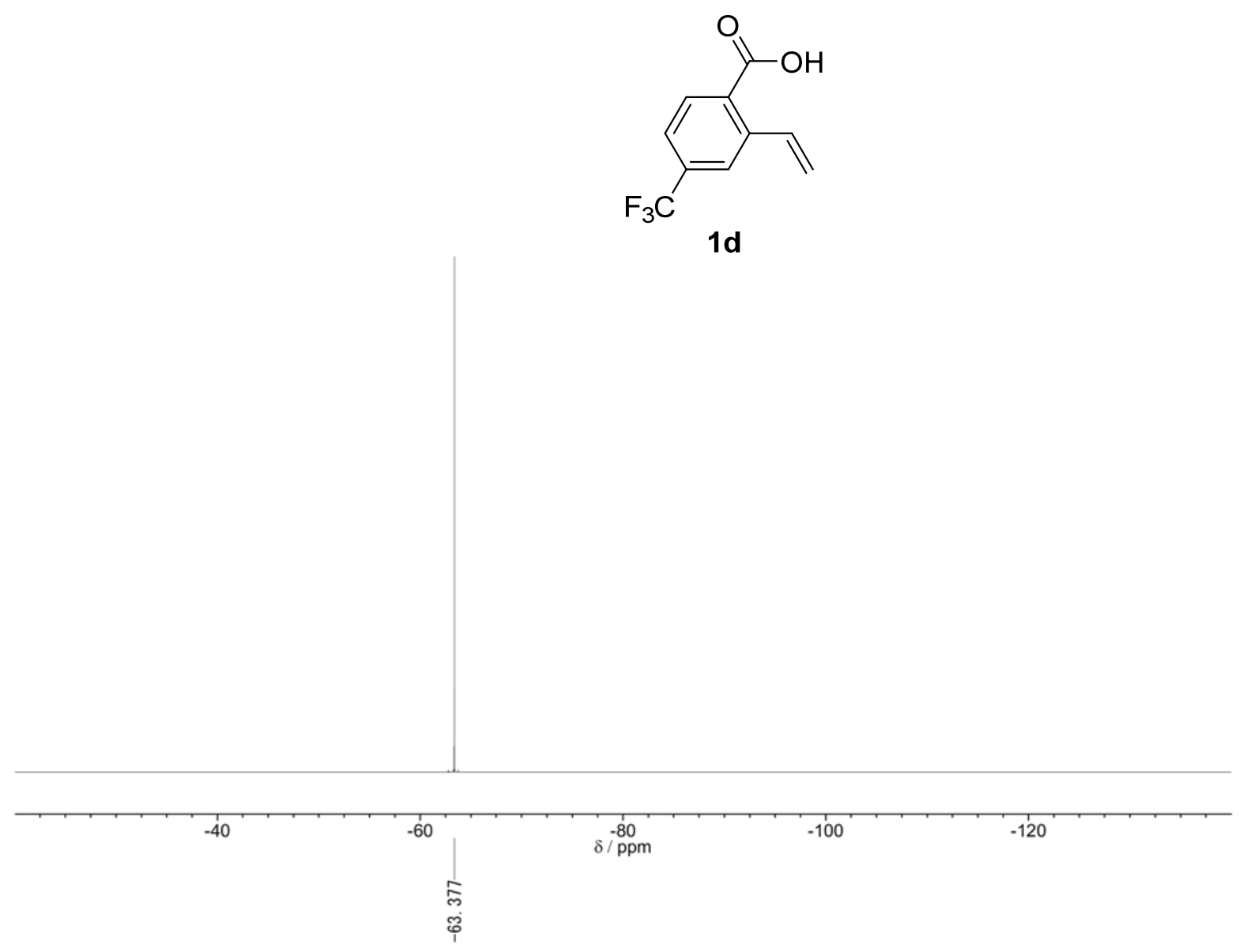

${ }^{19} \mathrm{~F}\left\{{ }^{1} \mathrm{H}\right\}$ NMR (564 MHz) spectra of $\mathbf{1 d}\left(\mathrm{CDCl}_{3}, \mathrm{rt}\right)$. 


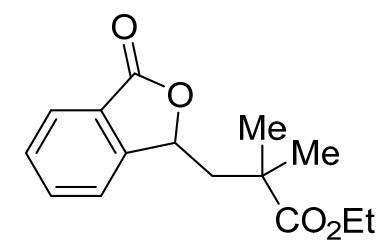

3aa
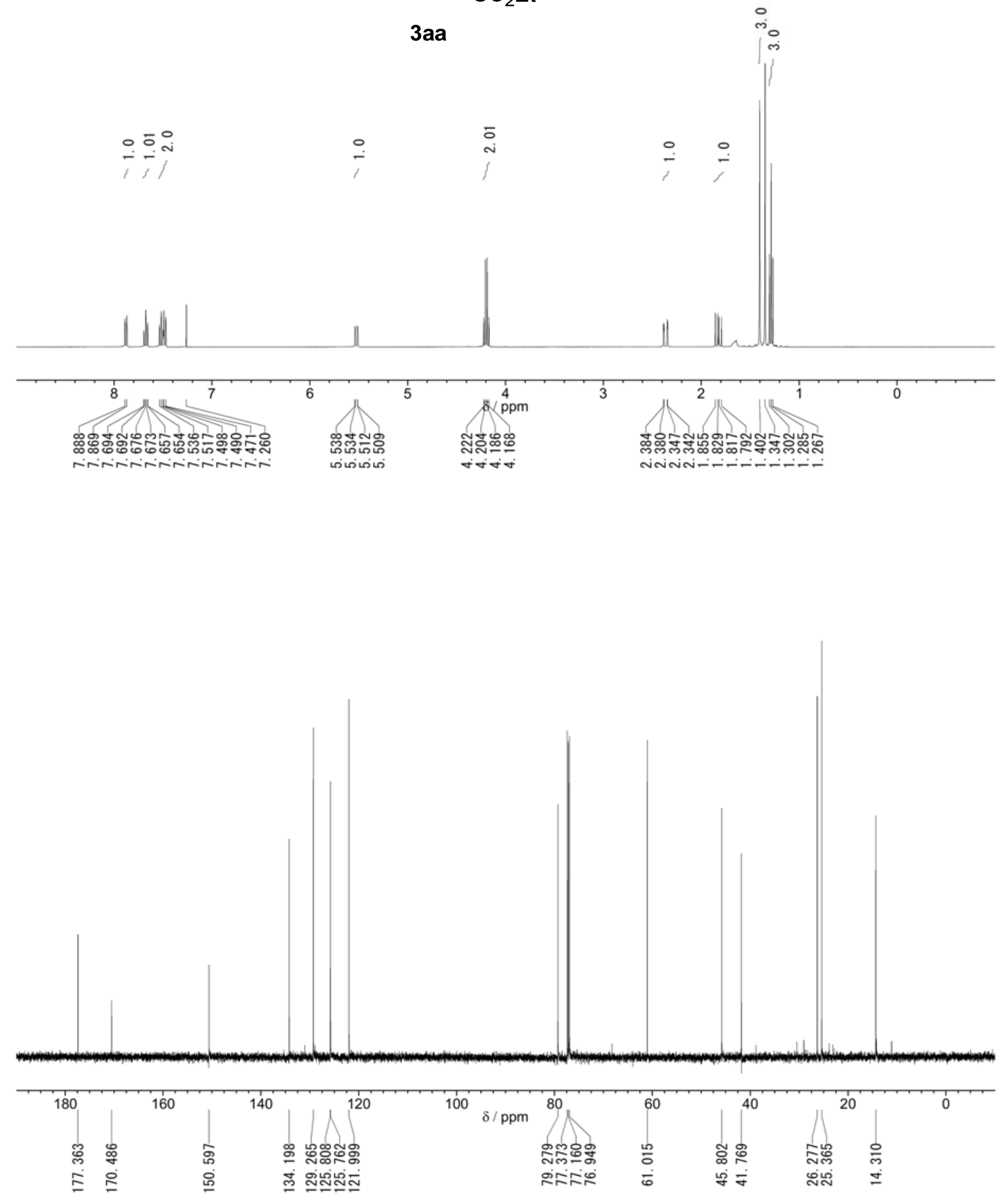

${ }^{1} \mathrm{H}$ NMR $(400 \mathrm{MHz})$ and ${ }^{13} \mathrm{C}\left\{{ }^{1} \mathrm{H}\right\}$ NMR $(150 \mathrm{MHz})$ spectra of 3aa $\left(\mathrm{CDCl}_{3}, \mathrm{rt}\right)$. 

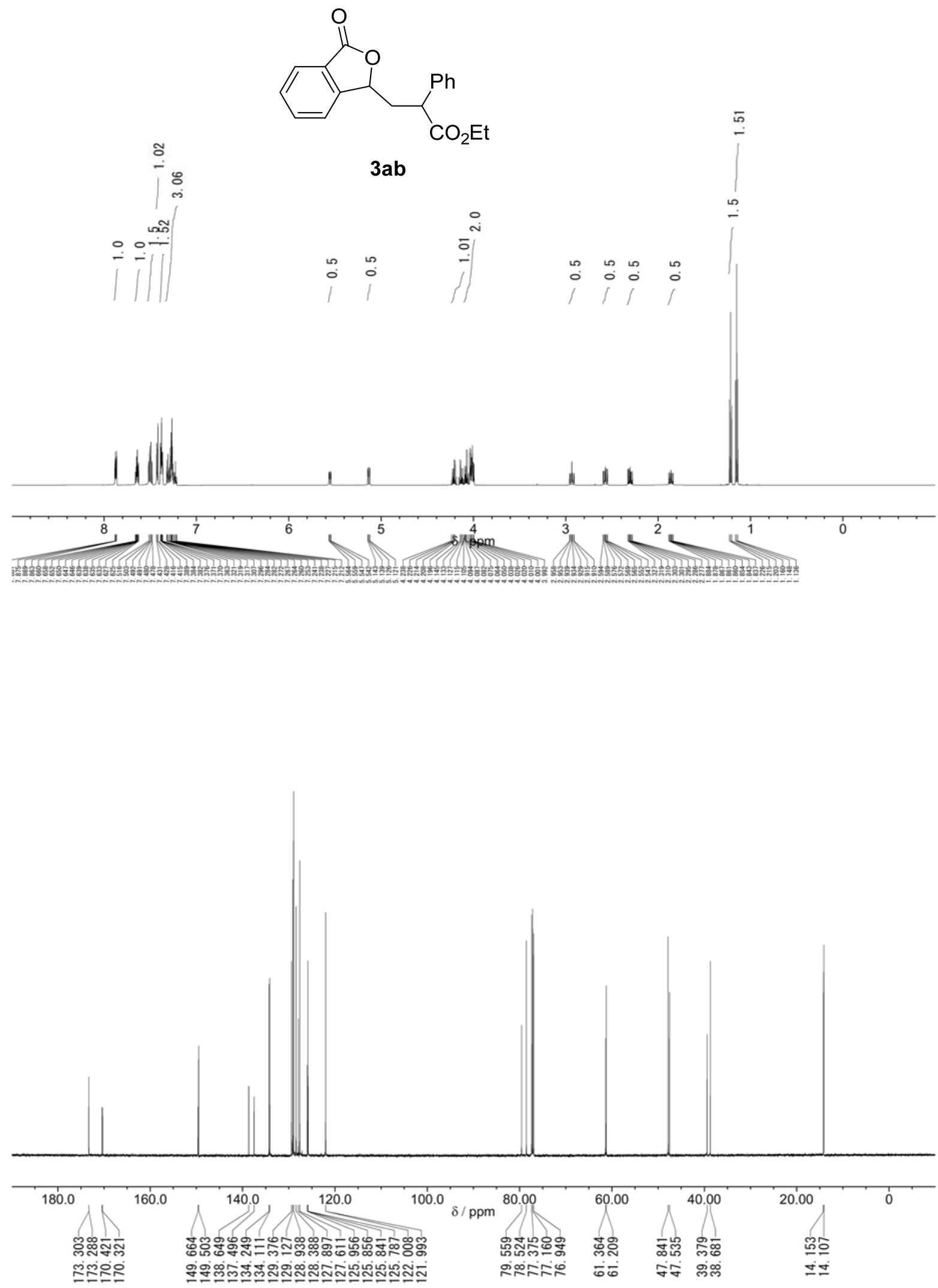

${ }^{1} \mathrm{H}$ NMR $(600 \mathrm{MHz})$ and ${ }^{13} \mathrm{C}\left\{{ }^{1} \mathrm{H}\right\}$ NMR $(150 \mathrm{MHz})$ spectra of $\mathbf{3 a b}\left(\mathrm{CDCl}_{3}, \mathrm{rt}\right)$. 

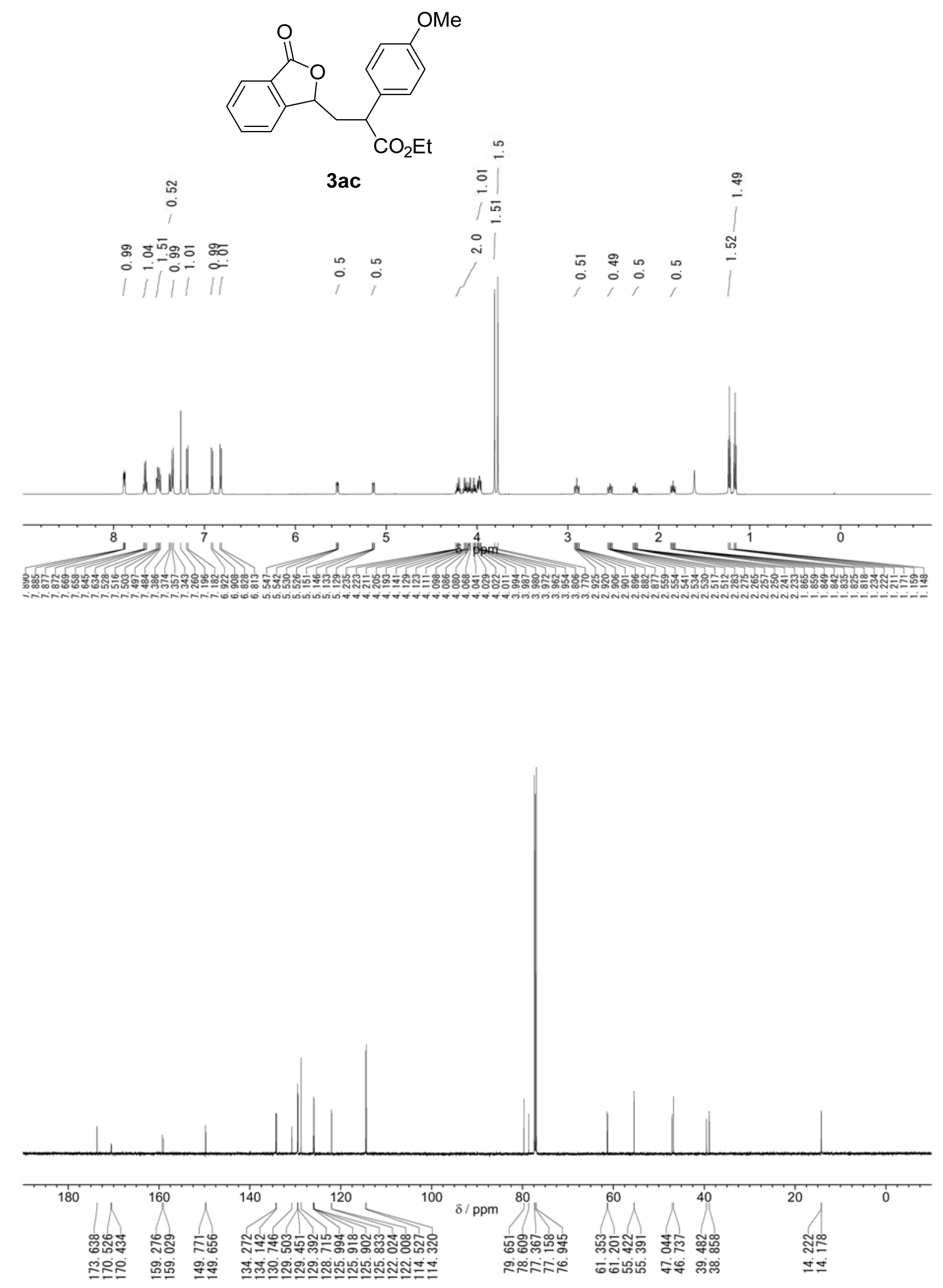

${ }^{1} \mathrm{H}$ NMR $(600 \mathrm{MHz})$ and ${ }^{13} \mathrm{C}\left\{{ }^{1} \mathrm{H}\right\}$ NMR $(150 \mathrm{MHz})$ spectra of 3ac $\left(\mathrm{CDCl}_{3}, \mathrm{rt}\right)$. 

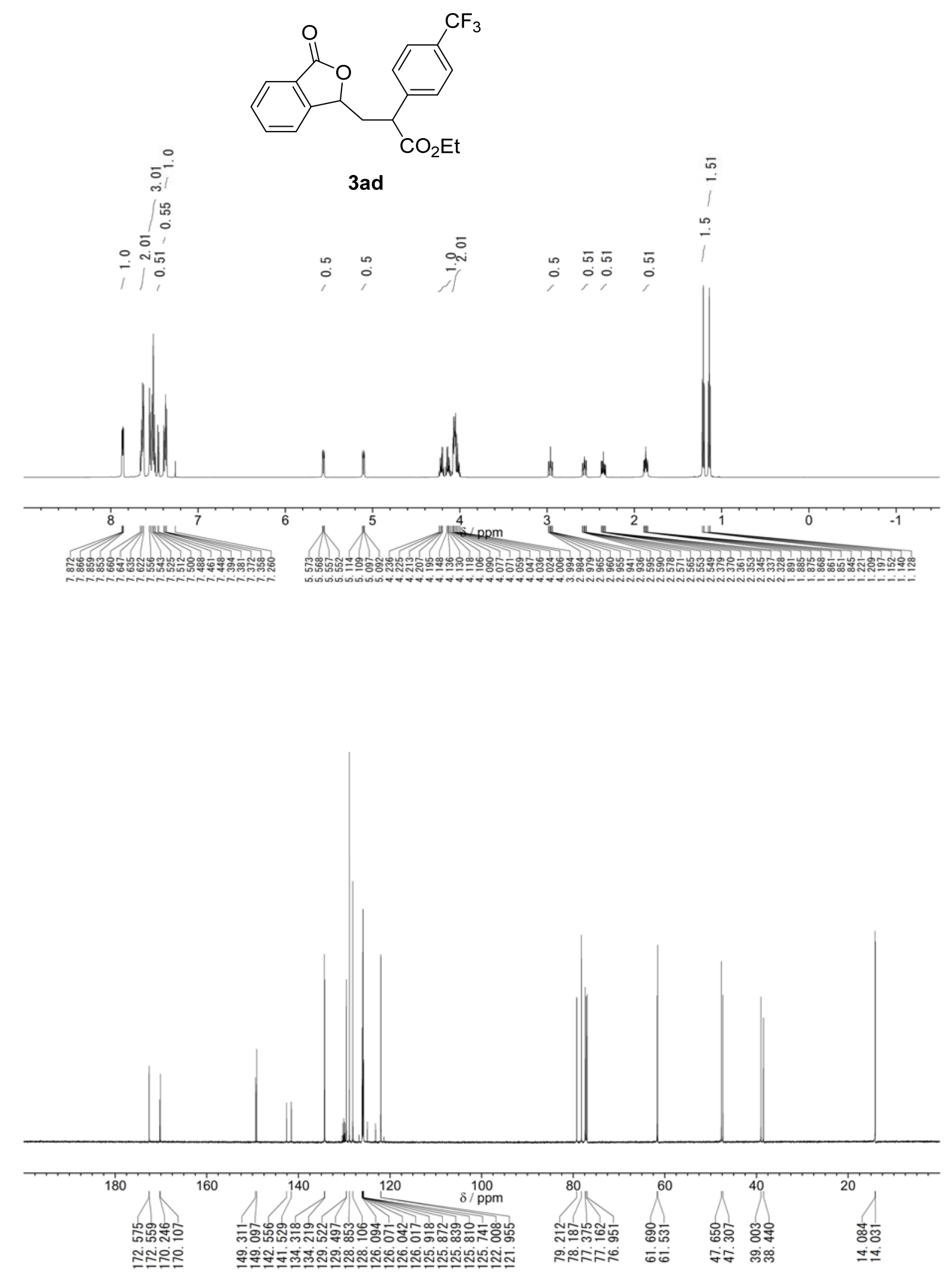

${ }^{1} \mathrm{H}$ NMR $(600 \mathrm{MHz})$ and ${ }^{13} \mathrm{C}\left\{{ }^{1} \mathrm{H}\right\}$ NMR $(150 \mathrm{MHz})$ spectra of 3ad $\left(\mathrm{CDCl}_{3}, \mathrm{rt}\right)$. 


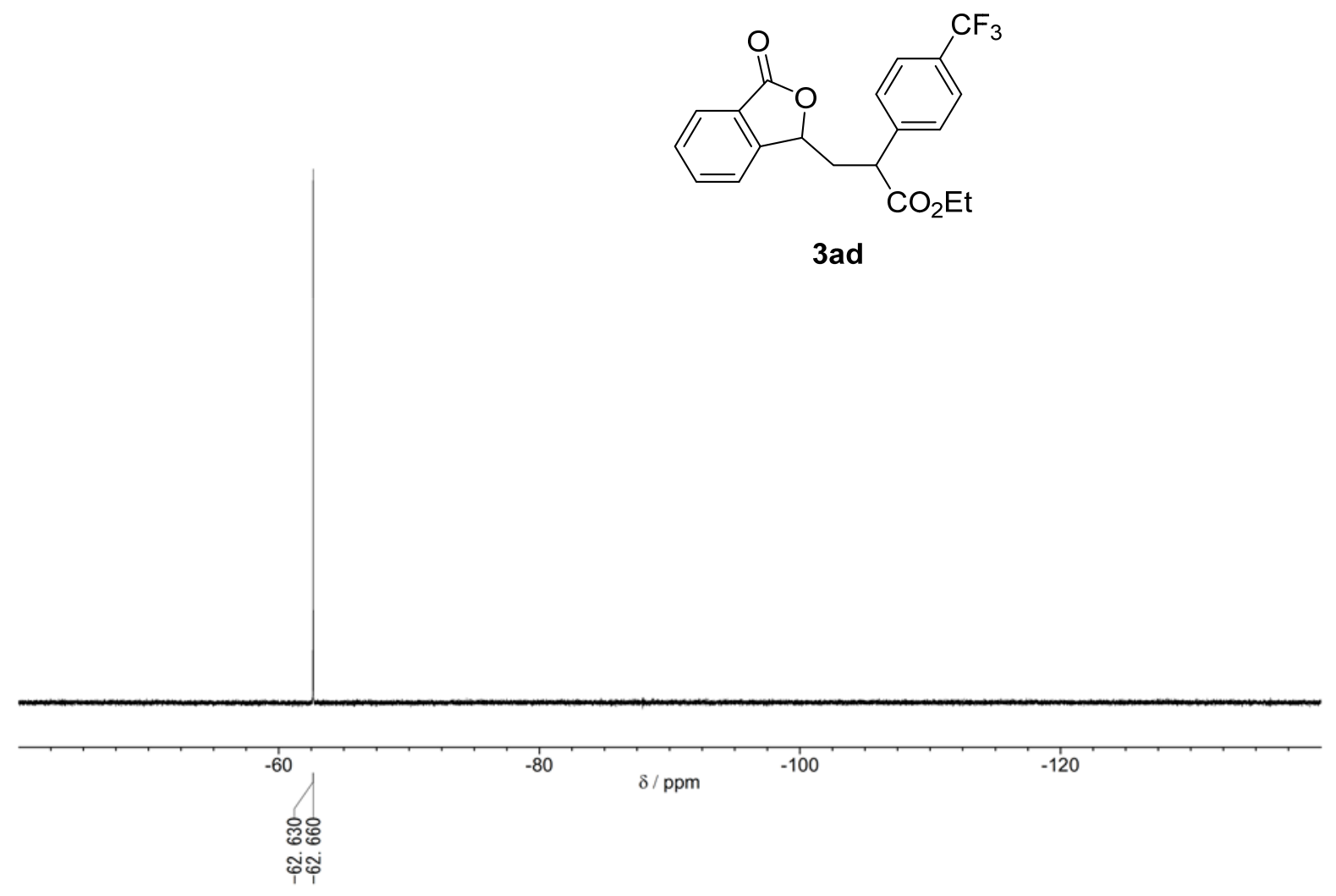

${ }^{19} \mathrm{~F}\left\{{ }^{1} \mathrm{H}\right\}$ NMR (564 MHz) spectra of 3ad $\left(\mathrm{CDCl}_{3}, \mathrm{rt}\right)$. 

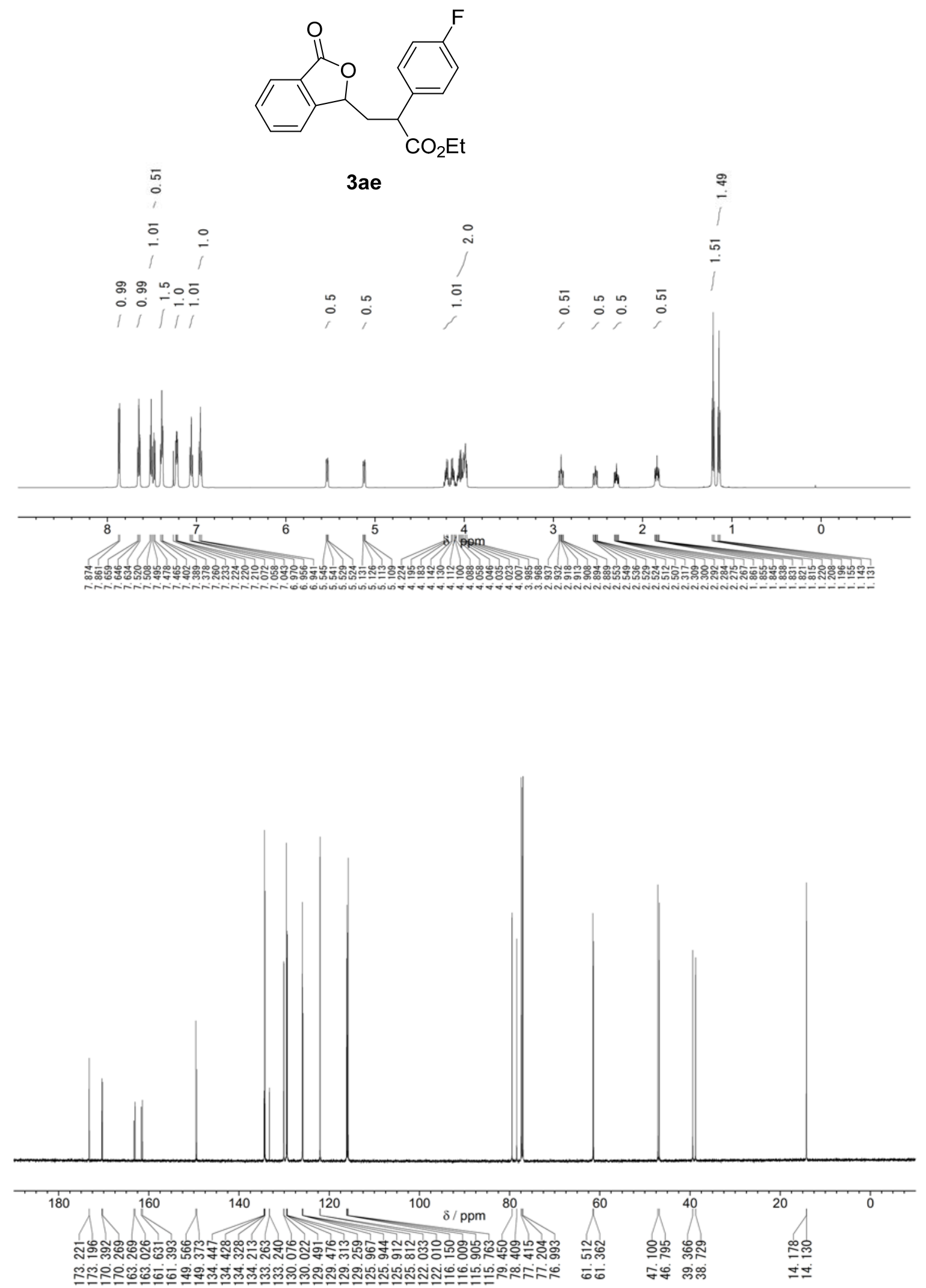

${ }^{1} \mathrm{H}$ NMR $(600 \mathrm{MHz})$ and ${ }^{13} \mathrm{C}\left\{{ }^{1} \mathrm{H}\right\}$ NMR $(150 \mathrm{MHz})$ spectra of 3ae $\left(\mathrm{CDCl}_{3}, \mathrm{rt}\right)$. 


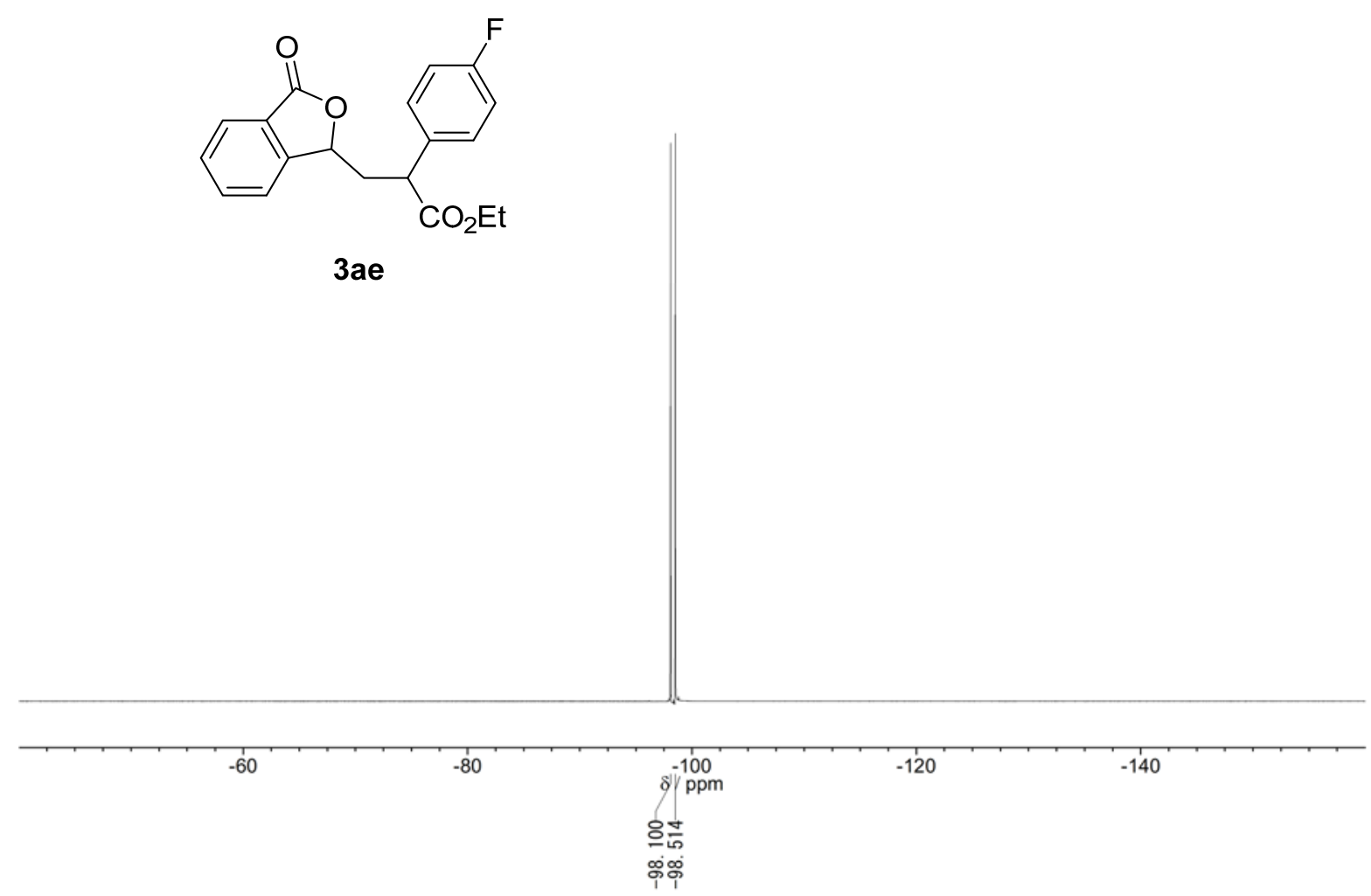

${ }^{19} \mathrm{~F}\left\{{ }^{1} \mathrm{H}\right\}$ NMR $(564 \mathrm{MHz})$ spectra of 3ae $\left(\mathrm{CDCl}_{3}, \mathrm{rt}\right)$. 

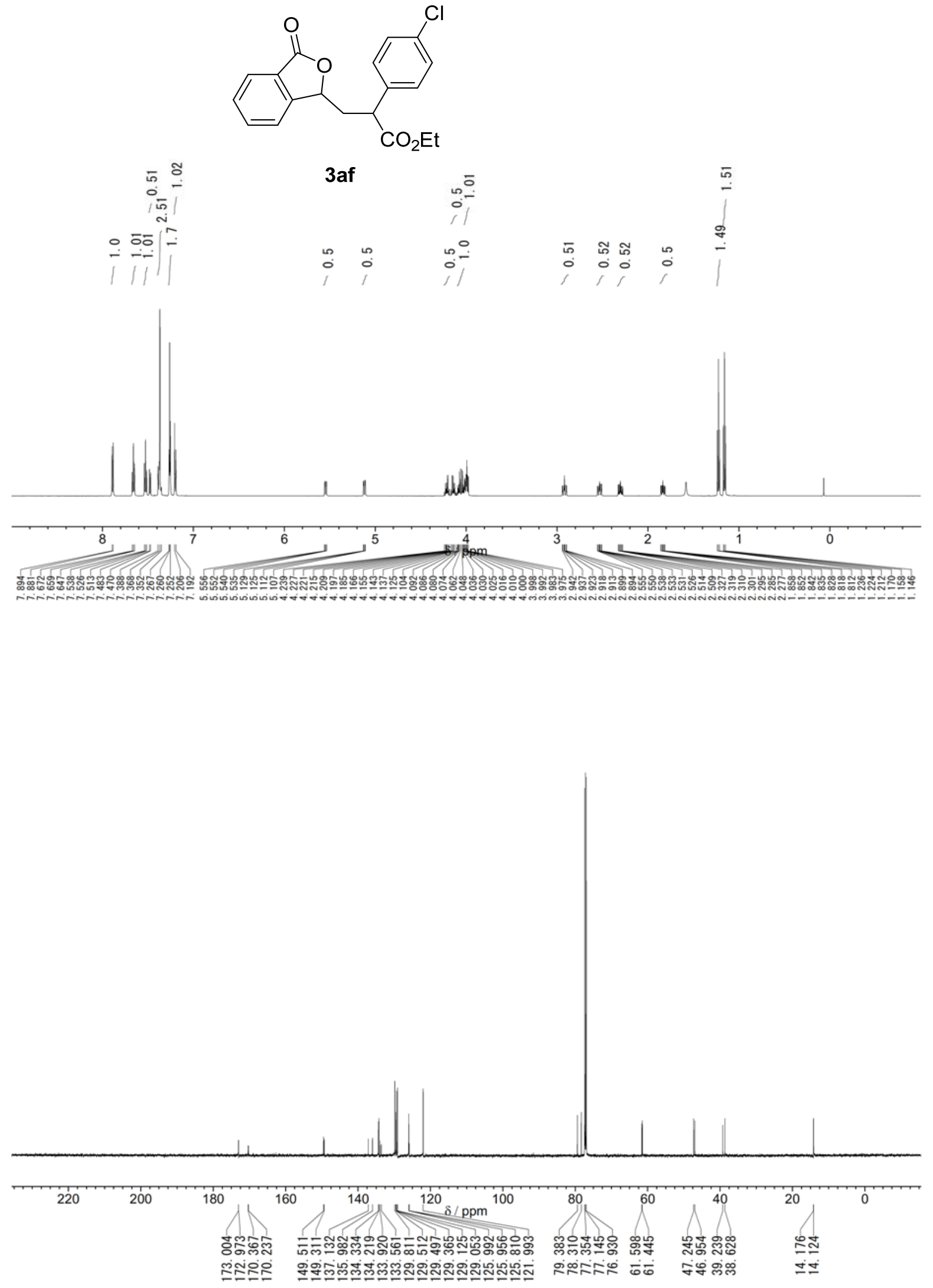

${ }^{1} \mathrm{H}$ NMR $(600 \mathrm{MHz})$ and ${ }^{13} \mathrm{C}\left\{{ }^{1} \mathrm{H}\right\}$ NMR $(150 \mathrm{MHz})$ spectra of 3 af $\left(\mathrm{CDCl}_{3}, \mathrm{rt}\right)$. 
<smiles>CCOC(=O)C1(CC2OC(=O)c3ccccc32)CCC1</smiles>

3ag
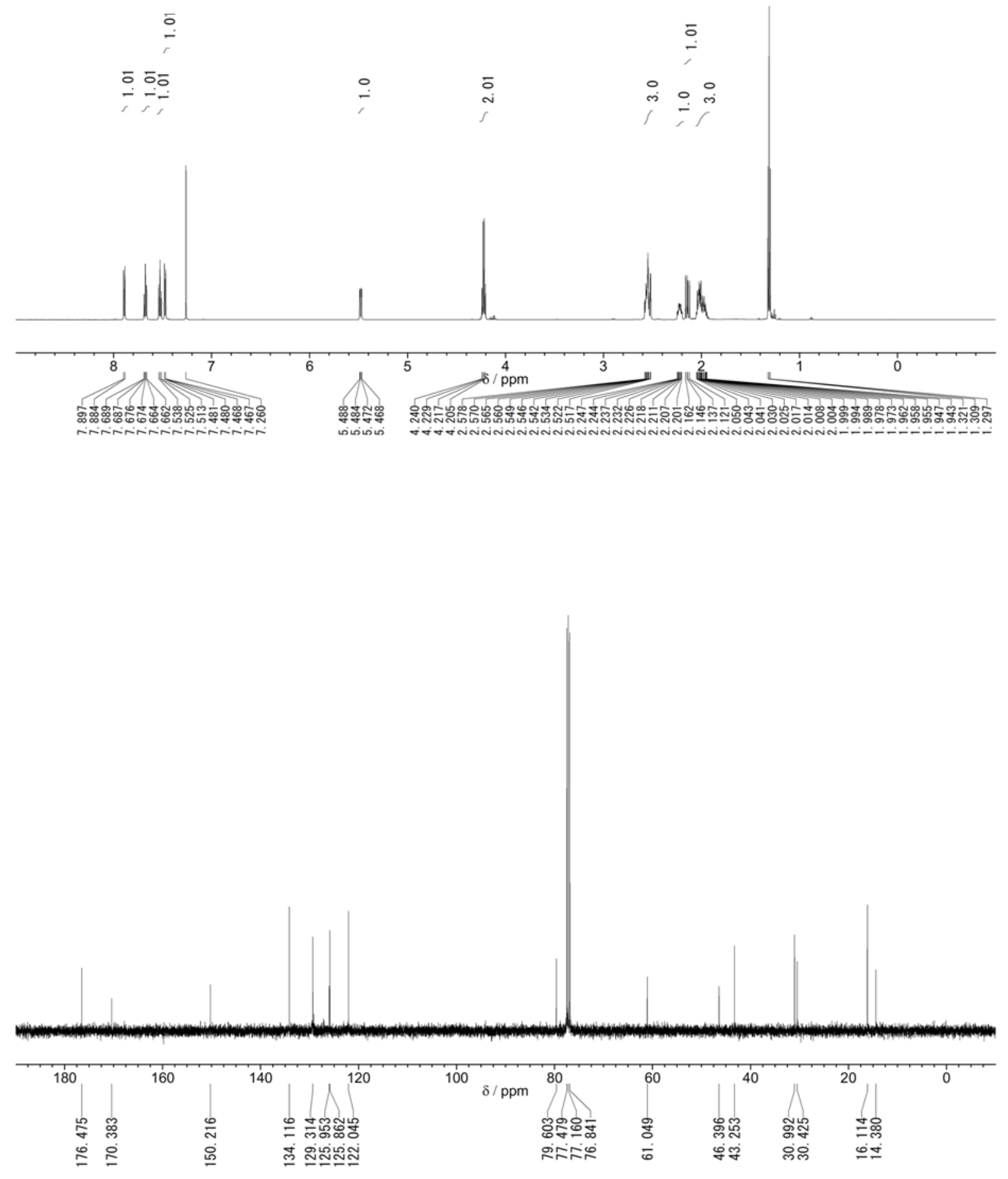

${ }^{1} \mathrm{H}$ NMR $(600 \mathrm{MHz})$ and ${ }^{13} \mathrm{C}\left\{{ }^{1} \mathrm{H}\right\}$ NMR $(100 \mathrm{MHz})$ spectra of $\mathbf{3 a g}\left(\mathrm{CDCl}_{3}, \mathrm{rt}\right)$. 

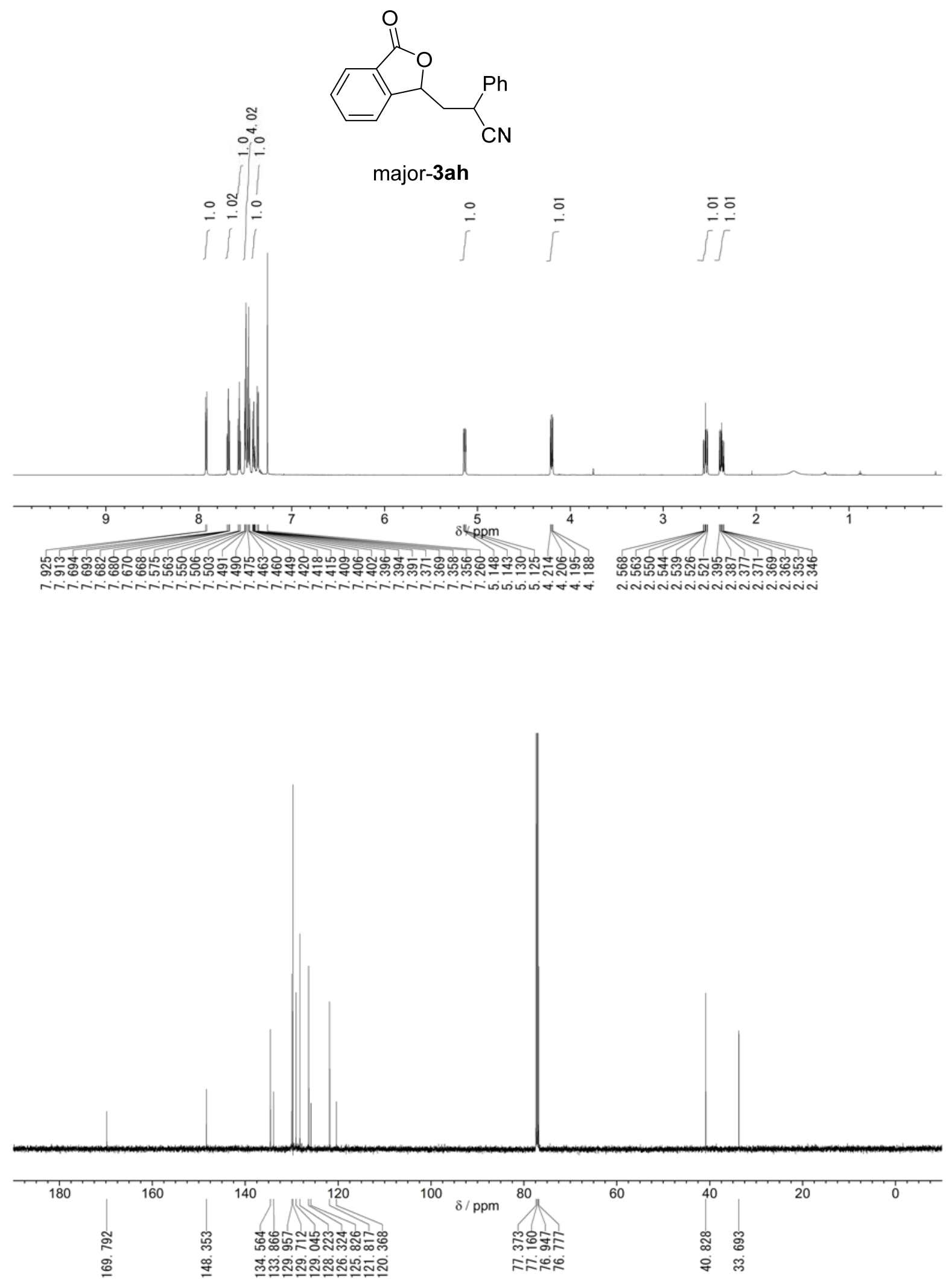

${ }^{1} \mathrm{H}$ NMR $(600 \mathrm{MHz})$ and ${ }^{13} \mathrm{C}\left\{{ }^{1} \mathrm{H}\right\}$ NMR $(150 \mathrm{MHz})$ spectra of major-3ah $\left(\mathrm{CDCl}_{3}, \mathrm{rt}\right)$. 

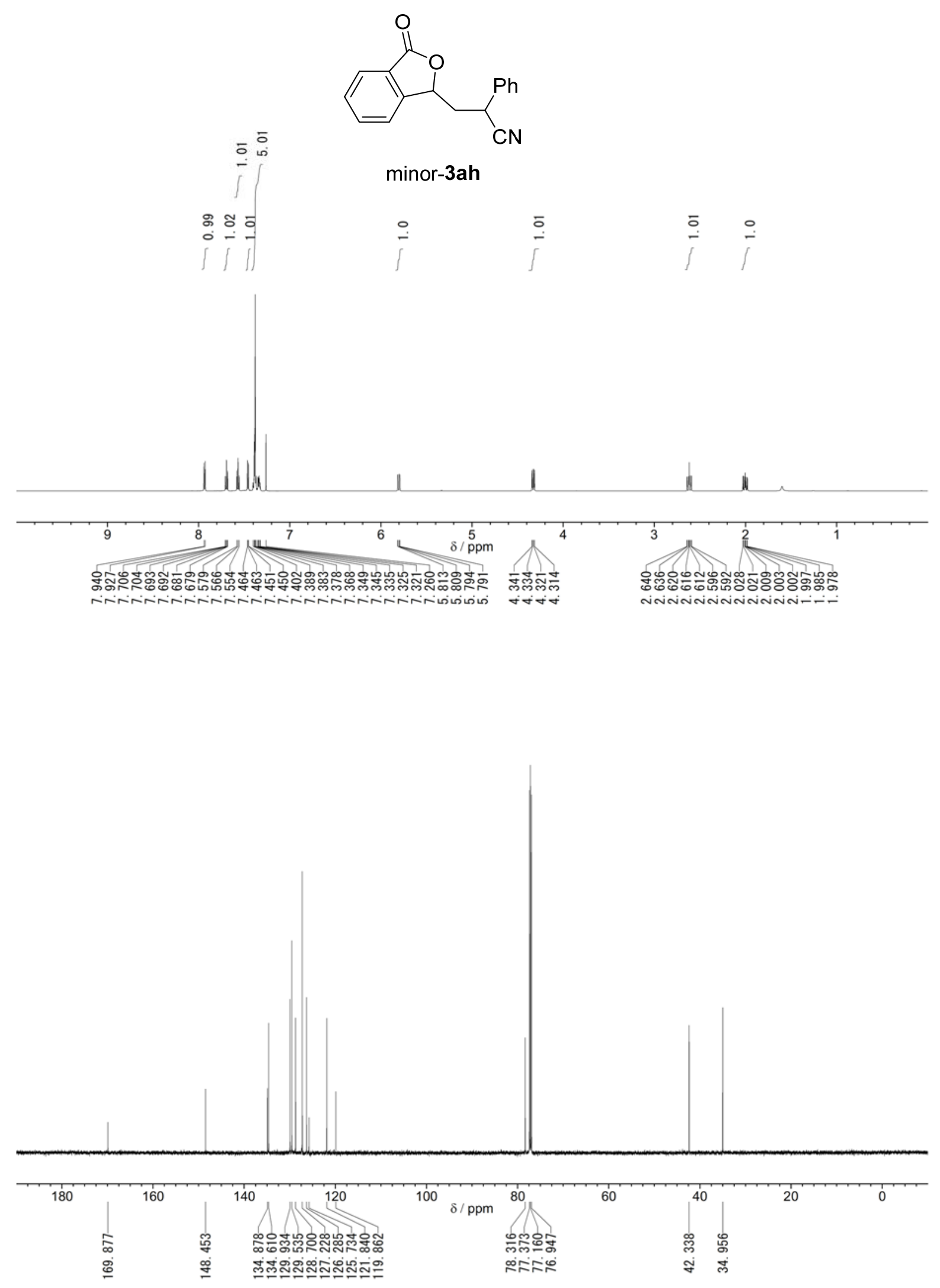

${ }^{1} \mathrm{H}$ NMR (600 MHz) and ${ }^{13} \mathrm{C}\left\{{ }^{1} \mathrm{H}\right\}$ NMR (150 MHz) spectra of minor-3ah $\left(\mathrm{CDCl}_{3}, \mathrm{rt}\right)$. 

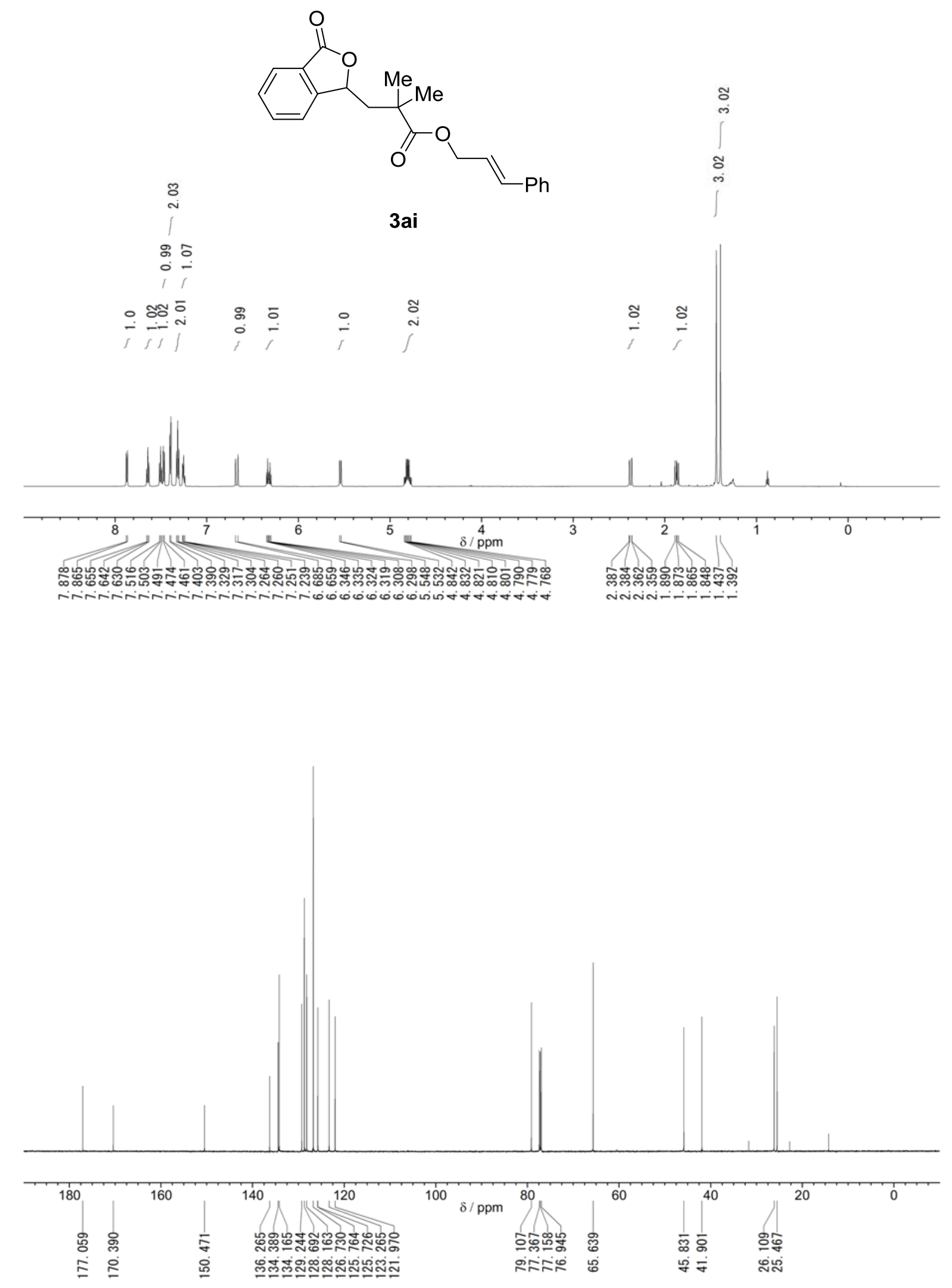

${ }^{1} \mathrm{H}$ NMR $(600 \mathrm{MHz})$ and ${ }^{13} \mathrm{C}\left\{{ }^{1} \mathrm{H}\right\}$ NMR $(150 \mathrm{MHz})$ spectra of 3ai $\left(\mathrm{CDCl}_{3}, \mathrm{rt}\right)$. 

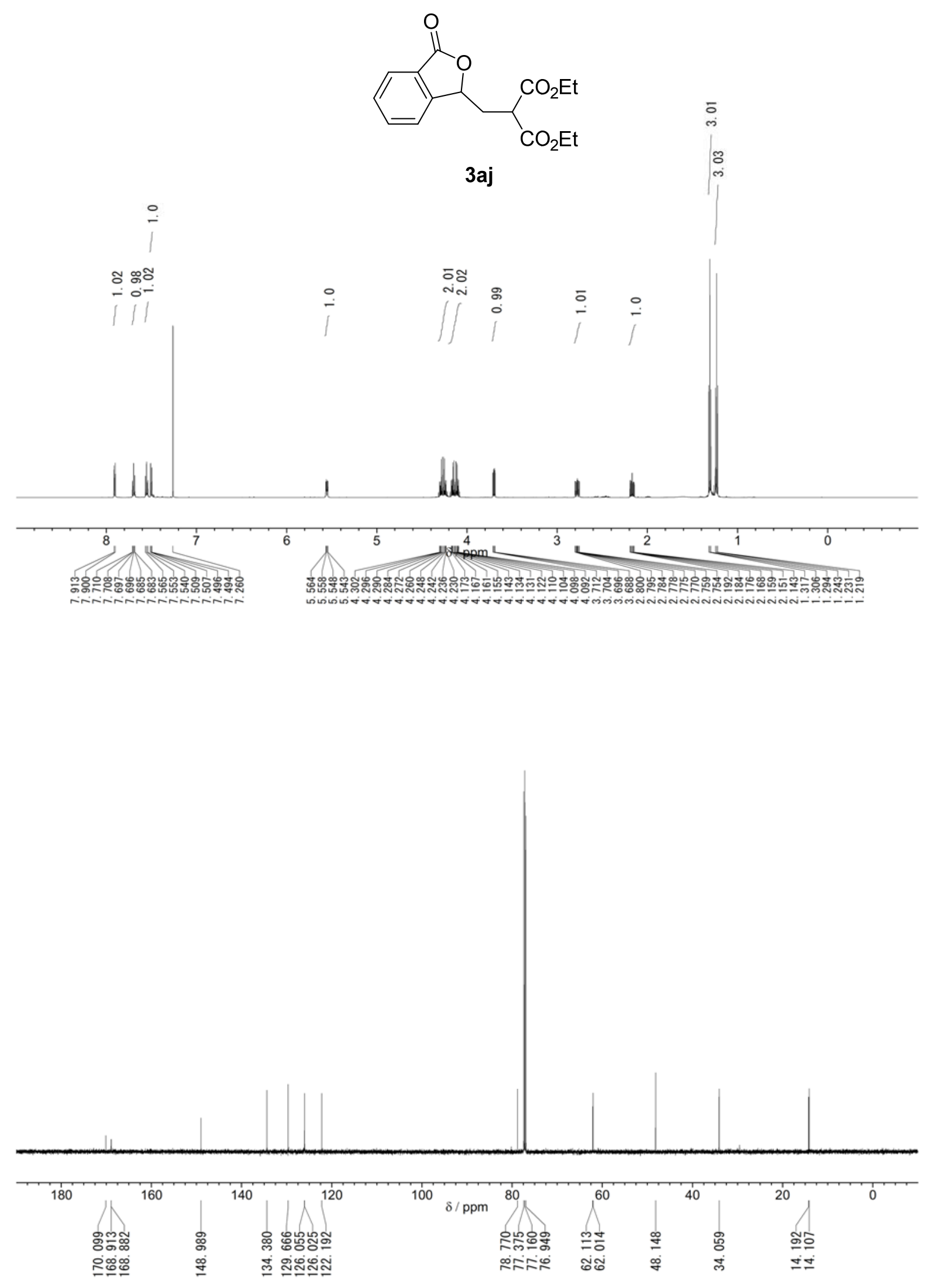

${ }^{1} \mathrm{H}$ NMR $(600 \mathrm{MHz})$ and ${ }^{13} \mathrm{C}\left\{{ }^{1} \mathrm{H}\right\}$ NMR $(150 \mathrm{MHz})$ spectra of 3aj $\left(\mathrm{CDCl}_{3}, \mathrm{rt}\right)$. 

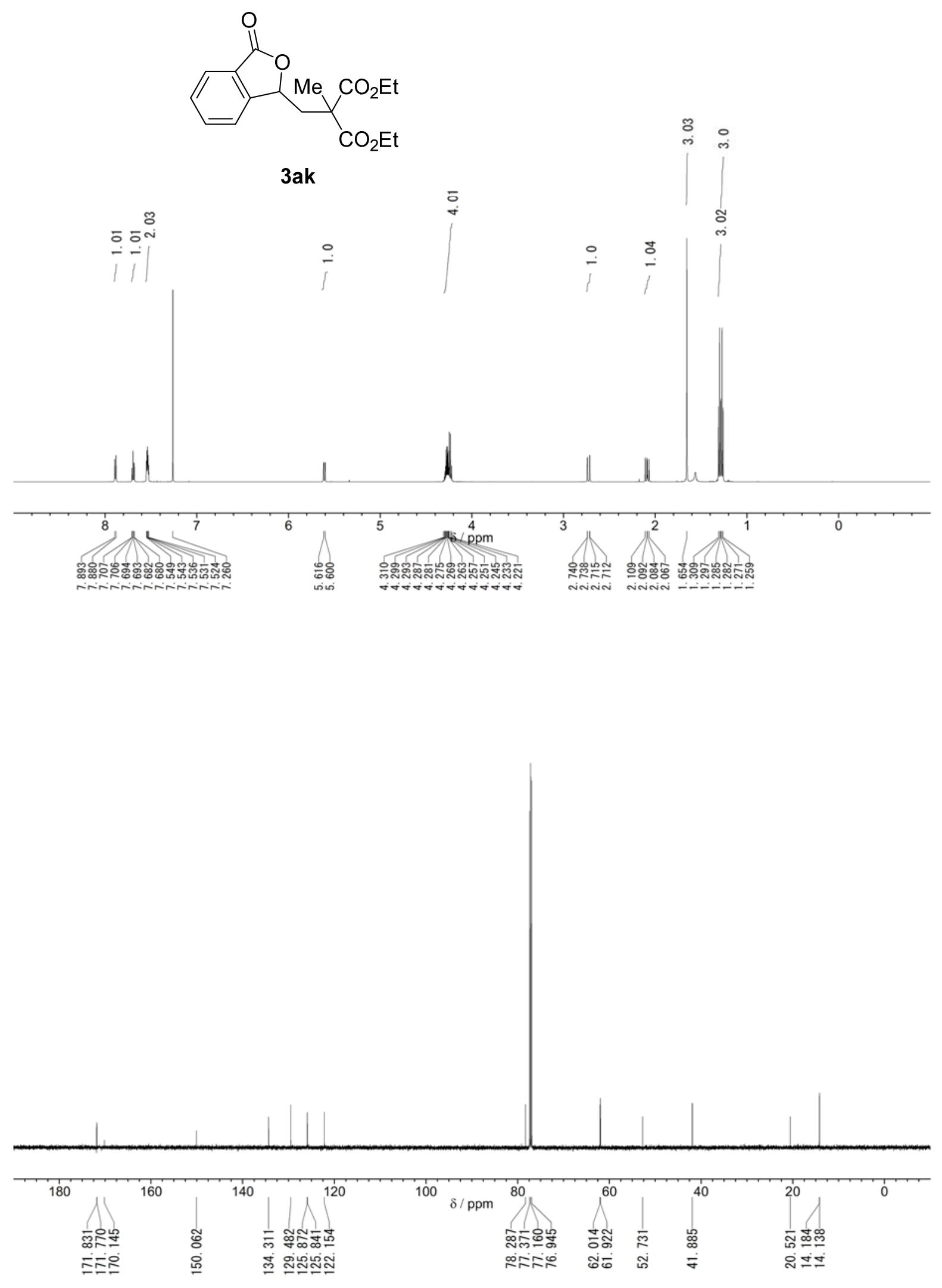

${ }^{1} \mathrm{H}$ NMR $(600 \mathrm{MHz})$ and ${ }^{13} \mathrm{C}\left\{{ }^{1} \mathrm{H}\right\}$ NMR $(150 \mathrm{MHz})$ spectra of 3ak $\left(\mathrm{CDCl}_{3}, \mathrm{rt}\right)$. 

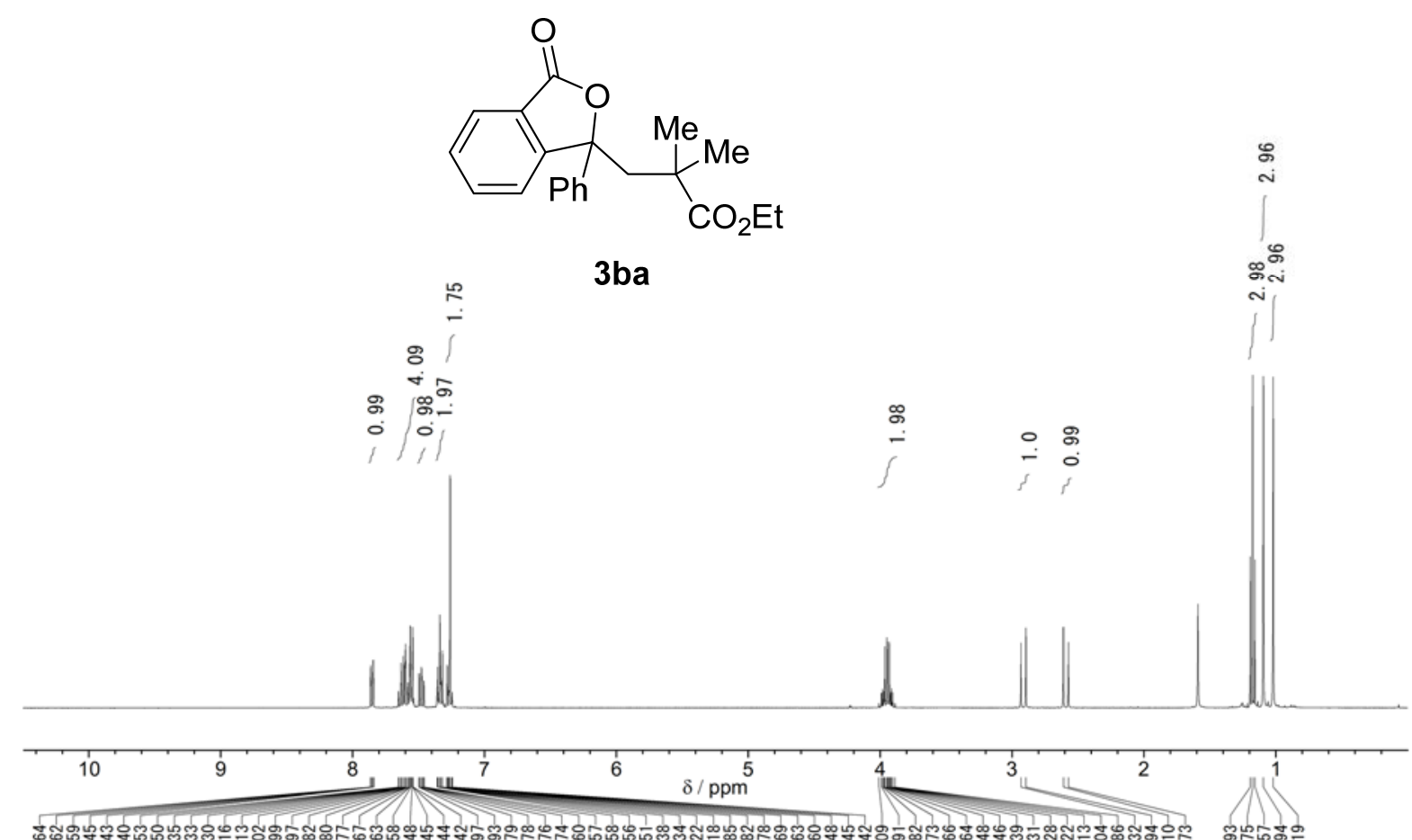

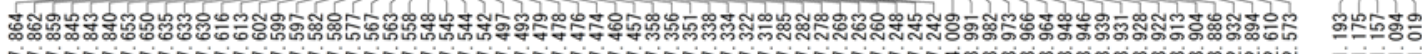

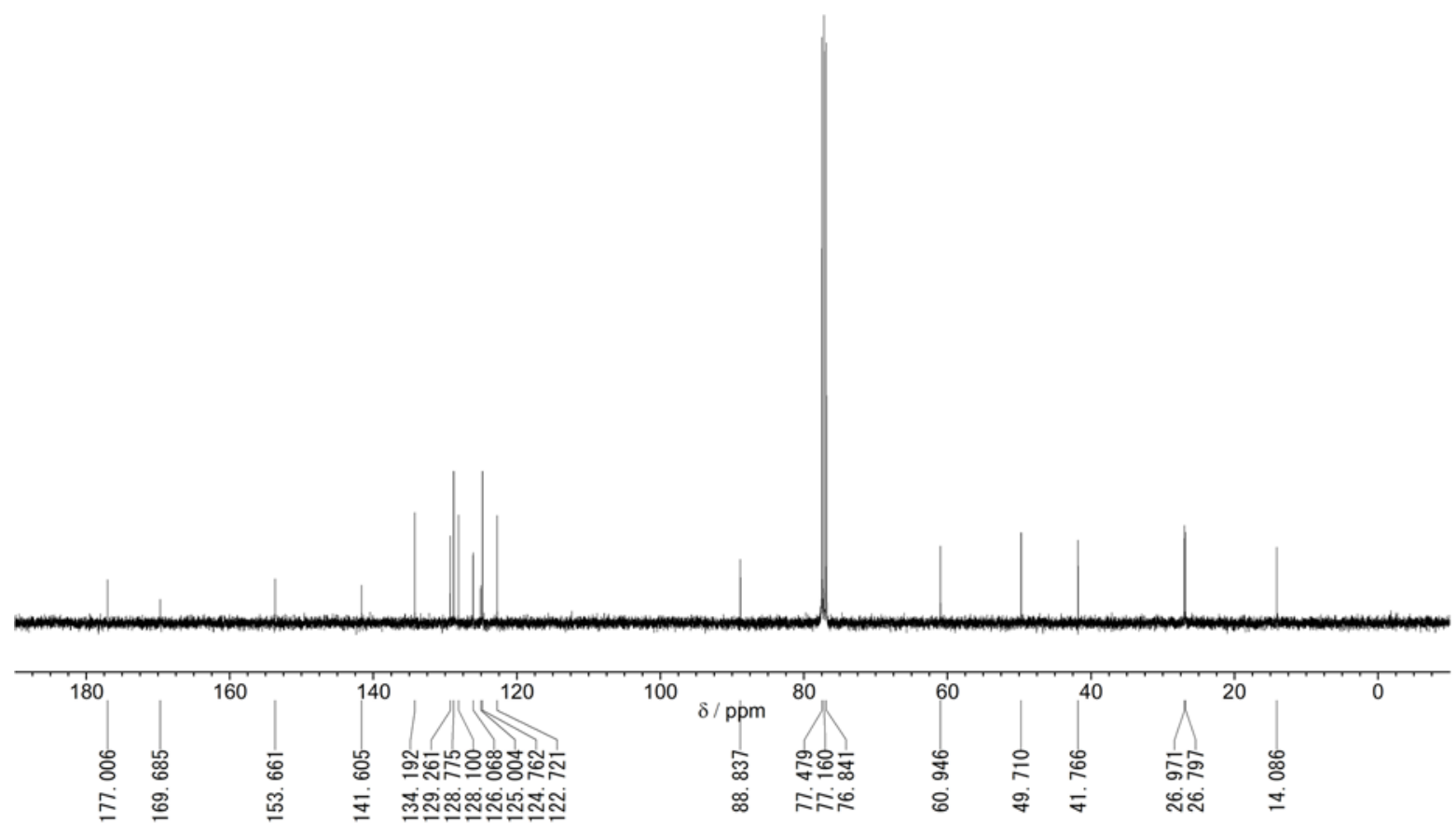

${ }^{1} \mathrm{H}$ NMR $(400 \mathrm{MHz})$ and ${ }^{13} \mathrm{C}\left\{{ }^{1} \mathrm{H}\right\}$ NMR $(100 \mathrm{MHz})$ spectra of $3 \mathbf{b a}\left(\mathrm{CDCl}_{3}, \mathrm{rt}\right)$. 

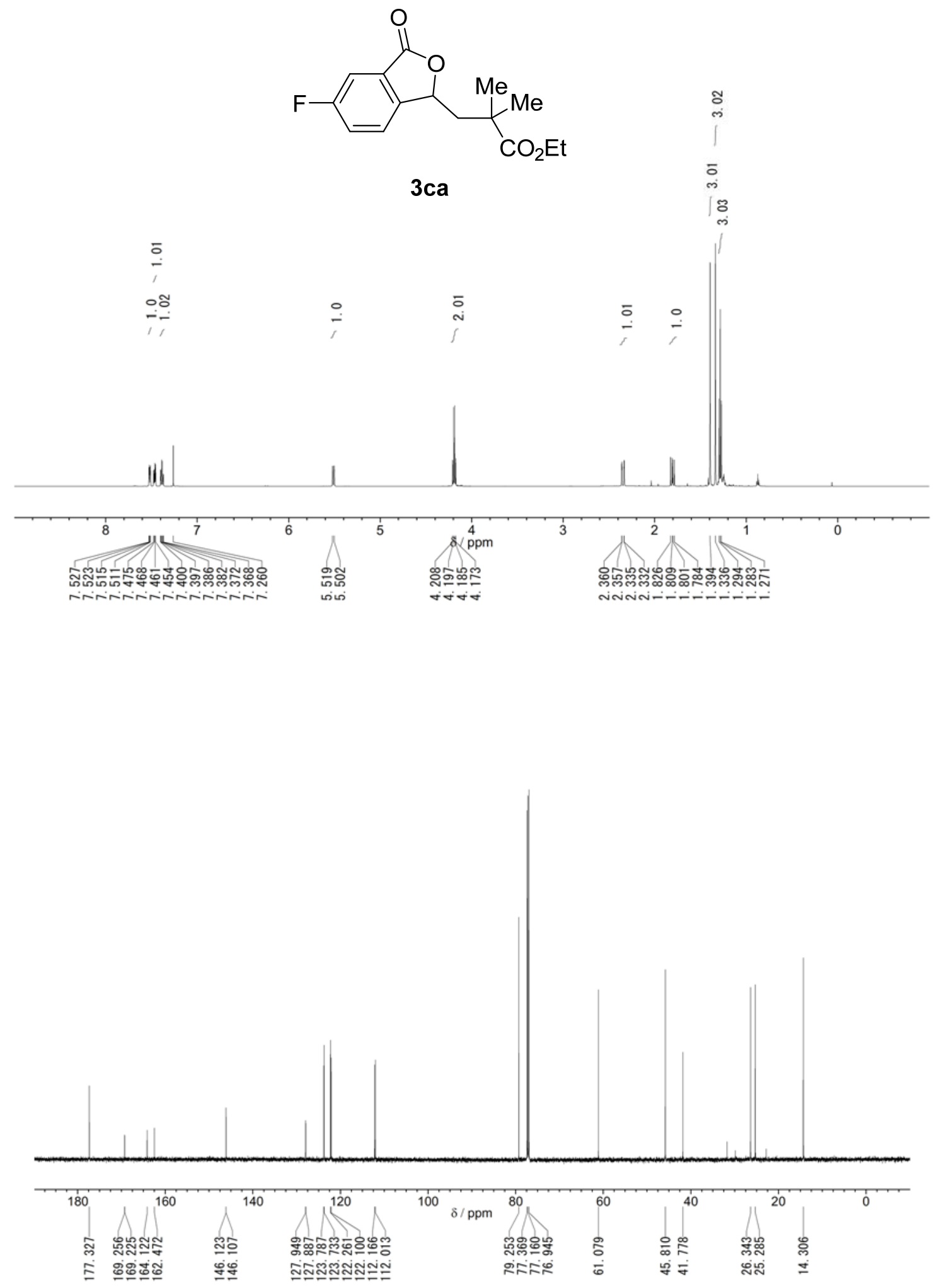

${ }^{1} \mathrm{H}$ NMR $(600 \mathrm{MHz})$ and ${ }^{13} \mathrm{C}\left\{{ }^{1} \mathrm{H}\right\}$ NMR $(150 \mathrm{MHz})$ spectra of 3ca $\left(\mathrm{CDCl}_{3}, \mathrm{rt}\right)$. 


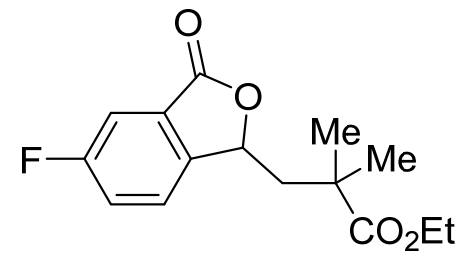

$3 c a$

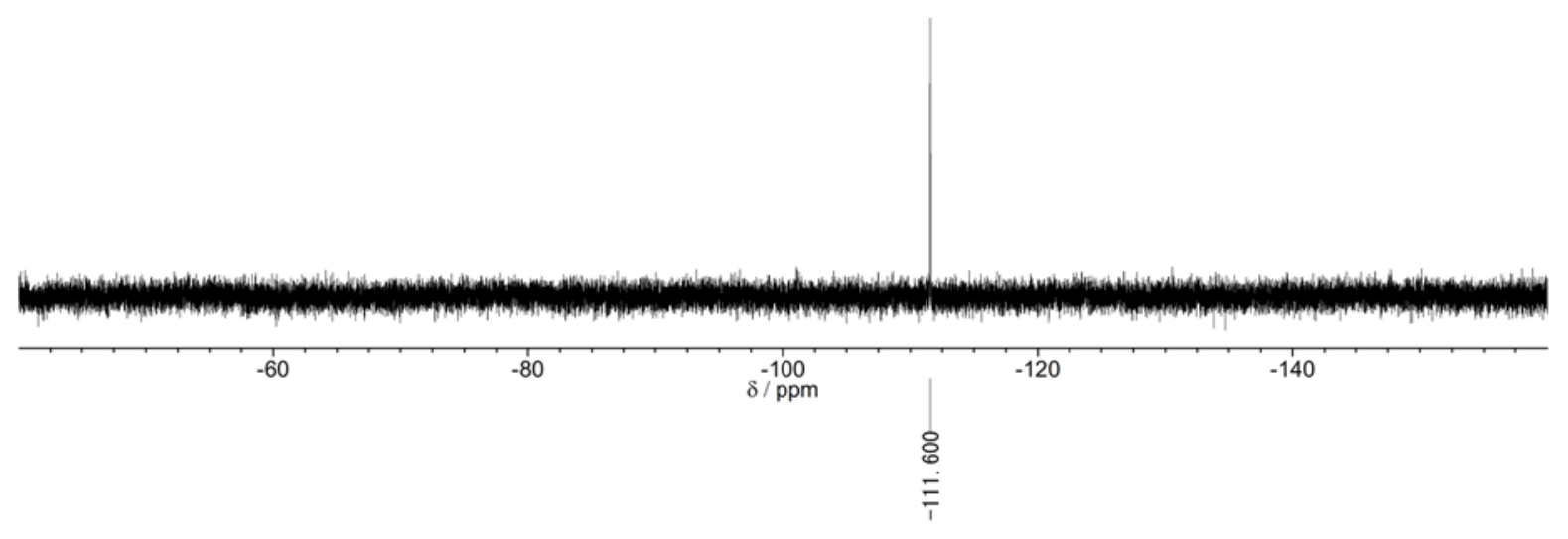

${ }^{19} \mathrm{~F}\left\{{ }^{1} \mathrm{H}\right\}$ NMR (564 MHz) spectra of 3ca $\left(\mathrm{CDCl}_{3}, \mathrm{rt}\right)$. 

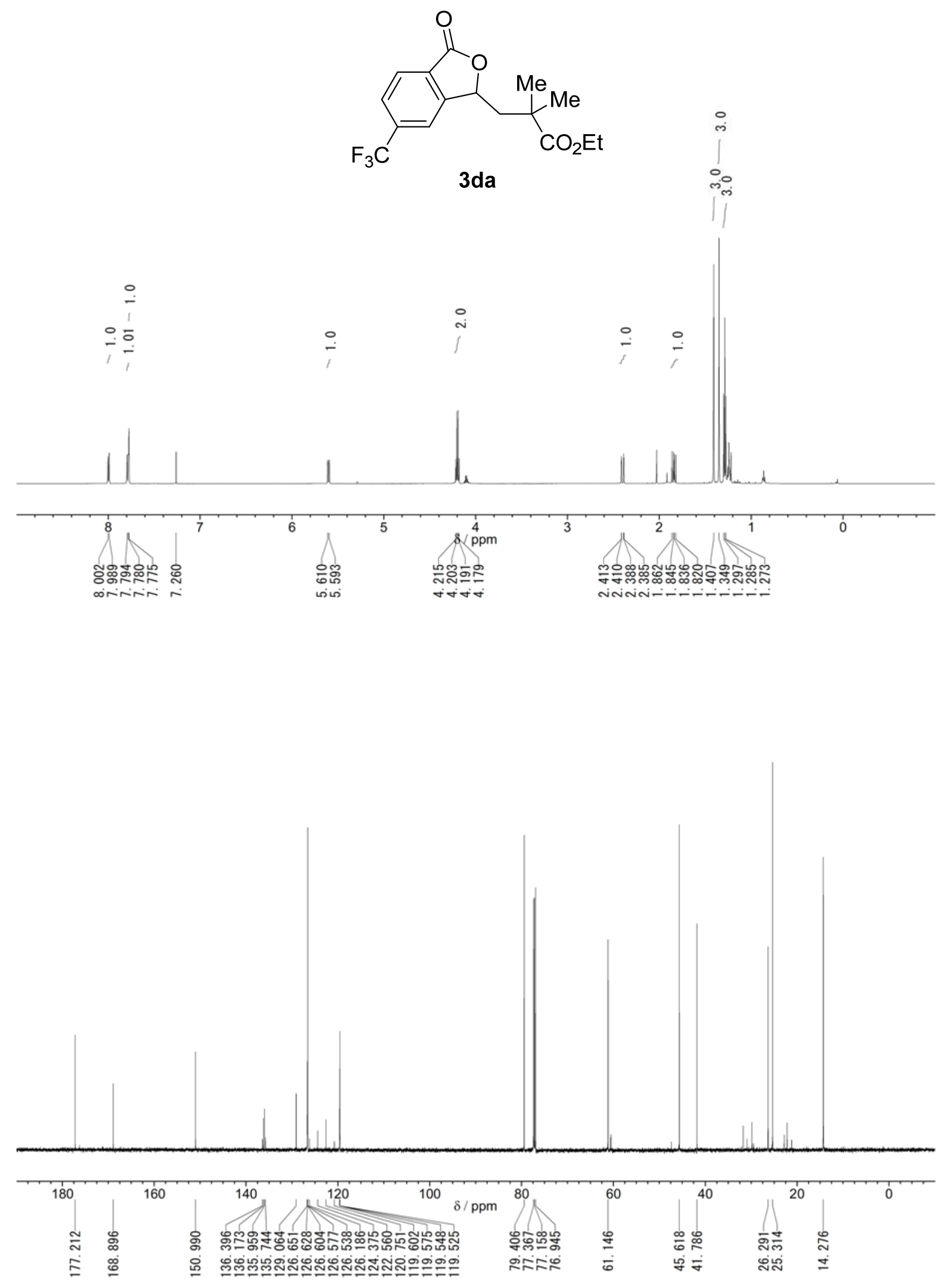

${ }^{1} \mathrm{H}$ NMR $(600 \mathrm{MHz})$ and ${ }^{13} \mathrm{C}\left\{{ }^{1} \mathrm{H}\right\}$ NMR $(150 \mathrm{MHz})$ spectra of 3da $\left(\mathrm{CDCl}_{3}, \mathrm{rt}\right)$. 


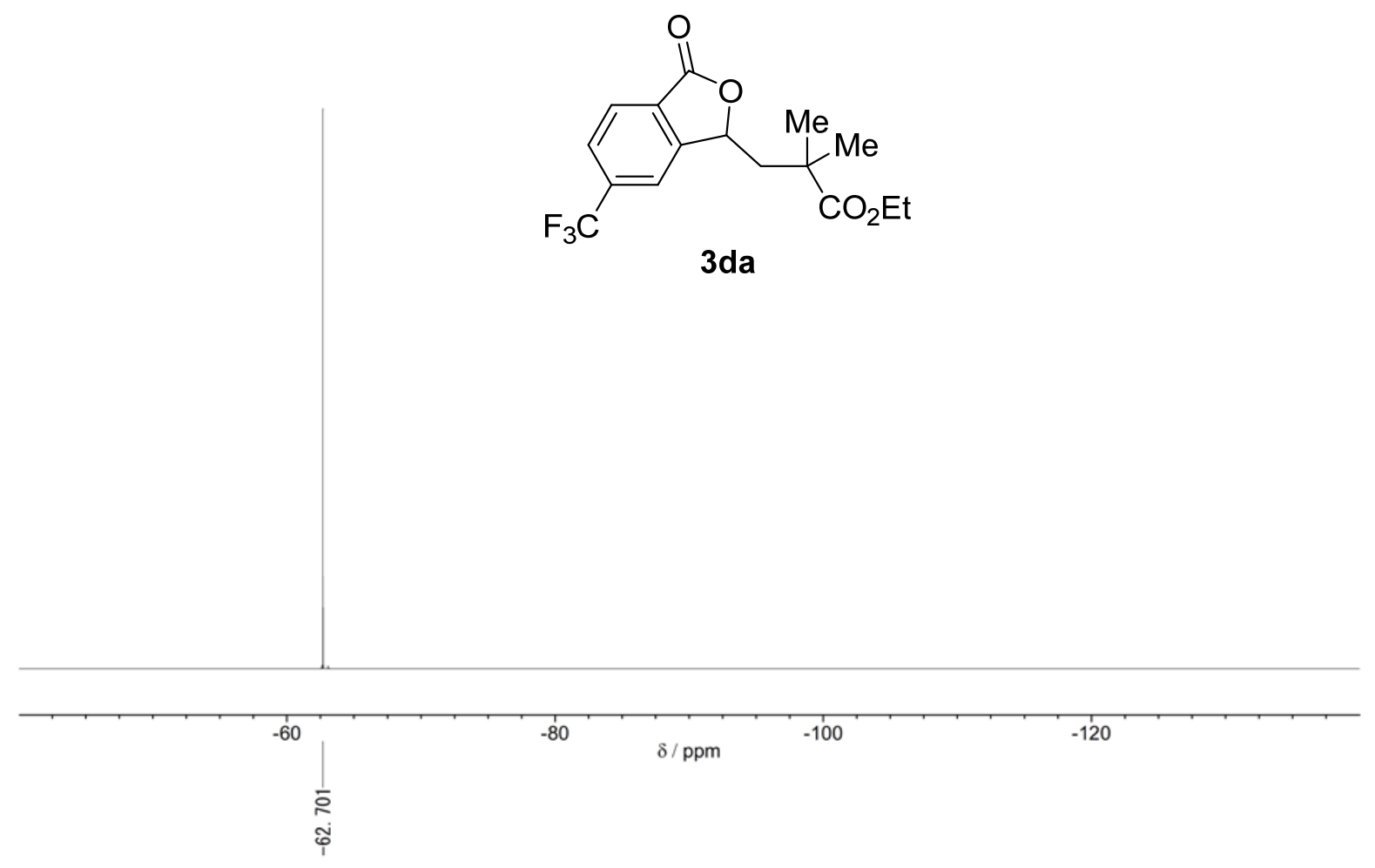

${ }^{19} \mathrm{~F}\left\{{ }^{1} \mathrm{H}\right\}$ NMR (564 MHz) spectra of 3da $\left(\mathrm{CDCl}_{3}, \mathrm{rt}\right)$. 

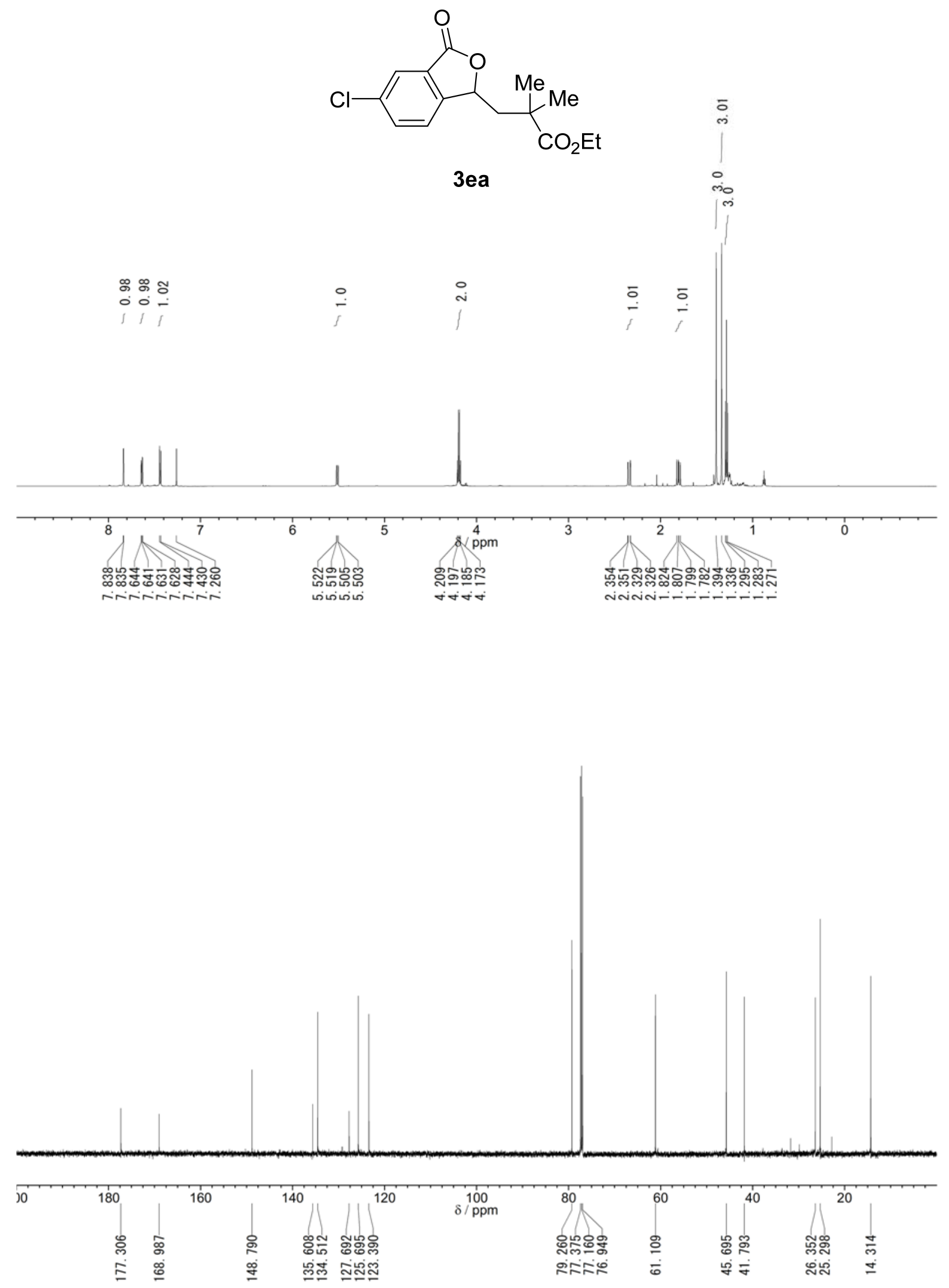

${ }^{1} \mathrm{H}$ NMR $(600 \mathrm{MHz})$ and ${ }^{13} \mathrm{C}\left\{{ }^{1} \mathrm{H}\right\}$ NMR $(150 \mathrm{MHz})$ spectra of 3ea $\left(\mathrm{CDCl}_{3}, \mathrm{rt}\right)$. 

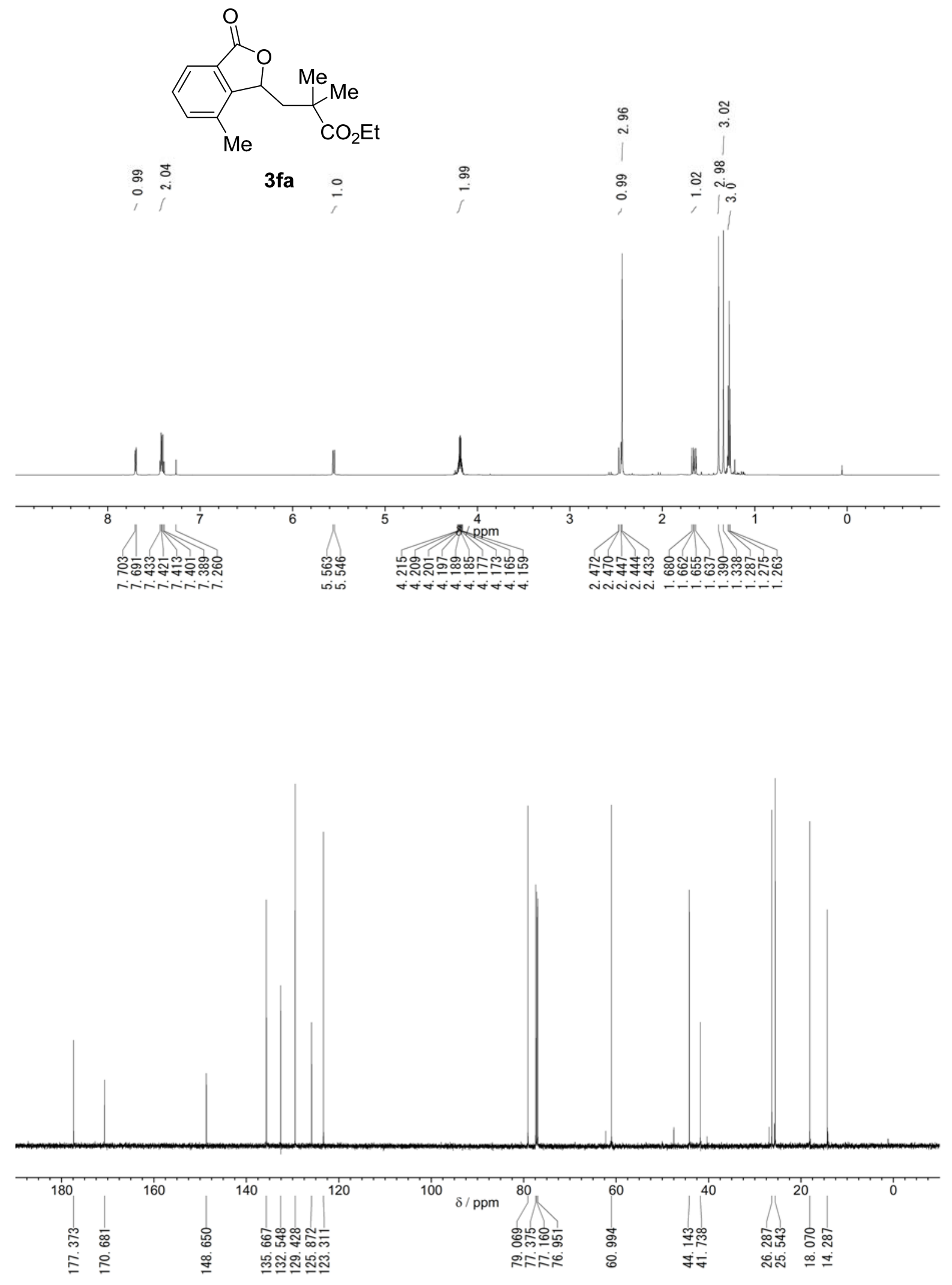

${ }^{1} \mathrm{H}$ NMR $(600 \mathrm{MHz})$ and ${ }^{13} \mathrm{C}\left\{{ }^{1} \mathrm{H}\right\}$ NMR $(150 \mathrm{MHz})$ spectra of $\mathbf{3 f a}\left(\mathrm{CDCl}_{3}, \mathrm{rt}\right)$. 

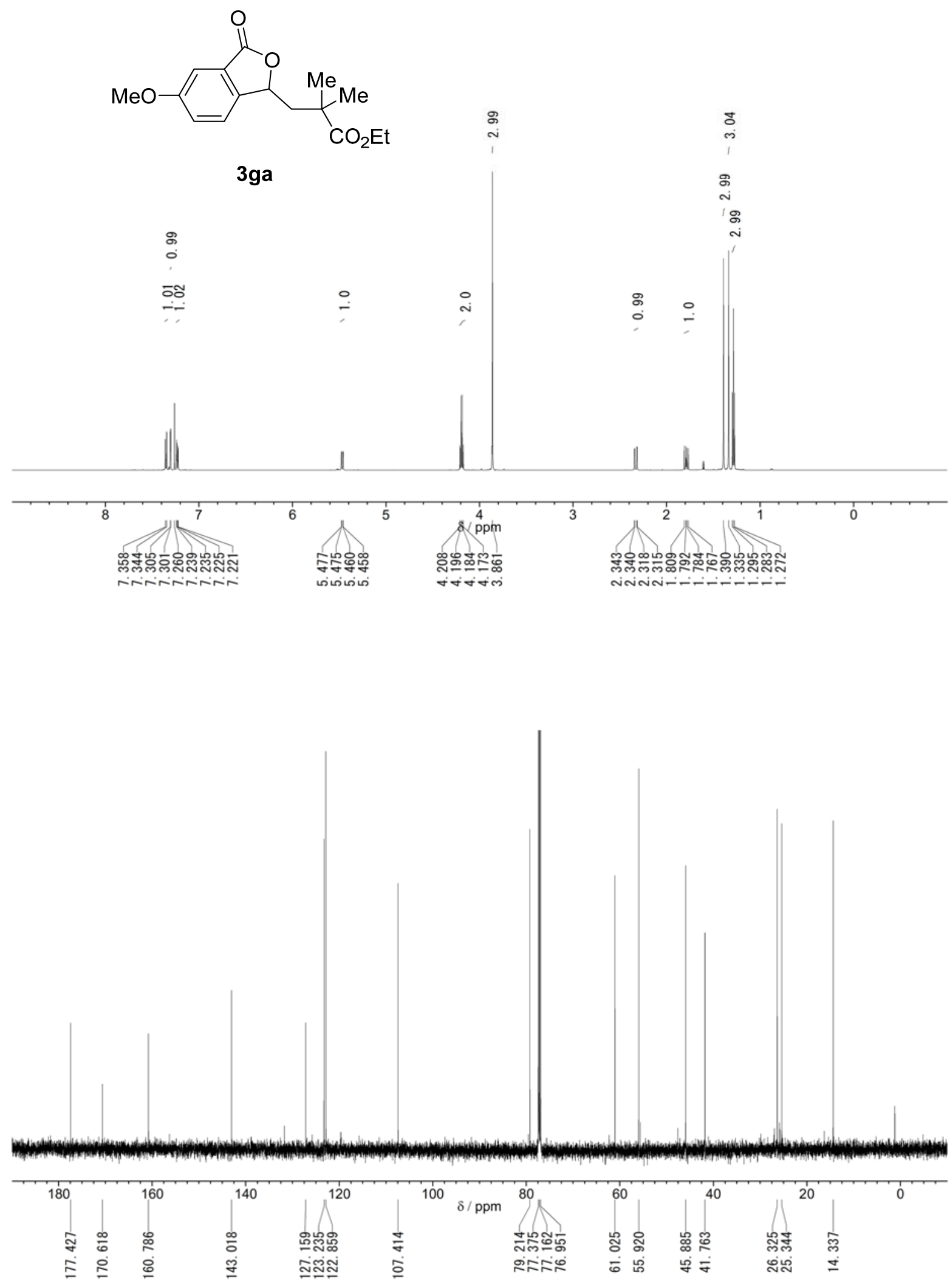

${ }^{1} \mathrm{H}$ NMR $(600 \mathrm{MHz})$ and ${ }^{13} \mathrm{C}\left\{{ }^{1} \mathrm{H}\right\}$ NMR $(150 \mathrm{MHz})$ spectra of $3 g a\left(\mathrm{CDCl}_{3}, \mathrm{rt}\right)$. 

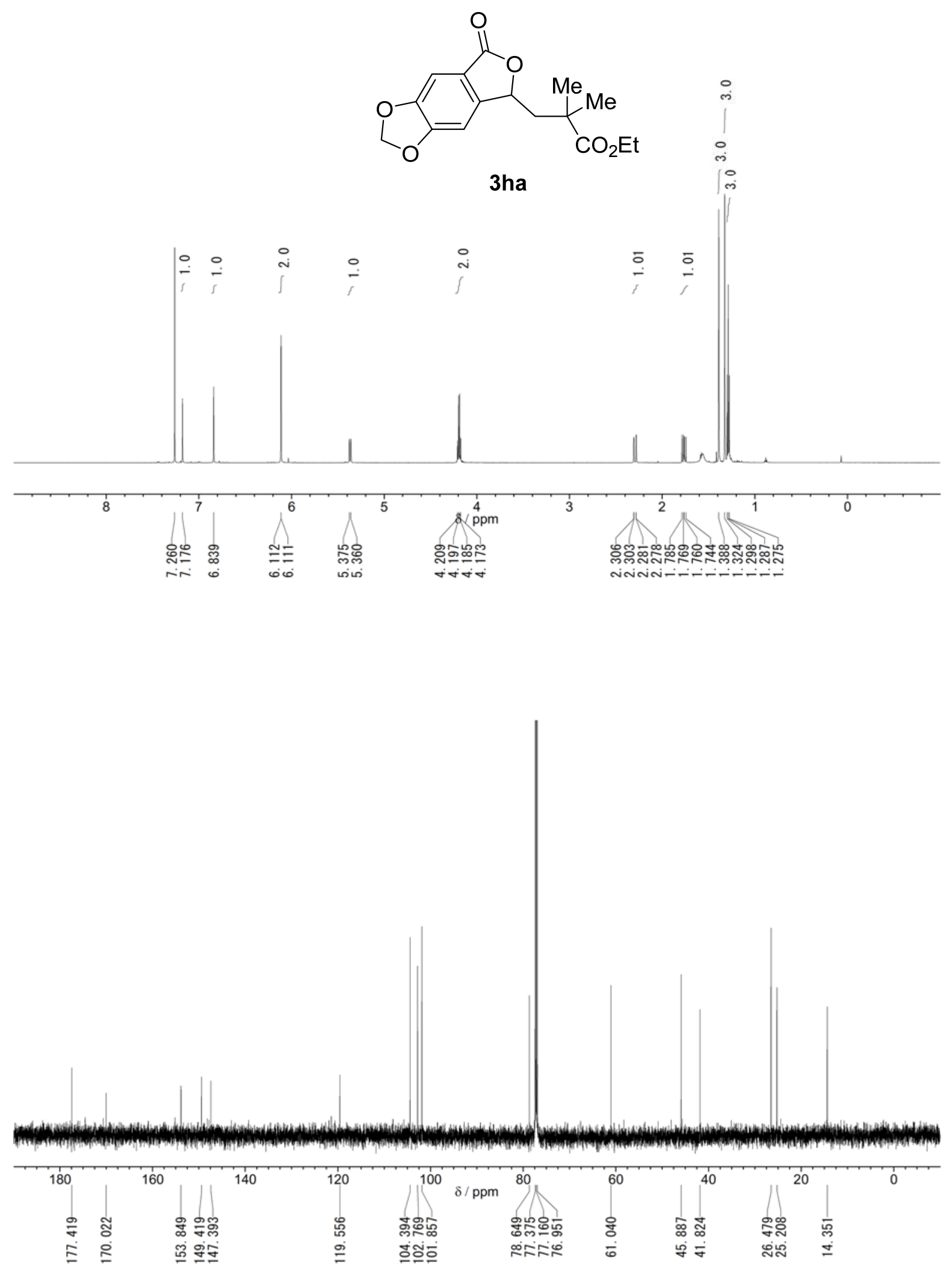

${ }^{1} \mathrm{H}$ NMR $(600 \mathrm{MHz})$ and ${ }^{13} \mathrm{C}\left\{{ }^{1} \mathrm{H}\right\}$ NMR $(150 \mathrm{MHz})$ spectra of 3ha $\left(\mathrm{CDCl}_{3}, \mathrm{rt}\right)$. 

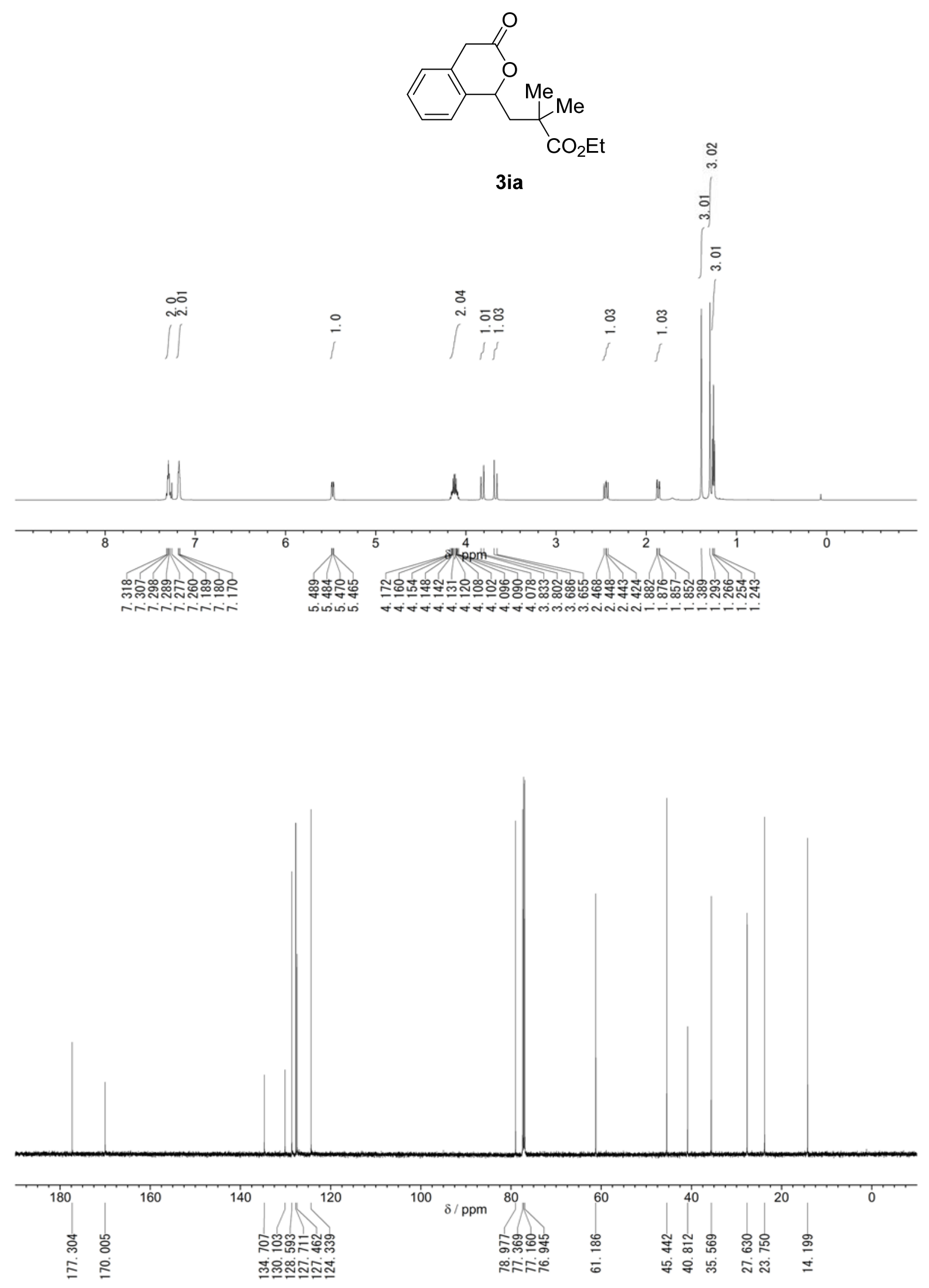

${ }^{1} \mathrm{H}$ NMR $(600 \mathrm{MHz})$ and ${ }^{13} \mathrm{C}\left\{{ }^{1} \mathrm{H}\right\}$ NMR $(150 \mathrm{MHz})$ spectra of 3ia $\left(\mathrm{CDCl}_{3}, \mathrm{rt}\right)$. 

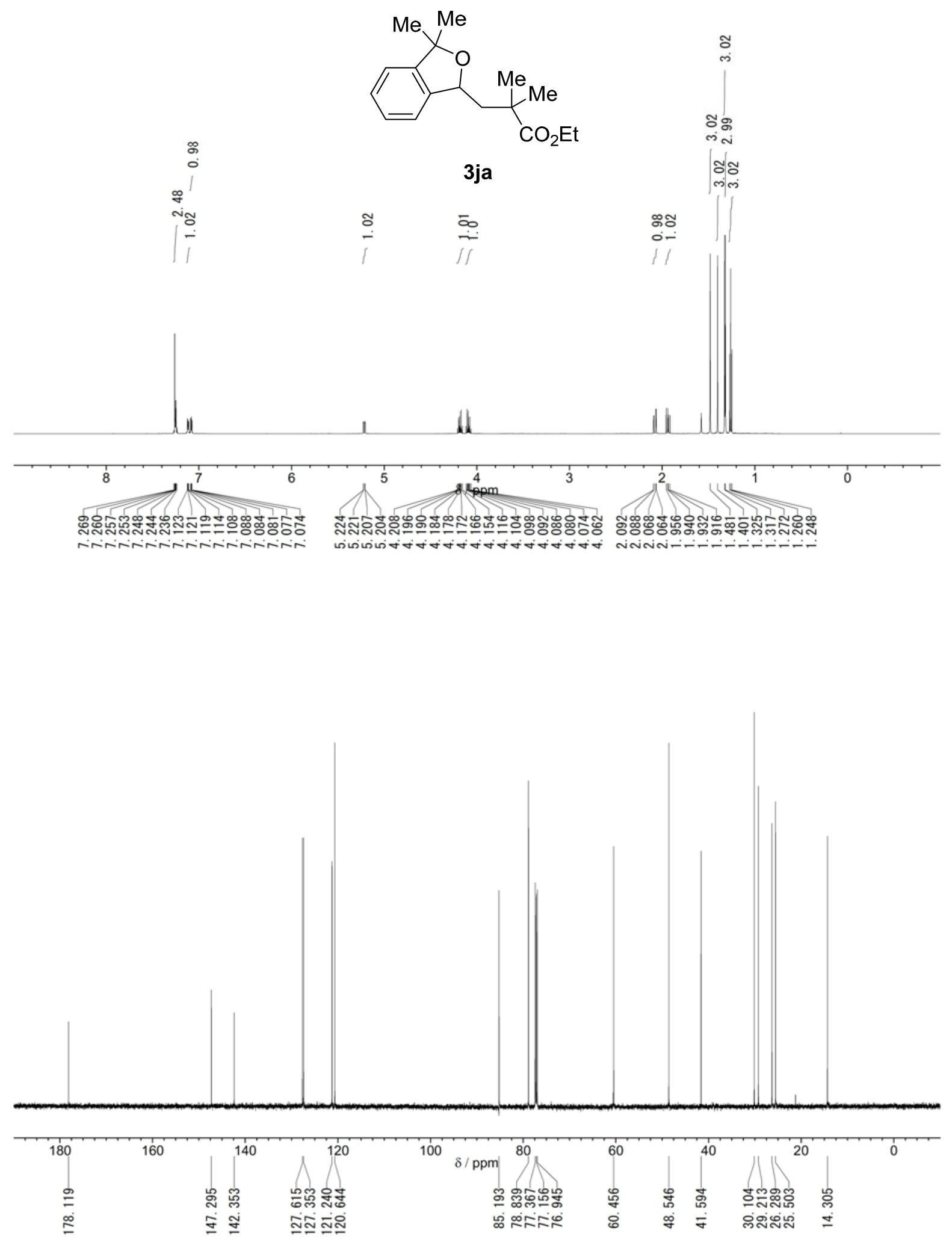

${ }^{1} \mathrm{H}$ NMR $(600 \mathrm{MHz})$ and ${ }^{13} \mathrm{C}\left\{{ }^{1} \mathrm{H}\right\}$ NMR $(150 \mathrm{MHz})$ spectra of $\mathbf{3 j a}\left(\mathrm{CDCl}_{3}, \mathrm{rt}\right)$. 

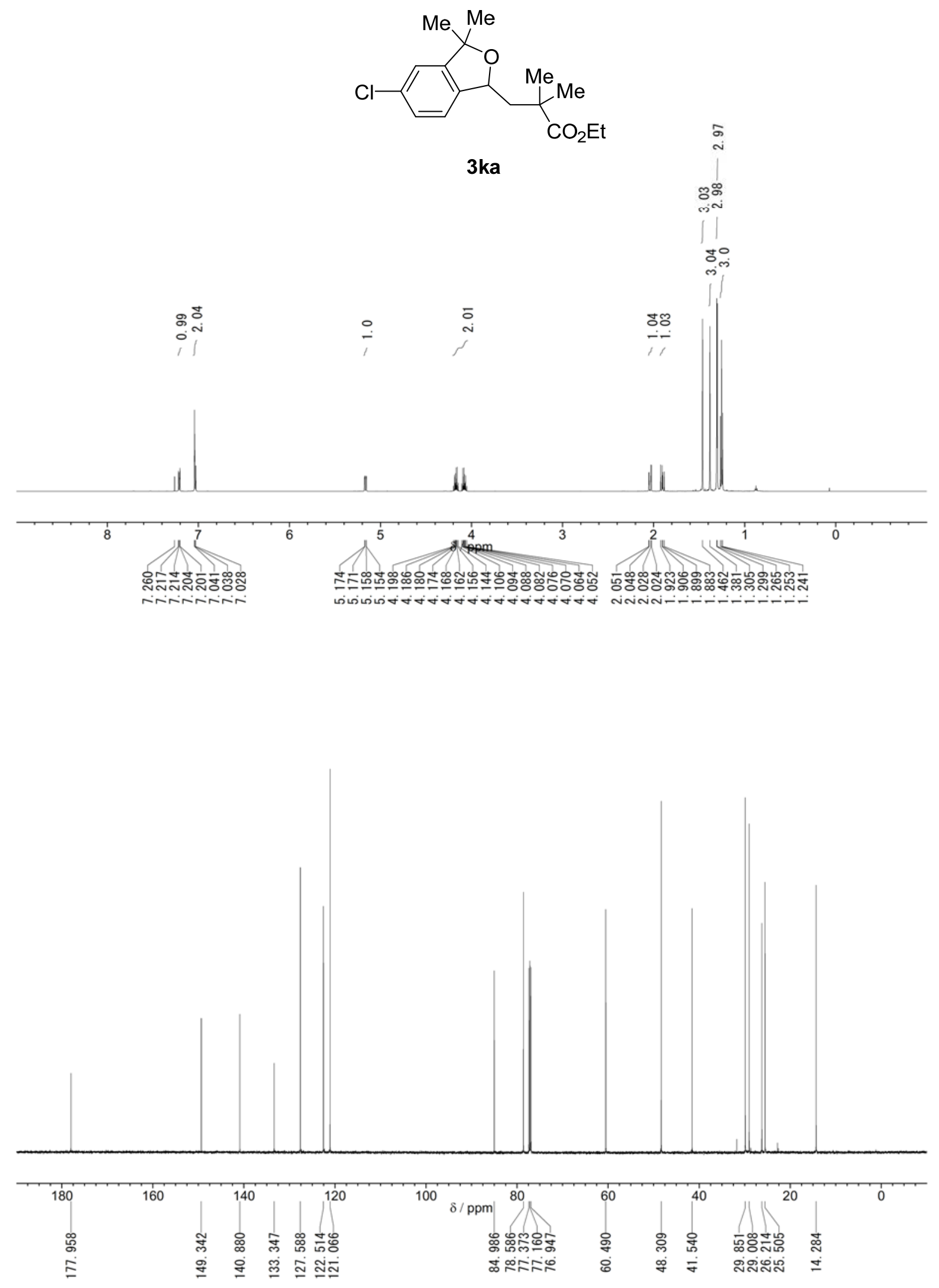

${ }^{1} \mathrm{H}$ NMR $(600 \mathrm{MHz})$ and ${ }^{13} \mathrm{C}\left\{{ }^{1} \mathrm{H}\right\}$ NMR $(150 \mathrm{MHz})$ spectra of 3ka $\left(\mathrm{CDCl}_{3}, \mathrm{rt}\right)$. 


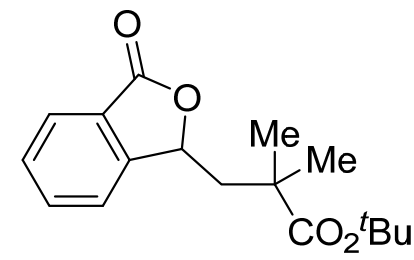

3al
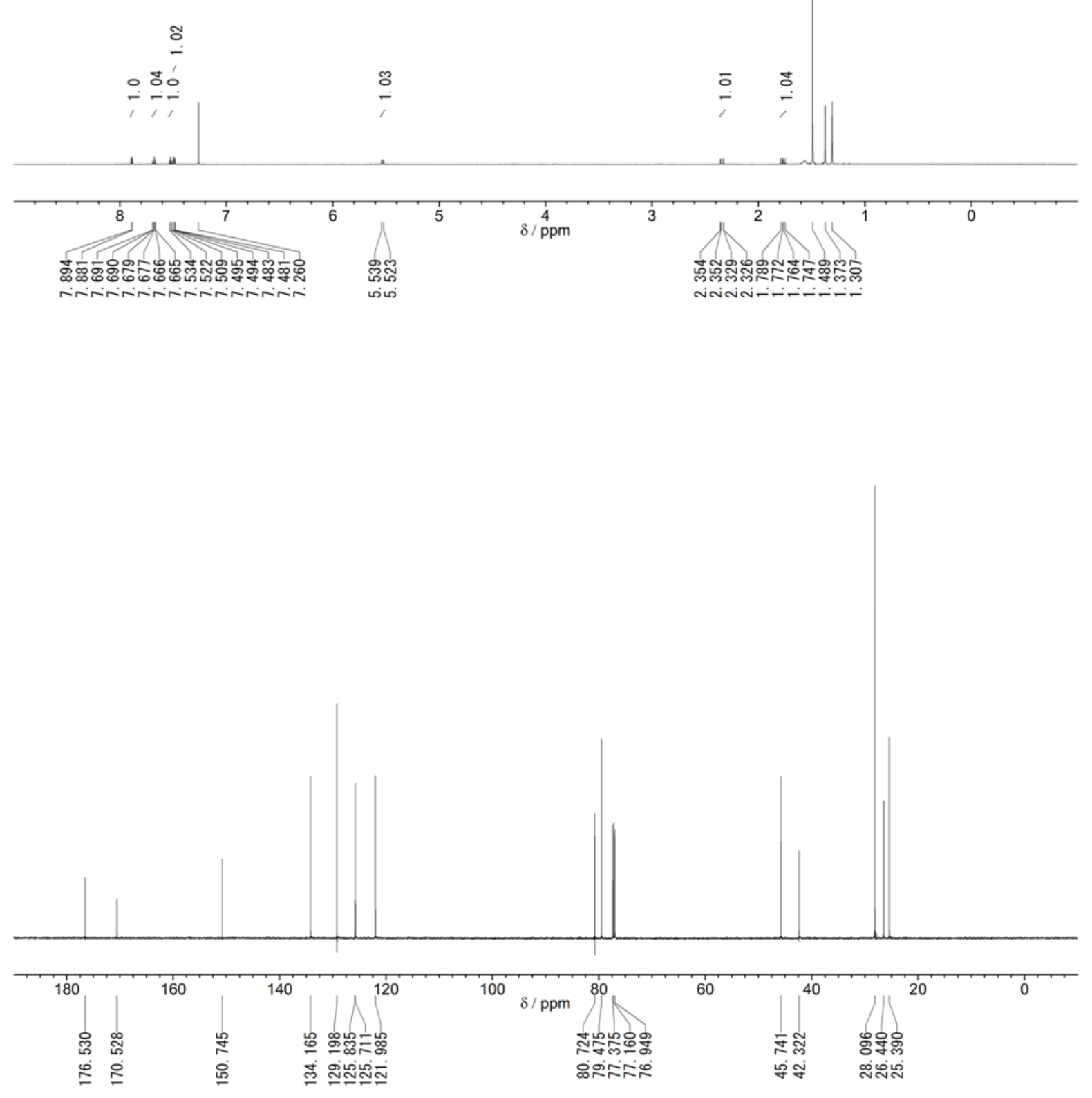

${ }^{1} \mathrm{H}$ NMR $(600 \mathrm{MHz})$ and ${ }^{13} \mathrm{C}\left\{{ }^{1} \mathrm{H}\right\}$ NMR $(150 \mathrm{MHz})$ spectra of 3 al $\left(\mathrm{CDCl}_{3}, \mathrm{rt}\right)$. 

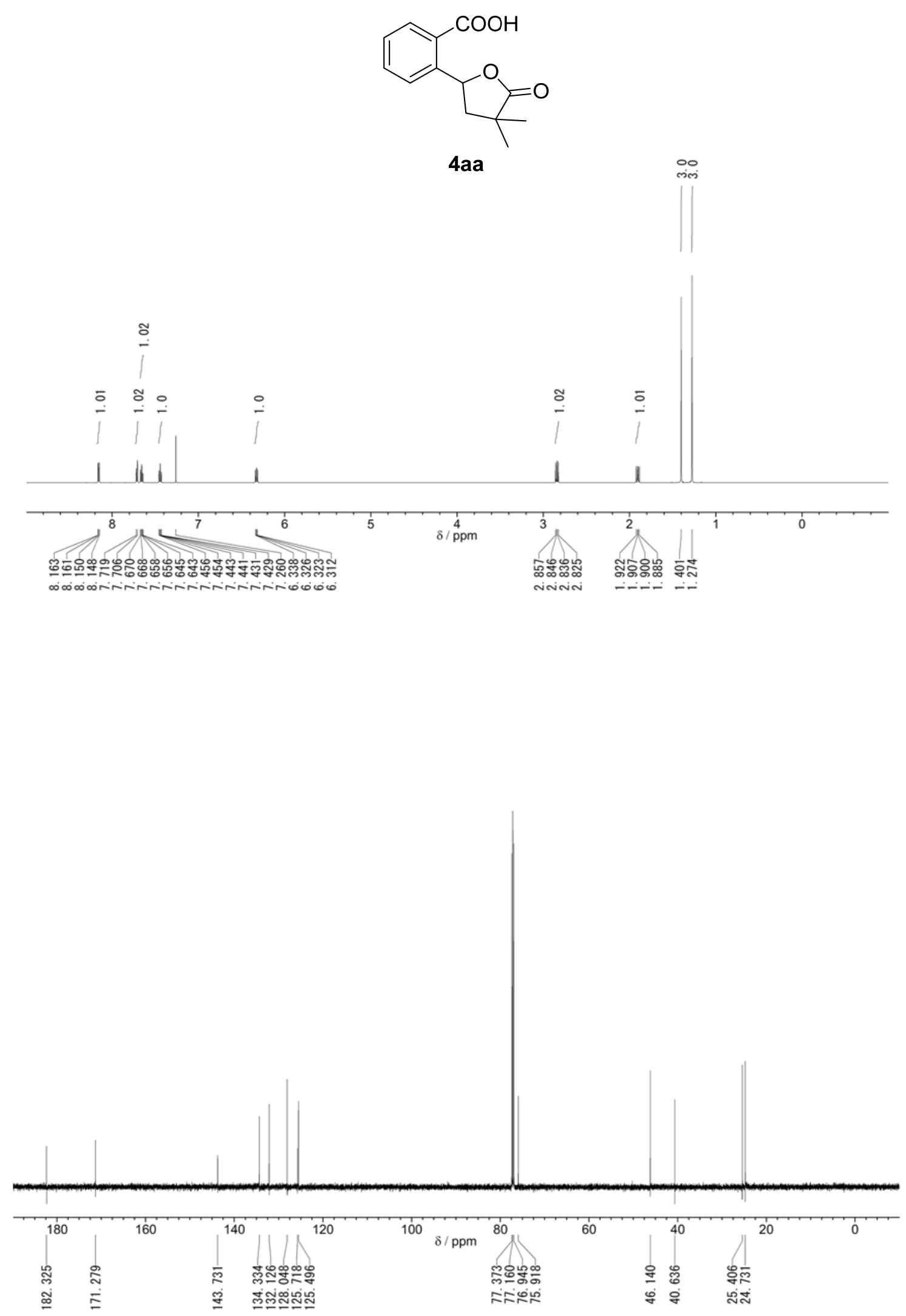

${ }^{1} \mathrm{H}$ NMR $(600 \mathrm{MHz})$ and ${ }^{13} \mathrm{C}\left\{{ }^{1} \mathrm{H}\right\}$ NMR $(150 \mathrm{MHz})$ spectra of 4aa $\left(\mathrm{CDCl}_{3}, \mathrm{rt}\right)$. 


\section{References}

(1) (a) Dydio, P.; Reek, J. N. H. Supramolecular Control of Selectivity in Hydroformylation of Vinyl Arenes: Easy Access to Valuable $\beta$-Aldehyde Intermediates. Angew. Chem. Int. Ed. 2013, 52, 3878-3882. (b) Sha, W.; Zhang, W.; Ni, S.; Mei, H.; Han, J; Pan Y. Photoredox-Catalyzed Cascade Difluoroalkylation and Intramolecular Cyclization for Construction of Fluorinated $\gamma$-Butyrolactones. J. Org. Chem. 2017, 82, 9824-9831. (c) Chen, D.; Chemler, S. R. Synthesis of Phthalans Via Copper-Catalyzed Enatioselective Cyclization/Carboetherfication of 2-Vinylbenzyl Alcohols. Org. Lett. 2018, 20, 6453-6456. (d) Gaenzler, F. C; Guo, C.; Zhang, Y.-W.; Azab, M. E.; Salem, M. A. I.; Fan, D. P.; Smith, M. B. 2Dienylphenacyloxazolones and an intramolecular Diels-Alder approach to the A-B-C ring system of phenanthridone alkaloids. Tetrahedron 2009, 65, 8781-8785.

(2) (a) Epstein, J. W.; Brabander, H. J.; Fanshawe, W. J.; Hofmann, C. M.; McKenzie, T. C.; Safir, S. R.; Osterberg, A. C.; Cosulich, D. B.; Lovell, F. M. 1-Aryl-3-azabicyclo[3.1.0]hexanes, a New Series of Nonnarcotic Analgesic Agents. J. Med. Chem. 1981, 24, 481-490. (b) Ishihara, J.; Watanabe, Y.; Koyama, N.; Nishino, Y.; Takahashi, K.; Hatakeyama, S. Indium-mediated Reformatsky-Claisen rearrangement. Tetrahedron 2011, 67, 3659-3667. (c) Wang, Y.; Shao, Z.; Zhang, K.; Liu, Q. Manganese-Catalyzed Dual-Deoxygenative Coupling of Primary Alcohols with 2-Arylethanols. Angew. Chem. Int. Ed. 2018, $57,15143-15147$. 